\title{
PROPER MOTIONS OF THE ARCHES CLUSTER WITH KECK LASER GUIDE STAR ADAPTIVE OPTICS: THE FIRST KINEMATIC MASS MEASUREMENT OF THE ARCHES
}

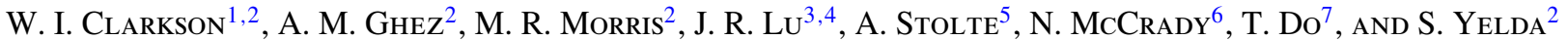 \\ ${ }^{1}$ Division of Astronomy and Astrophysics, University of California, Los Angeles, Physics and Astronomy Building, \\ 430 Portola Plaza, Box 951547, Los Angeles, CA 90095-1547, USA \\ ${ }^{2}$ Department of Astronomy, Indiana University, Bloomington, 727 East 3rd Street, Swain West 319, Bloomington, IN 47405-7105, USA; wiclarks@indiana.edu \\ ${ }^{3}$ Institute for Astronomy, 2680 Woodlawn Drive, Honolulu, HI 96822-1839, USA \\ ${ }^{4}$ Department of Astronomy, California Institute of Technology, 1200 East California Boulevard, Pasadena, CA 91125, USA \\ ${ }^{5}$ Argelander Institut für Astronomie, Universität Bonn, Auf dem Hügel 71, 53121 Bonn, Germany \\ ${ }^{6}$ Department of Physics and Astronomy, University of Montana, 32 Campus Drive, No. 1080, Missoula, MT 59812, USA \\ ${ }^{7}$ Department of Physics and Astronomy, University of California, Irvine, 4129 Frederick Reines Hall, Irvine, CA 92697-4575, USA \\ Received 2011 September 27; accepted 2011 December 14; published 2012 May 15
}

\begin{abstract}
We report the first detection of the intrinsic velocity dispersion of the Arches cluster-a young ( $2 \mathrm{Myr}$ ), massive $\left(10^{4} M_{\odot}\right)$ starburst cluster located only $26 \mathrm{pc}$ in projection from the Galactic center. This was accomplished using proper motion measurements within the central $10^{\prime \prime} \times 10^{\prime \prime}$ of the cluster, obtained with the laser guide star adaptive optics system at Keck Observatory over a three-year time baseline (2006-2009). This uniform data set results in proper motion measurements that are improved by a factor $\sim 5$ over previous measurements from heterogeneous instruments. By careful, simultaneous accounting of the cluster and field contaminant distributions as well as the possible sources of measurement uncertainties, we estimate the internal velocity dispersion to be $0.15 \pm 0.01 \mathrm{mas} \mathrm{yr}^{-1}$, which corresponds to $5.4 \pm 0.4 \mathrm{~km} \mathrm{~s}^{-1}$ at a distance of $8.4 \mathrm{kpc}$. Projecting a simple model for the cluster onto the sky to compare with our proper motion data set, in conjunction with surface density data, we estimate the total present-day mass of the cluster to be $M(r<1.0 \mathrm{pc})=1.5_{-0.60}^{+0.74} \times 10^{4} M_{\odot}$. The mass in stars observed within a cylinder of radius $R$ (for comparison to photometric estimates) is found to be $M(R<0.4 \mathrm{pc})=0.90_{-0.35}^{+0.40} \times 10^{4} M_{\odot}$ at formal $3 \sigma$ confidence. This mass measurement is free from assumptions about the mass function of the cluster, and thus may be used to check mass estimates from photometry and simulation. Photometric mass estimates assuming an initially Salpeter mass function $\left(\Gamma_{0}=1.35\right.$, or $\Gamma \sim 1.0$ at present, where $\left.d N / d(\log M) \propto M^{\Gamma}\right)$ suggest a total cluster mass $M_{\mathrm{cl}} \sim(4-6) \times 10^{4} M_{\odot}$ and projected mass $(\sim 2 \leqslant M(R<0.4 \mathrm{pc}) \leqslant 3) \times 10^{4} M_{\odot}$. Photometric mass estimates assuming a globally top-heavy or strongly truncated present-day mass function (PDMF; with $\Gamma \sim 0.6$ ) yield mass estimates closer to $M(R<0.4 \mathrm{pc}) \sim 1-1.2 \times 10^{4} M_{\odot}$. Consequently, our results support a PDMF that is either topheavy or truncated at low mass, or both. Collateral benefits of our data and analysis include: (1) cluster membership probabilities, which may be used to extract a clean-cluster sample for future photometric work; (2) a refined estimate of the bulk motion of the Arches cluster with respect to the field, which we find to be $172 \pm 15 \mathrm{~km} \mathrm{~s}^{-1}$, which is slightly slower than suggested by previous measurements using one epoch each with the Very Large Telescope and the Keck telescope; and (3) a velocity dispersion estimate for the field itself, which is likely dominated by the inner Galactic bulge and the nuclear disk.
\end{abstract}

Key words: astrometry - galaxies: clusters: individual (Arches) - Galaxy: kinematics and dynamics - open clusters and associations: individual (Arches) - stars: kinematics and dynamics - techniques: high angular resolution

Online-only material: color figures, machine-readable table

\section{INTRODUCTION}

The spectrum of masses produced during the star formation process (the initial mass function (IMF)) is a key prediction of the star formation process as it indirectly links to the observable present-day mass function (PDMF) of the population (for example, see Miller \& Scalo 1979; McKee \& Ostriker 2007; Bastian et al. 2010 for review). Because star formation depends on collapse by self-gravity out of a turbulent medium threaded with a magnetic field, there is some expectation that the physical conditions in the parent cloud should affect the slope of the IMF, its minimum mass, or both (e.g., Morris 1993). Numerical modeling provides some support for environment-dependent IMF variations, particularly in the unusual environment of the Galactic center (e.g., Bonnell et al. 2004; Klessen et al. 2007; Krumholz \& McKee 2008; Bonnell \& Rice 2008). However, the resulting IMF variations may be so small as to be observable only in extreme environments (e.g., Elmegreen et al. 2008). There is some observational support for a varying IMF and turnover mass in the extreme environments of the young $\left(\sim\right.$ few Myr), massive $\left(\sim 10^{4} M_{\odot}\right)$ starburst clusters NGC 3603 (Harayama et al. 2008) and for the stellar cluster at the Galactic center itself (Bartko et al. 2010).

The young, massive cluster known as the Arches cluster (e.g., Nagata et al. 1995; Cotera et al. 1996) ${ }^{8}$ is a particularly wellstudied example of an extreme environment for star formation. It is massive (total mass $M_{\mathrm{cl}} \sim(2-7) \times 10^{4} M_{\odot}$; Figer et al. 1999, $2002)$, dense $\left(\rho_{c} \sim 10^{5} M_{\odot} \mathrm{pc}^{-3}\right.$; Espinoza et al. 2009), and young ( 2-2.5 Myr; Najarro et al. 2004; Martins et al. 2008). It contains a substantial number of massive stars (Serabyn et al.

\footnotetext{
8 Throughout this paper, "the Arches" refers to the star cluster, not the arched radio filaments (Yusef-Zadeh et al. 1984; Morris \& Yusef-Zadeh 1989), against which the cluster is projected, and with which it is physically associated (Lang 2004).
} 
1998) which both contribute to and heat the surrounding medium (e.g., Figer et al. 2002; Yusef-Zadeh et al. 2002; Lang 2004).

The Arches cluster is located only $26 \mathrm{pc}$ in projection from the Galactic center (hereafter GC). It therefore likely formed in an environment characterized by high gas pressure and velocity dispersion in the parent cloud, and high ambient temperature, particularly when compared to the relatively more benign environment of NGC 3603. As these parameters are thought to impact the IMF (Morris 1993; Klessen et al. 2007), the Arches cluster is expected to be an excellent candidate for observing a non-canonical IMF, whether in its mass function exponent, low-mass turnover, a low-mass cutoff, or all three (e.g., Stolte et al. 2002; Klessen et al. 2007). It is also young enough that the most massive main-sequence stars are still present, making it one of the few clusters in which the upper mass limit to the star formation process may be observationally tested (Figer 2005; Crowther et al. 2010). It has thus received substantial observational attention, with efforts focused particularly on estimates of its IMF. Indeed, the Arches was originally the prototypical object for a non-standard IMF (Figer et al. 1999), with an observed present-day luminosity function indicating an overabundance of massive stars compared to the canonical Salpeter IMF (parameterized as $d N / d(\log M) \propto M^{-\Gamma}$, with $\Gamma=1.35$; see Bastian et al. 2010 for a review).

However, a number of effects conspire to obscure the true IMF from observation, complicating the interpretation of the PDMF, and indeed the present consensus seems to be that the Arches began with an IMF that is consistent with the canonical Salpeter IMF found in most environments. Photometric efforts to chart the present-day luminosity function of the cluster suffer from two important limitations. First, the observations are difficult; strong crowding and high, spatially variable extinction are observed across the field of view, so that the photometric completeness is challenging to estimate for masses lower than a few $M_{\odot}$. There is evidence for mass segregation in the cluster (Figer et al. 1999; Stolte et al. 2005), seen as a steepening of the present-day luminosity function toward the cluster center, implying strong spatial selection effects when attempting to constrain the IMF.

Second, the relationship of the PDMF to the IMF is not trivial to evaluate. Stellar evolution must be taken into account when relating the PDMF to the IMF, requiring a prescription for mass loss from high-mass objects (e.g., Espinoza et al. 2009). In addition, mass segregation and tidal stripping are both likely to have been important for the evolution of the Arches. Presentday mass segregation need not be primordial, since the Arches is likely already in a post-collapse phase (e.g., Portegies Zwart et al. 2007; Allison et al. 2009). The Arches cluster sits in a strong tidal field, such that as much as half of its stars may already have been stripped into the field over the $\sim 2.5 \mathrm{Myr}$ of its history (Kim et al. 2000; Portegies Zwart et al. 2002). Mass segregation and tidal stripping together imply that the true IMF of the cluster may differ from the IMF drawn from the subset of stars that have remained within the Arches cluster to the present day. We review the literature mass determinations in Section 5.2.

A kinematic mass measurement provides a direct test of the PDMF of the Arches cluster because its selection effects are somewhat less stringent. Stars below the typical photometric completeness limit of $\sim 1-2 M_{\odot}$ are observable through their contribution to the total cluster mass. Figer et al. (2002) were the first to attempt this, by estimating the radial velocity dispersion of a sample of emission-line stars and assuming that the cluster is spherically symmetric and in virial equilibrium. However, the estimate is complicated by the difficulty of resolving the blended lines, their high width, and intrinsic line profile variation among the sample, so that the resulting mass estimate is strongly dependent on atmosphere models. Mass estimates using the velocity dispersion derived from proper motions are independent of the details of the atmospheres of the tracer stars, and in principle allow for the mass distribution to be derived in a more assumption-free manner (Leonard \& Merritt 1989).

The advent of adaptive optics on large telescopes in the near-infrared has enabled the measurement of precise proper motions of a large number of stars in the GC clusters. In a pioneering proper motion study of the Arches, Stolte et al. (2008) used one epoch each of Very Large Telescope (VLT)/NACO and Keck/Near-Infrared Camera (NIRC2) separated by 4.3 years to measure the motion of the cluster. However, differential distortion between the cameras limited the proper

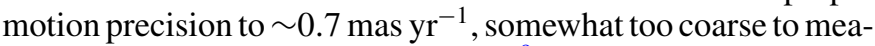
sure the internal velocity dispersion, ${ }^{9}$ for which the expected

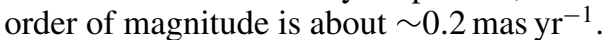

We have observed the central $10^{\prime \prime} \times 10^{\prime \prime}$ of the Arches across five epochs in three years (2006-2009) with a uniform observational setup (PI: Morris). Using NIRC2 on Keck-2, behind the Laser Guide Star Adaptive Optics (LGSAO) facility (Ghez et al. 2005; Wizinowich et al. 2006), these crossinstrument systematics encountered by Stolte et al. (2008) are not present in our observations, and we are able to attain proper motion measurements with error lower than the expected velocity dispersion. We report here on our results, which provide the first kinematic mass estimate of the Arches cluster from proper motions.

This paper is organized as follows. Section 2 describes the observations and positional measurement technique, while Section 3 describes the process of proper motion measurement and error assignment. Section 4 describes the techniques used to fit the cluster membership probabilities and kinematic parameters. Section 5 provides our mass measurement and new bulk motion measurement for the Arches, and briefly discusses the implications.

\section{OBSERVATIONS AND MEASUREMENTS}

Observations of the central $10^{\prime \prime} \times 10^{\prime \prime}$ of the Arches cluster were obtained between 2006 May and 2009 May with the Keck NIRC2 (PI: K. Matthews), behind the LGSAO (van Dam et al. 2006; Wizinowich et al. 2006) system on the W. M. Keck II $10 \mathrm{~m}$ telescope. All observations were obtained with the narrow-field mode of NIRC2 (field of view 10!'2 × 10!'2), which has a pixel scale of $9.952 \pm 0.003$ mas pixel $^{-1}$ (Yelda et al. 2010, hereafter Y10). Observations were taken in the $K^{\prime}$ filter $(\Delta \lambda=0.35 \mu \mathrm{m}$, $\left.\lambda_{0}=2.12 \mu \mathrm{m}\right)$. Five epochs of the central field in $K^{\prime}$ have now been taken (Table 1), the second of which (2006 July 18) was first reported in Stolte et al. (2008). Observations were designed to be as uniform as possible across the epochs, with detector- $Y$ commanded to align with the $\mathrm{S}-\mathrm{N}$ direction at each epoch, with the same pseudorandom dither pattern within a 0 . $7 \times 0.7$ box applied at each epoch (Ghez et al. 2008), and with observations taken at pointings with as uniform a range of zenith angles as practical. Three images were taken at each position within the dither pattern. Figure 1 shows the mean image constructed from the 2009 May data set. This is our best map in terms of both angular resolution (FWHM 51 mas) and

\footnotetext{
9 We use the term "velocity dispersion" to refer to both the dispersion in mas $\mathrm{yr}^{-1}$ and $\mathrm{km} \mathrm{s}^{-1}$ throughout.
} 


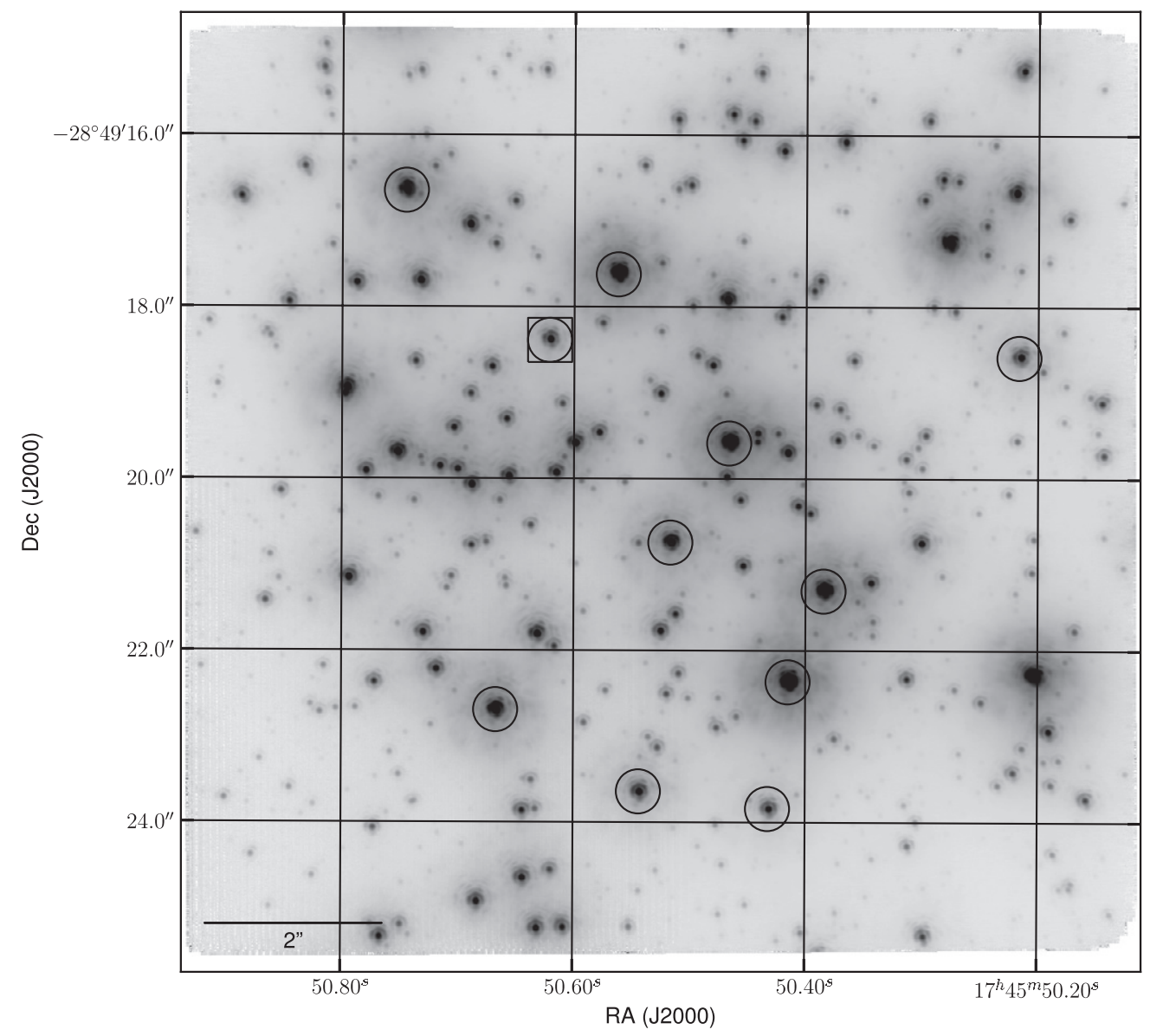

Figure 1. NIRC $2 K^{\prime}$ mosaic of the core field of the Arches in 2009 May. This is our best map in terms of both resolution ( 51 mas) and sensitivity $\left(K_{\text {lim }}^{\prime}=20.59\right.$ mag, Table 1). All stars on which we report in this paper fall within the field of view indicated here. The scale bar is $2^{\prime \prime}$ in length. Stars used as PSF reference stars are indicated by circles. When stellar membership probabilities are reported, positions are reported as offsets from the reference star indicated by the square in this figure.

Table 1

Summary of Observations

\begin{tabular}{lcccccccc}
\hline \hline Epoch & $\begin{array}{c}\left(t_{\text {int }} \times N_{\text {co-add }}\right) \\
(\mathrm{s})\end{array}$ & $N_{\text {images }}$ & $N_{\text {use }}$ & $\begin{array}{c}\text { FWHM } \\
(\mathrm{mas})\end{array}$ & Strehl & $N_{*}$ & $N_{*, \text { uncrowd }}$ & $\begin{array}{c}K_{\text {lim }}^{\prime} \\
(\mathrm{mag})\end{array}$ \\
\hline 2006 May 21 & $3.00 \times 10$ & 15 & 15 & 61.05 & 0.261 & 660 & 649 & 19.43 \\
2006 Jul 18 & $3.00 \times 10$ & 52 & 38 & 56.95 & 0.349 & 657 & 642 & 19.89 \\
2008 May 13 & $3.00 \times 10$ & 146 & 72 & 66.66 & 0.219 & 556 & 536 & 19.74 \\
2008 Jun 1 & $3.00 \times 10$ & 89 & 83 & 54.96 & 0.373 & 845 & 810 & 20.42 \\
2009 May 2 & $2.80 \times 10$ & 119 & 108 & 51.47 & 0.442 & 968 & 917 & 20.59
\end{tabular}

Notes. Reading from left to right, the columns are: epoch of observation, the total integration time for each image, the number of images observed, the number of images used, the median FWHM, the Strehl ratio over the set of accepted images $N_{\text {use }}$, the number of stars measured within the mean image stack in each epoch, the number surviving the cut on proximity to a known neighbor, and finally the magnitude $K_{\text {lim }}^{\prime}$ at which the cumulative distribution function of the observed $K^{\prime}$ magnitudes reaches $90 \%$ of the total number of stars in the sample at each epoch.

sensitivity $\left(K_{\lim }^{\prime}=20.59 \mathrm{mag}\right.$; Table 1$)$. Table 2 shows the point-spread function (PSF) stars used in the analysis.

\section{ANALYSIS}

Our goal is to extract relative proper motions of Arches stars against the field, which is mostly composed of bulge ${ }^{10}$ stars. The analysis proceeds in the following stages. (1) Positions are estimated from a master image at each epoch (Section 3.1). (2) The extracted positions are transformed into a reference

${ }^{10}$ We refer to the bulge/bar system simply as the "bulge" throughout. See Section 5.4 for a brief discussion. frame common to all epochs using likely cluster members, and proper motions extracted from the positional time series in this frame, using statistical uncertainties (Section 3.2). (3) Possible sources of additional proper motion uncertainties are explored (Section 3.3) and, when such additional error sources are identified, motions are re-extracted incorporating the updated errors. Section 3.3 briefly discusses the proper motion precision attained.

\subsection{Production of Star Lists from Each Epoch}

The procedures used by our group to produce lists of stellar positions and fluxes from the image sets at each epoch have been 
Table 2

PSF Stars

\begin{tabular}{lccc}
\hline \hline Row & $\begin{array}{c}K^{\prime} \\
(\mathrm{mag})\end{array}$ & $\begin{array}{l}\Delta x \\
\left({ }^{\prime \prime}\right)\end{array}$ & $\begin{array}{c}\Delta y \\
\left({ }^{\prime \prime}\right)\end{array}$ \\
\hline 1 & 10.24 & 2.736 & -3.943 \\
2 & 10.48 & 2.063 & -1.193 \\
3 & 10.49 & 0.791 & 0.755 \\
4 & 10.66 & 3.150 & -2.899 \\
5 & 11.08 & -0.633 & -4.252 \\
7 & 11.22 & -1.650 & 1.730 \\
8 & 11.25 & 1.385 & -2.334 \\
16 & 12.18 & 1.012 & -5.199 \\
24 & 12.49 & 0 & 0 \\
25 & 12.50 & 5.407 & -0.218 \\
32 & 12.88 & 2.499 & -5.402 \\
\hline
\end{tabular}

Notes. Reading from left to right, the columns are: sequential star number in the master table of membership probabilities, estimated brightness, and finally the position of the star expressed as an offset in arcseconds from the reference star along the (E-W) and (S-N) directions. See Figure 1 for the locations of these stars on the field of view.

fully described elsewhere (Ghez et al. 2008; Lu et al. 2009; Yelda et al. 2010); here we recapitulate briefly the aspects relevant for the present work. Images are calibrated and corrected for differential atmospheric refraction, and corrected for static distortion using the most accurate distortion characterization currently available (Y10). Within an epoch, the corrected images are combined into a mean image using positional shifts only, weighting by the Strehl ratio estimate for each image. Images are combined using the Drizzle algorithm (Fruchter \& Hook 2002), and the mean frame is not supersampled since the pixels already provide 2.5 times Nyquist sampling. This is in contrast to common practice when using Drizzle with Hubble data, which is typically only barely Nyquist sampled. The shifts to use are estimated using cross-correlation of the scene between images. By combining using shifts only, we average through any rapid variations in instantaneous distortion, and average over slow drifts in image orientation, which, based on transformations between common stars across a set of images within the night, appear to be only $\sim 1^{\prime}$ over the course of a night and are therefore negligible. A modified version of the IDL routine Starfinder (Diolaiti et al. 2000) is then used to measure star positions in the mean frame (Y10 and references therein) by simultaneous fitting of the PSF to many stars. In each epoch, 500-900 stars are identified, depending on the performance of the adaptive optics (AO) system and the number of frames collected (Table 1).

Within an epoch, the positional estimate for each star is associated with random measurement uncertainty on the mean of all images within the night that passed quality control (we call this random uncertainty the "centroiding uncertainty;" see Section 3.3.1 for details on its measurement).

\subsection{Transformations to Common Reference Frame and Proper Motion Measurement}

The Arches cluster moves rapidly with respect to the field

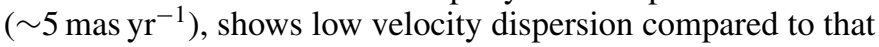
of the field, and accounts for most of the stars in the field of view (Stolte et al. 2008). We therefore measure motions in the reference frame in which the cluster is at rest. ${ }^{11}$ Details of this

\footnotetext{
11 Throughout this discussion the term "frame" refers to reference frames of a
} given epoch or constructed from the positions, not to individual images. process are given in Appendix A; here we outline the important considerations.

First, stars are matched across epochs to produce a master catalog containing all the original position measurements of each star. Each star list is transformed to the frame of a single star list at a chosen epoch $t_{0} .{ }^{12}$ Motions are estimated from straight-line fits to the transformed measurements in $t_{0}$. These measurements are then used to construct a refined cluster reference frame at a chosen time $t_{\text {ref }}$ and the original star lists are then re-mapped onto this refined frame and proper motions re-evaluated in this frame.

The choice of epoch $t_{0}$ is determined by the data quality and by the epochs of observation. Proper motions are determined from straight-line fits to positions in the chosen reference frame; choosing a reference frame near the pivot point of these straightline fits will minimize the error when mapping star lists. Of the three deepest epochs (Table 1), epoch 2008.5 is closest to this pivot point, and is adopted as $t_{0}$. Once a first estimate of proper motions has been produced in this reference frame, the distribution of pivot points of the first pass at straight-line fits is assessed; its mode is $t_{\text {ref }}=2008.0$. The reference frame for motions is then constructed by evaluating the fits to the positional time series from the first pass, at time $t_{\text {ref }}$.

Because the field and cluster populations show significantly different motion, field stars are removed from consideration when evaluating the transformation parameters that map reference frames onto each other. This is achieved by clipping outliers in the vector point diagram; after a few iterations of clipping and re-fitting, the centroid of the cluster population is at zero motion in the vector point diagram.

When mapping star lists between epochs, the transformation parameters are estimated by $\chi^{2}$ minimization using the positional differences in each coordinate separately. The appropriate order of transformation-a second-order polynomial-was determined by evaluating the positional residuals as a function of order (Appendix C). Transformation parameters are given in Table 3.

Measurements are inverse variance weighted using the error estimates in each coordinate for each star. As part of the fitting, three passes of sigma-clipping (with $4 \sigma$ bounds) are used to reduce sensitivity to measurement outliers, mismatches or misidentification of cluster members among the reference stars. This typically removes a few stars from the reference list used for the mapping and can be regarded as a fine-tuning of the reference star list for a given mapping.

As the analysis proceeds, additional estimates of positional error become available to use as weights (see Section 3.3). When mapping star lists onto each other, errors used in the weighting are the positional errors associated with each star list. When mapping star lists onto the reference frame $t_{0}$, these errors are just the centroiding error in each list; when mapping onto $t_{\text {ref }}$ the errors associated with the target frame are the errors in the predicted position in $t_{\text {ref }}$ based on the first pass of motion estimation. When evaluating velocities in a given frame, the positional error and error associated with the mapping into this frame (the alignment error; Section 3.3.2) are added in quadrature. Measured velocities are then used to investigate any additional error not taken into account. Upon discovery of an error source missing from the analysis, the entire analysis is repeated with the missing error term included. Additional 12 In the following discussion, we use " $t_{0}$ " (or " $t_{\mathrm{ref}}$ ") as shorthand for "the
reference frame at time $t_{0}\left(\right.$ or $\left.t_{\mathrm{ref}}\right) . "$ 
Table 3

Transformation Parameters Taking the Star List in Each Epoch into the Reference Frame $t_{\text {ref }}$

\begin{tabular}{|c|c|c|c|c|c|c|}
\hline Parameter & & 2006.38 & 2006.54 & 2008.37 & 2008.50 & 2009.33 \\
\hline$N_{\text {ref }}$ & & 238 & 239 & 235 & 241 & 233 \\
\hline$N_{<4}$ & & 9 & 10 & 21 & 11 & 6 \\
\hline$\Delta$ & $x^{\prime}$ & $-83.94 \pm 6.03 \times 10^{-3}$ & $9.26 \pm 4.01 \times 10^{-3}$ & $4.16 \pm 3.71 \times 10^{-3}$ & $(-4.06 \pm 17.716) \times 10^{-4}$ & $6.53 \pm 3.52 \times 10^{-3}$ \\
\hline (pixel) & $y^{\prime}$ & $-16.88 \pm 4.74 \times 10^{-3}$ & $5.69 \pm 4.39 \times 10^{-3}$ & $3.35 \pm 5.64 \times 10^{-3}$ & $(3.66 \pm 25.421) \times 10^{-4}$ & $3.51 \pm 3.66 \times 10^{-3}$ \\
\hline$x$ & $x^{\prime}$ & $1.00005 \pm 2.11 \times 10^{-5}$ & $0.99994 \pm 9.12 \times 10^{-6}$ & $1.00025 \pm 1.34 \times 10^{-5}$ & $0.99999 \pm 6.18 \times 10^{-6}$ & $1.00002 \pm 8.24 \times 10^{-6}$ \\
\hline () & $y^{\prime}$ & $(6.64 \pm 0.185) \times 10^{-4}$ & $(7.73 \pm 0.079) \times 10^{-4}$ & $(1.33 \pm 1.425) \times 10^{-5}$ & $(2.32 \pm 5.858) \times 10^{-6}$ & $(2.75 \pm 0.090) \times 10^{-4}$ \\
\hline$y$ & $x^{\prime}$ & $(-3.15 \pm 0.177) \times 10^{-4}$ & $(-3.53 \pm 0.089) \times 10^{-4}$ & $(1.23 \pm 0.105) \times 10^{-4}$ & $(-2.40 \pm 4.766) \times 10^{-6}$ & $(-2.54 \pm 0.072) \times 10^{-4}$ \\
\hline () & $y^{\prime}$ & $1.00046 \pm 1.97 \times 10^{-5}$ & $1.00028 \pm 9.62 \times 10^{-6}$ & $1.00014 \pm 1.34 \times 10^{-5}$ & $1.00000 \pm 6.66 \times 10^{-6}$ & $1.00009 \pm 7.99 \times 10^{-6}$ \\
\hline$x^{2}$ & $x^{\prime}$ & $28.90 \pm 5.946$ & $20.61 \pm 3.463$ & $-18.24 \pm 3.989$ & $-0.27 \pm 1.986$ & $4.63 \pm 2.818$ \\
\hline$\left(\times 10^{-8}\right.$ pixel $\left.^{-1}\right)$ & $y^{\prime}$ & $11.78 \pm 4.751$ & $15.66 \pm 3.266$ & $23.10 \pm 4.404$ & $0.48 \pm 1.937$ & $20.65 \pm 2.938$ \\
\hline & $x^{\prime}$ & $1.80 \pm 5.818$ & $20.39 \pm 3.772$ & $58.96 \pm 4.594$ & $-0.28 \pm 2.040$ & $-0.58 \pm 2.973$ \\
\hline$\left(\times 10^{-8}\right.$ pixel $\left.^{-1}\right)$ & $y^{\prime}$ & $-5.05 \pm 6.935$ & $-10.19 \pm 3.985$ & $-28.61 \pm 5.203$ & $-1.52 \pm 2.680$ & $-20.03 \pm 3.335$ \\
\hline & $x^{\prime}$ & $-3.04 \pm 5.917$ & $5.55 \pm 3.048$ & $1.09 \pm 3.540$ & $1.71 \pm 1.686$ & $-0.59 \pm 2.959$ \\
\hline$\left(\times 10^{-8}\right.$ pixel $\left.^{-1}\right)$ & $y^{\prime}$ & $33.22 \pm 6.228$ & $52.07 \pm 3.664$ & $75.11 \pm 5.451$ & $-1.13 \pm 2.488$ & $-0.47 \pm 2.633$ \\
\hline $\begin{array}{l}1.0-M \\
\left(\times 10^{-4}\right) \\
\end{array}$ & & $-2.55 \pm 0.150$ & $-1.14 \pm 0.064$ & $-1.95 \pm 0.095$ & $(1.97 \pm 4.303) \times 10^{-2}$ & $-0.58 \pm 0.062$ \\
\hline $\begin{array}{l}1.0-M_{y} / M_{x} \\
\left(\times 10^{-4}\right)\end{array}$ & & $-4.08 \pm 0.275$ & $-3.34 \pm 0.135$ & $1.10 \pm 0.189$ & $(-5.72 \pm 9.564) \times 10^{-2}$ & $-0.71 \pm 0.104$ \\
\hline $\begin{array}{l}\theta_{\text {rot }} \\
\left({ }^{\prime \prime}\right)\end{array}$ & & $-10.11 \pm 0.250$ & $-11.62 \pm 0.129$ & $1.13 \pm 0.182$ & $(-4.88 \pm 7.925) \times 10^{-2}$ & $-5.46 \pm 0.116$ \\
\hline $\begin{array}{l}\theta_{\text {skew }} \\
\left({ }^{\prime \prime}\right)\end{array}$ & & $7.20 \pm 0.553$ & $8.66 \pm 0.234$ & $2.82 \pm 0.364$ & $-0.002 \pm 0.153$ & $0.43 \pm 0.245$ \\
\hline
\end{tabular}

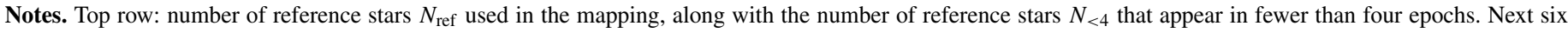

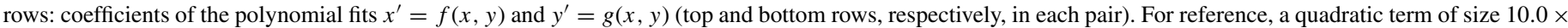

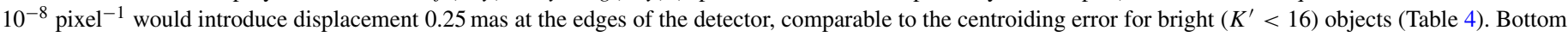

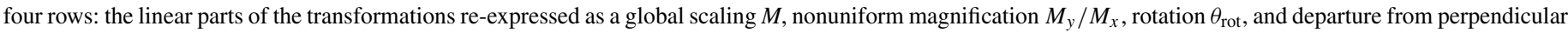
axes $\theta_{\text {skew }}$. (Global shifts $\Delta$ appear in the polynomial fits and are not repeated.)

random error determined from the velocity fits is associated with random variations in position measurement of a given source between epochs, and so is added in quadrature to the centroiding error in the frame mapping and subsequent mapping. The size of this additional error is examined in Section 3.3.3.

\subsection{Positional and Motion Errors}

We now describe the error sources that are included in our analysis in some detail.

\subsubsection{Centroiding Error}

The centroiding uncertainty (random error in position measurement on the mean image from an epoch) is estimated empirically. The stack of images from each epoch is divided into three subsets of equal length, yielding three "submaps"-mean images of each of the three subsets. Images are sorted in decreasing order of Strehl ratio and each submap constructed from every third image surviving quality control in the resulting list. Each submap has therefore been formed from images spanning the same range of observationally relevant conditions (such as Strehl ratio and pointing). Because each submap describes a similar path through auxiliary parameter space both in relation to each other and to the mean image from the night, images are mapped onto the same reference frame using simple translations before averaging into a submap in the same manner as the production of the mean image. Positions are also measured on each of the three submaps in the same way as for the mean image, and these positions then mapped onto the reference frame of the mean image using shifts only. This produces three position measurements from the night, each using one-third of the information from the night and taking the same path through auxiliary parameter space. The rms of stellar position measurements across the three submaps is evaluated for each star to estimate the random error on the mean of one-third of the images within the night. This must be scaled by $1 / \sqrt{3}$ to estimate the centroiding error from the mean image for the star in question. For stars brighter than $K^{\prime}=16$, this centroiding uncertainty is typically 0.1 mas (Table 4 and Figure 2).

\subsubsection{Alignment Error}

Error in predicted positions due to the mapping between frames was estimated through Monte Carlo resampling: sets of half the reference stars were randomly drawn and the framemapping re-fit and re-evaluated for each trial set to produce a trial set of positions as transformed into the target frame. The rms of the differences between these predicted positions and those predicted from the full list is then adopted as the contribution to random positional error due to the fitting process. This error is always included when positional errors after transformation are needed (step 4 and onward in Appendix A). Figure 2 shows the typical magnitude of alignment errors.

\subsubsection{Additional Random Errors}

When velocities were extracted, the distribution of $\chi^{2}$ values from the velocity fits is quite different from that expected if all random errors had correctly been included (Appendix B); clearly additional positional variation is present between epochs 

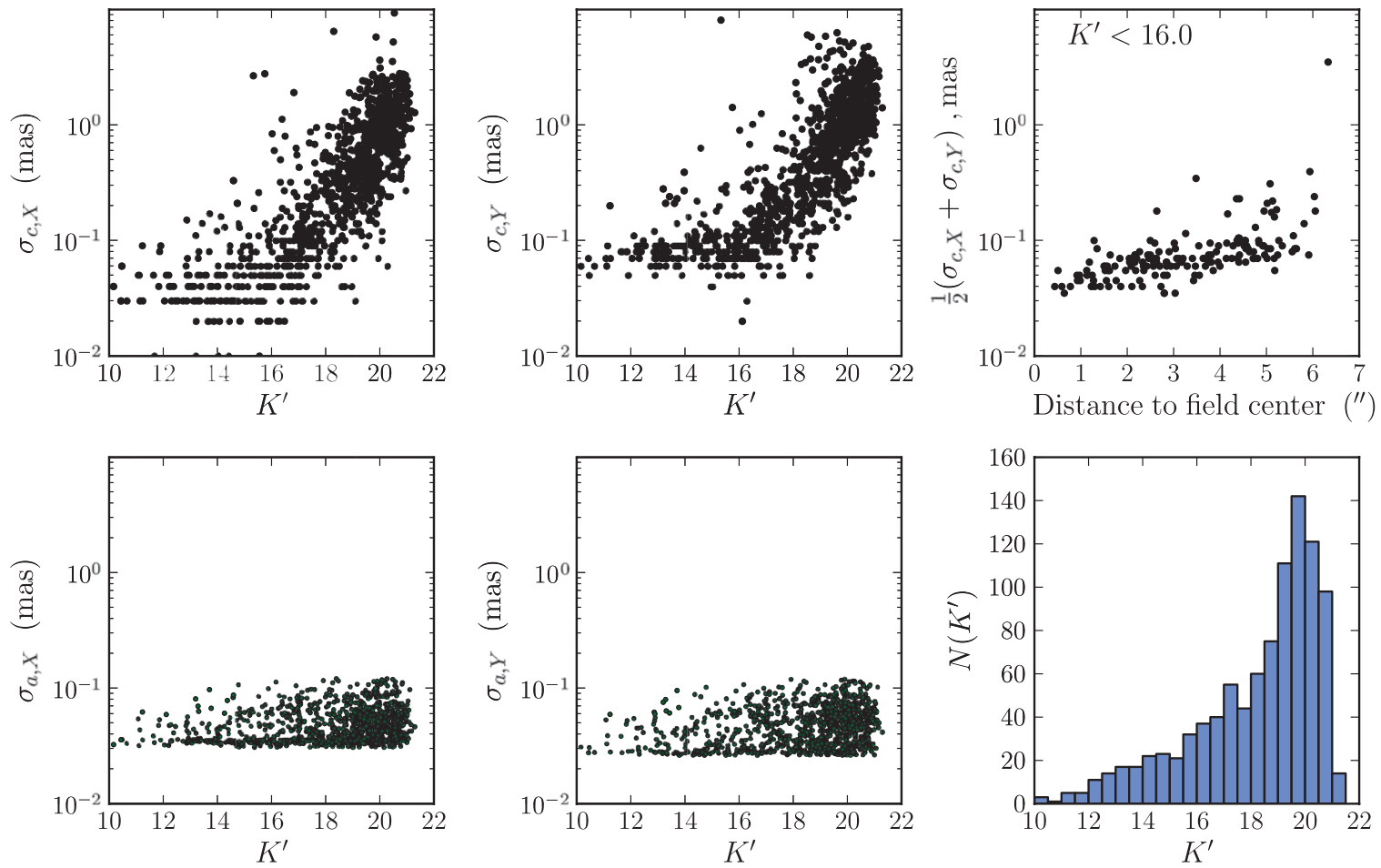

Figure 2. Positional errors as measured for an example epoch (2008.50). Positions are those in the image stack with centroiding errors assessed as the rms of measurements within an epoch (Section 3.3.1). Top row: centroiding errors along detector- $X$ and detector- $Y$ (top left and top middle, respectively), and the average of the two as a function of distance from the field center (top right). Bottom row: alignment errors along $X$ and $Y$ (bottom left and bottom middle, Section 3.3.2). The magnitude histogram is given in the bottom right panel.

(A color version of this figure is available in the online journal.)

Table 4

Astrometric Error Budget

\begin{tabular}{|c|c|c|c|c|c|c|c|c|c|}
\hline \multicolumn{2}{|c|}{$K^{\prime}$} & \multicolumn{5}{|c|}{ Centroiding, Alignment (mas) } & \multirow{2}{*}{$\begin{array}{c}\text { Additive } \\
\text { (mas) }\end{array}$} & \multirow{2}{*}{$\begin{array}{l}\text { Confusion } \\
\text { Bias (mas) }\end{array}$} & \multirow{2}{*}{$\begin{array}{c}\text { Motion } \\
\left(\text { mas yr }^{-1}\right)\end{array}$} \\
\hline & & 2006.39 & 2006.54 & 2008.37 & 2008.50 & 2009.33 & & & \\
\hline \multirow[t]{2}{*}{$10-16$} & $x$ & $0.25,0.09$ & $0.10,0.05$ & $0.25,0.06$ & $0.06,0.04$ & $0.08,0.03$ & $0.16 \pm 0.02$ & \multirow[t]{2}{*}{$0.0-0.03$} & 0.076 \\
\hline & $y$ & $0.23,0.08$ & $0.07,0.05$ & $0.19,0.08$ & $0.11,0.04$ & $0.09,0.05$ & $0.15 \pm 0.02$ & & 0.074 \\
\hline \multirow[t]{2}{*}{$16-18$} & $x$ & $0.41,0.11$ & $0.23,0.05$ & $0.40,0.08$ & $0.14,0.05$ & $0.14,0.04$ & $0.24 \pm 0.02$ & \multirow[t]{2}{*}{$0.03-0.10$} & 0.130 \\
\hline & $y$ & $0.42,0.10$ & $0.20,0.05$ & $0.43,0.09$ & $0.17,0.05$ & $0.18,0.05$ & $0.30 \pm 0.03$ & & 0.153 \\
\hline \multirow[t]{2}{*}{$18-20$} & $x$ & $1.10,0.11$ & $0.92,0.05$ & $1.03,0.08$ & $0.60,0.05$ & $0.59,0.04$ & $0.59 \pm 0.06$ & \multirow[t]{2}{*}{$0.1-1.0$} & 0.378 \\
\hline & $y$ & $1.38,0.10$ & $1.05,0.05$ & $1.35,0.09$ & $0.81,0.05$ & $0.77,0.06$ & $0.71 \pm 0.08$ & & 0.478 \\
\hline
\end{tabular}

Notes. For each magnitude bin, the top (bottom) row gives errors in $X(Y)$. For each star, centroiding, alignment, and additive error describe random variation between epochs. The effect of confusion bias on motions depends on its variation between epochs; random variation is already included in the additive error, while linear trends masquerading as spurious motions are expected to be $\lesssim 10 \%$ of the confusion bias across the epochs for all objects (Section 3.3.3).

that is not accounted for by centroiding and alignment error alone. To properly represent random error along each positional time series, an additional temporally random error $\delta$ (denoted here as "additive error") must be added in quadrature to the random error sources estimated thus far. The size of additive errors $\delta_{x}, \delta_{y}$ required (after accounting for higher-than-linear frame-to-frame transformations; see below) is determined by maximum likelihood, as detailed in Appendix B.1. We find that a flat distribution of additive error with magnitude produces a velocity $\chi^{2}$ distribution significantly more discrepant from statistical expectation than a magnitude-dependent additive error (Appendix B2). We therefore adopt estimates of $\delta_{x}, \delta_{y}$ that vary with magnitude (see Table 4; specifically, for $K^{\prime}<16$ the values are $\delta_{x}, \delta_{y}=0.16,0.15$ mas).

While the balance of important terms varies across different facilities, the major causes of additional error are discussed in some detail by Fritz et al. (2010); we give three example causes here that cannot practically be overcome by experimental design. (1) The AO-corrected PSF core sits on top of a broad halo with radius similar to the seeing radius $(\sim 1 / 20$ th the size of the entire field of view), resulting in significant background spatial structure. Because the spatial scale of this structure is a significant fraction of the field of view, and the field itself is highly crowded, astrometric error due to seeing halo bias is difficult to model with high accuracy. As the seeing halo depends on seeing conditions during the observation, it varies between epochs and therefore manifests itself as an additional time-random error component. ${ }^{13}$ (2) Related to this is source confusion, where the PSF of an object of interest overlaps that

13 This is not the "Halo Noise" of Fritz et al. 2010); they include PSF cores, 
of another object (resolved or unresolved); the magnitude and direction of the bias depends on the relative brightness of the two objects and on the spatial structure of the PSF. Note that this astrometric bias may vary systematically with time (due to time-varying object separation due to object relative motion; e.g., Ghez et al. 2008), or randomly with time due to variation in the PSF structure between epochs (which we assume to be temporally random on timescales of months-years). This confusion error may be spatially correlated if the variation of the PSF between epochs is spatially correlated. (3) The distortion suffered along the path from source to pixels may vary between epochs in a number of ways, producing spatially correlated but temporally random spurious apparent motions between epochs. For example: the mean reference frame within an epoch is constructed from a Strehl-weighted sum of instantaneous images, and therefore depends on the time variation of observing conditions throughout the night, which varies between epochs. Thus, a variation in distortion may be expected between epochs even in the limit of a perfectly stable instrument and telescope. Of the three error sources above, source confusion (source 2) is expected to vary the most strongly with target object magnitude, and thus is a strong candidate for the additive error $\delta_{x}, \delta_{y}$.

\subsection{Resulting Proper Motion Precision}

We have achieved proper motion precision sufficiently high to measure intrinsic dispersion. Positional time series for a selection of objects, along with the motions fitted to the time series of the objects, are provided in Figure 3 . The proper motion precision adopted is shown in Figure 4. Table 4 summarizes the error budget of our astrometric measurements.

\section{RESULTS}

The cluster shares the field of view with a significant field population. To estimate membership probabilities, we fit the kinematic parameters of the cluster and field components simultaneously with the relative contribution each component makes to the population in the image (Section 4.1.1). Armed with membership probabilities, we also estimate the velocity dispersion of the cluster and subsamples within the cluster by direct maximum likelihood fitting of the motions of likely cluster members (Section 4.1.2). Section 4.2 discusses the velocity dispersions resulting from each approach. Since the two approaches produce similar estimates for the dispersion, we reexpress velocities in terms of radial and tangential components in order to calculate the proper motion dispersion profile for use in mass modeling (Section 4.3).

\subsection{Membership Probabilities from Kinematic Fitting}

Table 5 provides a complete catalog of formal membership probabilities for cluster and field for all 432 objects surviving the culls in Appendix A and for which five epochs of position measurement are available. Given best-fit kinematic parameters, the probability that a given star is a member of the $k$ th kinematic component is then the usual

$$
P(k)_{i}=\frac{\phi_{k i}}{\sum_{j}^{K} \phi_{j i}},
$$

where $\phi_{k i}$ gives the likelihood that the $i$ th star belongs to the $k$ th component, and depends on the fit to the kinematic parameters of the field and cluster. We describe the process of obtaining $\phi_{k i}$ below.

\subsubsection{Kinematic Fitting}

Too few field objects are present in our sample to decompose the field population by distance based on our measurements, so we appeal to the literature. The field population is likely dominated by stars in the inner region of the Milky Way bulge and may contain some population from the outer regions of the nuclear stellar disk (hereafter NSD; Launhardt et al. 2002). Both the bulge and NSD should show some degree of central concentration along our line of sight (however, not necessarily centered on the distance of the Arches; see Section 5.3). For the bulge we expect to preferentially sample field stars within a few hundred parsecs of the Arches population itself along the line of sight (e.g., Cabrera-Lavers et al. 2007). The velocity signature of the field component should thus be a sum of differential rotation along the line of sight and intrinsic velocity dispersion, sampled from the bulge and NSD. The contributions of the NSD and Galactic bulge to the field of the Arches may be comparable in size (e.g., Figure 2 of Launhardt et al. 2002), however the uncertainty in the mass model in the inner regions is still rather large. For the purposes of this work, we parameterize the sum of bulge + NSD as a single two-dimensional (2D) Gaussian within the VPD.

Unlike many cluster studies (e.g., Platais et al. 2003), our field component is expected to be significantly asymmetric in the vector point diagram (hereafter VPD), as the bulge velocity dispersion is larger along the Galactic plane than perpendicular to it (Kuijken \& Rich 2002); at 350 pc from the GC, for example, proper motion dispersions are of the order of 5 and 3 mas $\mathrm{yr}^{-1}$ along and perpendicular to the Galactic plane, respectively (Clarkson et al. 2008).

Because the cluster distribution in the VPD is so much tighter than the field distribution, fitting to the binned VPD is not appropriate for this data set as there is no binning factor that allows simultaneous resolution of both cluster and field components. Instead we maximize the likelihood $L$ (data given the component fractions, kinematic parameters, measurement errors) without recourse to binning. The component fraction $\pi_{k}$ describes the proportion of the sample of tracer stars in the image that belong to the $k$ th kinematic component. Since our field fits entirely within the flat core of the surface density radial profile (Espinoza et al. 2009), we assume that $\pi_{k}$ is uniform within our field of view.

We follow standard practice (e.g., Sanders 1971; Jones \& Walker 1988; Kozhurina-Platais et al. 1995) in using a 2D Gaussian profile to model the intrinsic kinematic properties of the cluster in the VPD. The field population is likely to be dominated by bulge objects which occupy a rather narrow distance range compared to the distance to the GC; we therefore parameterize the field component with a 2D Gaussian. Because the convolution of two bivariate Gaussians is another bivariate Gaussian, whose covariance matrix is the sum of those of the two components, this choice of model form allows errors to be included naturally in the analysis. The likelihood of finding a star at a given location in the VPD is thus given by the sum of $K$ Gaussian components:

$$
\begin{aligned}
L\left(\boldsymbol{v}_{i}\right) & =\sum_{k}^{K} \pi_{k} \frac{1}{2 \pi\left|\boldsymbol{\Sigma}_{k i}\right|^{1 / 2}} \exp \left(-\frac{1}{2}\left(\boldsymbol{v}_{i}-\overline{\boldsymbol{\mu}}_{k}\right)^{T} \boldsymbol{\Sigma}_{k i}^{-1}\left(\boldsymbol{v}_{i}-\overline{\boldsymbol{\mu}}_{k}\right)\right) \\
& \equiv \sum_{k}^{K} \phi_{k i},
\end{aligned}
$$



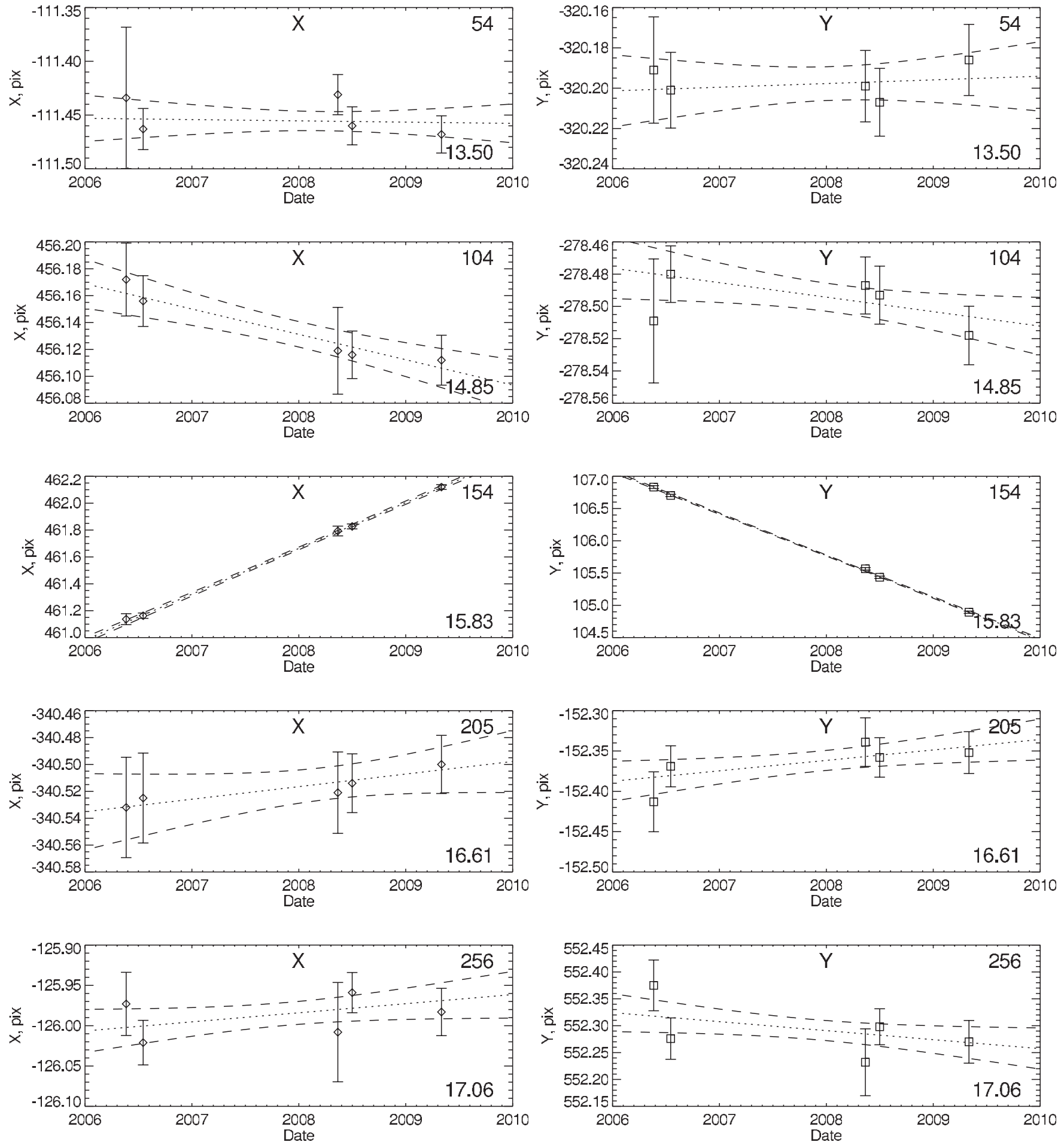

Figure 3. Five example positional time series. Left columns show motion along $X$, right columns along $Y$. Object IDs and $K^{\prime}$ magnitudes are indicated in the right top and bottom corners, respectively. Note that each vertical axis is scaled to accommodate the motion of the star and is in units of pixels in the $t_{\text {ref }}$ reference frame. The best-fit straight line to the motions are indicated in each case, as are $1 \sigma$ positional error curves. Object 154 is likely a field object, as indicated by its large proper motion relative to the reference frame.

where $\phi_{k i}$ describes the likelihood of finding a given star in a given component at its measured location in the VPD. In Equation (2), $\overline{\boldsymbol{\mu}}_{k}$ is the centroid of the $k$ th component in the VPD and $\boldsymbol{\Sigma}_{k i}$ is the covariance of the $k$ th component for the $i$ th star. Because the kinematic model and error model are both Gaussian, the covariances due to error and model combine in the form $\boldsymbol{\Sigma}_{k i}=\mathbf{S}_{i}+\mathbf{Z}_{k}$, where the diagonal positional error matrix $\mathbf{S}_{i}$ has components $\left(\sigma_{v, x}^{2}, \sigma_{v, y}^{2}\right)$ and the covariance matrix of the $k$ th model component is given by $\mathbf{Z}_{k}$.

Once the best-fit $\boldsymbol{\Sigma}_{k i}$ has been estimated from the proper motion data and errors, the intrinsic velocity dispersions $\sigma_{a}, \sigma_{b}$ and the major-axis orientation $\theta$ of each component are found from the eigenvalues and eigenvectors of $\boldsymbol{\Sigma}_{k i}-\mathbf{S}_{i}$.

Best-fit parameters and component fractions are found by maximizing $\sum_{i}^{N} \ln L\left(\boldsymbol{v}_{i}\right)=\sum_{i}^{N} \ln \left(\sum_{k}^{K} \phi_{i k}\right)$ over the sample 

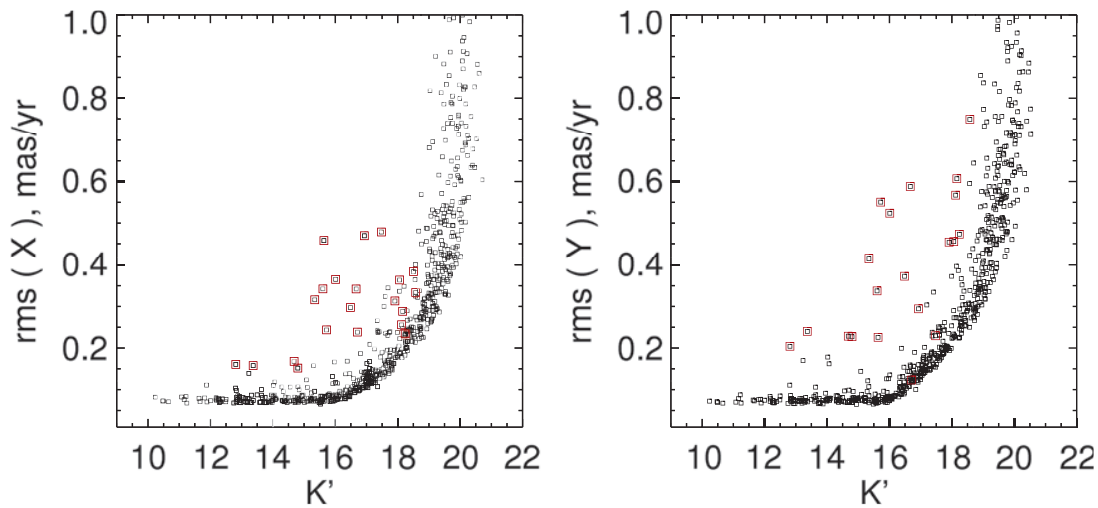

Figure 4. Distribution of adopted proper motion precision (Section 3.3 and Table 4), for all objects with five position measurements. Outliers due to likely mismatches are indicated by squares and were removed from the analysis. An object qualifies as an outlier if the rms in either coordinate falls obviously outside the sequence defined by most of the points.

(A color version of this figure is available in the online journal.)

Table 5

Membership Table for Objects in the Arches Central Field

\begin{tabular}{|c|c|c|c|c|c|c|c|}
\hline Row & $\begin{array}{c}K^{\prime} \\
(\mathrm{mag})\end{array}$ & $\begin{array}{l}\Delta x \\
\left({ }^{\prime \prime}\right)\end{array}$ & $\begin{array}{l}\Delta y \\
\left({ }^{\prime \prime}\right)\end{array}$ & $\begin{array}{c}\mu_{x} \\
\left(\operatorname{mas~yr}^{-1}\right)\end{array}$ & $\begin{array}{c}\mu_{y} \\
\left(\operatorname{mas~yr}^{-1}\right)\end{array}$ & $P$ (Cluster) & $P($ Field $)$ \\
\hline $1^{\mathrm{a}}$ & 10.24 & 2.736 & -3.943 & $0.14 \pm 0.07$ & $0.13 \pm 0.08$ & 0.999 & $8.54 \times 10^{-4}$ \\
\hline $2^{\mathrm{a}}$ & 10.48 & 2.063 & -1.193 & $0.08 \pm 0.07$ & $0.08 \pm 0.07$ & 0.999 & $5.85 \times 10^{-4}$ \\
\hline $3^{\mathrm{a}}$ & 10.49 & 0.791 & 0.755 & $0.15 \pm 0.07$ & $-0.09 \pm 0.07$ & 0.999 & $1.06 \times 10^{-3}$ \\
\hline $4^{\mathrm{a}}$ & 10.66 & 3.150 & -2.899 & $-0.01 \pm 0.07$ & $0.20 \pm 0.07$ & 0.999 & $8.91 \times 10^{-4}$ \\
\hline $5^{\mathrm{a}}$ & 11.08 & -0.633 & -4.252 & $0.03 \pm 0.07$ & $-0.11 \pm 0.08$ & 0.999 & $6.86 \times 10^{-4}$ \\
\hline 6 & 11.16 & 4.603 & 1.092 & $0.00 \pm 0.08$ & $0.07 \pm 0.08$ & 1.000 & $4.88 \times 10^{-4}$ \\
\hline $7^{\mathrm{a}}$ & 11.22 & -1.650 & 1.730 & $0.21 \pm 0.09$ & $0.05 \pm 0.08$ & 0.999 & $1.11 \times 10^{-3}$ \\
\hline $8^{\mathrm{a}}$ & 11.25 & 1.385 & -2.334 & $-0.28 \pm 0.07$ & $0.06 \pm 0.07$ & 0.999 & $1.34 \times 10^{-3}$ \\
\hline 9 & 11.63 & -1.758 & -1.287 & $-0.00 \pm 0.07$ & $0.05 \pm 0.08$ & 1.000 & $4.72 \times 10^{-4}$ \\
\hline 10 & 11.67 & 2.038 & 0.445 & $0.05 \pm 0.07$ & $0.03 \pm 0.07$ & 0.999 & $5.04 \times 10^{-4}$ \\
\hline 11 & 11.81 & -2.337 & -0.540 & $-0.28 \pm 0.08$ & $-0.20 \pm 0.08$ & 0.997 & $2.59 \times 10^{-3}$ \\
\hline 12 & 11.88 & 5.528 & -3.874 & $-0.04 \pm 0.08$ & $0.04 \pm 0.11$ & 1.000 & $4.66 \times 10^{-4}$ \\
\hline 13 & 11.89 & 0.285 & -1.191 & $0.04 \pm 0.07$ & $0.01 \pm 0.07$ & 1.000 & $4.98 \times 10^{-4}$ \\
\hline 14 & 12.00 & -0.158 & -3.382 & $-0.06 \pm 0.07$ & $-0.08 \pm 0.07$ & 0.999 & $5.54 \times 10^{-4}$ \\
\hline 15 & 12.18 & 5.362 & 1.667 & $-0.02 \pm 0.08$ & $0.09 \pm 0.08$ & 0.999 & $5.02 \times 10^{-4}$ \\
\hline $16^{\mathrm{a}}$ & 12.18 & 1.012 & -5.199 & $0.02 \pm 0.07$ & $-0.13 \pm 0.08$ & 0.999 & $7.41 \times 10^{-4}$ \\
\hline 17 & 12.19 & -1.490 & 0.681 & $0.06 \pm 0.08$ & $-0.07 \pm 0.07$ & 0.999 & $6.26 \times 10^{-4}$ \\
\hline
\end{tabular}

Notes. Reading left to right, the columns are: sequential star number, estimated brightness, offset from reference star (E-W and S-N), proper motion and error, and the formal probability that the object is associated with the cluster and field, respectively.

${ }^{a}$ PSF star.

(This table is available in its entirety in a machine-readable form in the online journal. A portion is shown here for guidance regarding its form and content.)

of tracer stars of interest, under the constraint $\sum_{k}^{K} \pi_{k}=1$. The maximum likelihood $\pi_{k}$ and the kinematic parameters are evaluated sequentially and iteratively until convergence. If the errors are constant over the sample of interest (so $\boldsymbol{\Sigma}_{k i} \approx \mathbf{Z}_{k}+\overline{\mathbf{S}}$ ), then each update step requires the evaluation of analytic expressions for the maximum likelihood estimate of the updated $\pi_{k}^{\prime}$ given the current estimate of the parameters (and vice versa). This is the well-known expectation maximization (hereafter EM) algorithm. This technique is well established outside astronomy (Chapter 9 of Bishop 2006 provides a thorough explanation, and the method has appeared in the most recent edition of Press et al. 2002) and is becoming more commonly employed to mixture problems in astronomy in which binning is undesirable and/or a low number of reference objects is available (Bovy et al. 2011).

Strongly varying error on a star-by-star basis is a significant complication, as the parameter-update step no longer has an analytic form, and instead must be solved numerically. For the present investigation, we use a single cluster component and choose instead a variant of the technique of KozhurinaPlatais et al. (1995 and references therein) in which membership probabilities for each star are estimated using kinematic parameters fitted only from stars with roughly similar error (so $\boldsymbol{\Sigma}_{k i} \approx \mathbf{Z}_{k}+\overline{\mathbf{S}}=\boldsymbol{\Sigma}_{k}$ for the subsample). The sample is broken into overlapping bins two magnitudes wide (so $K^{\prime}=14.0-16.0$, $15.0-17.0,16.0-18.0$, and 17.0-19.0) and the best-fit parameters determined for each magnitude strip separately (Table 6 and Figure 5). Investigation of remaining magnitude-dependent bias can be found in Appendix D. We find that parameters do not become strongly affected by bias until stars as faint as $K^{\prime}=18$ are considered.

Between $15 \leqslant K^{\prime}<18$, every star is at most half a magnitude from the center of one of the magnitude strips, and it is the parameters corresponding to this nearest magnitude strip that are used to evaluate Equation (1) for these stars. Objects at $K^{\prime}<15.0$ use the kinematic parameters evaluated for 
Table 6

Fitted Kinematic Parameters of Cluster and Field

\begin{tabular}{|c|c|c|c|c|c|}
\hline$K^{\prime}$ & $14.0-16.0$ & $15.0-17.0$ & $16.0-18.0$ & $17.0-19.0$ & $18.0-20.0$ \\
\hline$N$ & 75 & 105 & 135 & 165 & 135 \\
\hline$\pi_{\mathrm{cl}}$ & $0.80 \pm 0.036$ & $0.72 \pm 0.035$ & $0.72 \pm 0.030$ & $0.59 \pm 0.029$ & $0.52 \pm 0.032$ \\
\hline $\begin{array}{l}\Delta \mu \\
\left(\operatorname{mas~yr}^{-1}\right)\end{array}$ & $4.28 \pm 0.526$ & $4.54 \pm 0.430$ & $5.15 \pm 0.356$ & $3.68 \pm 0.293$ & $3.51 \pm 0.320$ \\
\hline $\begin{array}{l}\phi_{f} \\
\left({ }^{o}\right) \\
\end{array}$ & $30.9 \pm 4.8$ & $30.5 \pm 3.9$ & $37.1 \pm 3.1$ & $36.6 \pm 2.5$ & $32.5 \pm 2.7$ \\
\hline $\begin{array}{l}\sigma_{a, f} \\
\left(\text { mas yr }^{-1}\right) \\
\end{array}$ & $2.21 \pm 0.338$ & $2.56 \pm 0.280$ & $2.87 \pm 0.240$ & $2.89 \pm 0.198$ & $2.80 \pm 0.216$ \\
\hline $\begin{array}{l}\sigma_{b, f} \\
\left(\operatorname{mas~}^{-1} r^{-1}\right)\end{array}$ & $1.50 \pm 0.231$ & $1.64 \pm 0.193$ & $1.85 \pm 0.159$ & $1.81 \pm 0.130$ & $1.64 \pm 0.137$ \\
\hline $\begin{array}{l}\sigma_{a, \mathrm{cl}} \\
\left(\mathrm{mas} \mathrm{yr}^{-1}\right)\end{array}$ & $0.15 \pm 0.013$ & $0.17 \pm 0.012$ & $0.16 \pm 0.014$ & $0.24 \pm 0.022$ & $0.45 \pm 0.034$ \\
\hline $\begin{array}{l}\sigma_{b, \mathrm{cl}} \\
\left(\mathrm{mas} \mathrm{yr}^{-1}\right)\end{array}$ & $0.12 \pm 0.010$ & $0.16 \pm 0.010$ & $0.16 \pm 0.012$ & $0.16 \pm 0.019$ & $0.17 \pm 0.029$ \\
\hline $\begin{array}{l}\theta_{f} \\
\left(^{o}\right) \\
\end{array}$ & $33.9 \pm 17.3$ & $27.8 \pm 14.2$ & $35.0 \pm 11.8$ & $28.5 \pm 8.8$ & $26.7 \pm 10.3$ \\
\hline $\begin{array}{l}\theta_{\mathrm{cl}} \\
\left({ }^{o}\right) \\
\end{array}$ & $70.2 \pm 21.7$ & $78.6 \pm 30.3$ & $67.5 \pm 48.3$ & $117.1 \pm 63.5$ & $114.0 \pm 66.4$ \\
\hline$\underline{\sigma_{b, \mathrm{cl}} / \sigma_{a, \mathrm{cl}}}$ & $0.83 \pm 0.086$ & $0.91 \pm 0.078$ & $0.96 \pm 0.069$ & $0.69 \pm 0.075$ & $0.37 \pm 0.085$ \\
\hline$\sigma_{b, f} / \sigma_{a, f}$ & $0.68 \pm 0.130$ & $0.64 \pm 0.113$ & $0.65 \pm 0.099$ & $0.63 \pm 0.083$ & $0.58 \pm 0.090$ \\
\hline
\end{tabular}

Notes. For each magnitude range, rows give the cluster fraction, the separation between cluster and field centers in the vector point diagram (VPD), the orientation of the separation vector from the cluster center to the field center in the VPD, the semimajor and minor axes of the field component, the semimajor and minor axes of the cluster component, the orientation of the semimajor axis of the field component, the orientation of the semimajor axis of the cluster component, and finally the axis ratio (minor/major) of the cluster and field components. Errors are estimated from Monte Carlo simulations: populations in the VPD are simulated under the intrinsic kinematic parameters estimated from observation, perturbed by the measured errors for stars in each magnitude range, and re-fitted. Orientations are position angles reported in degrees east of north.

$K^{\prime}<16.0$ (see also Section 4.1.2), while objects at $K^{\prime}>18.0$ use the kinematic parameters estimated from $17.0 \leqslant K^{\prime}<19.0$.

Errors on the parameters thus fit are estimated by Monte Carlo bootstrap analysis. The observed VPD is resampled by random drawing of points from the full sample with replacement. The full kinematic fitting process is re-applied to each trial and the distribution of recovered values parameterized with its standard deviation about the mean value using the full data set.

\subsubsection{Direct Calculation of the Velocity Dispersion}

The process given in Section 4.1.1 fails when the sample size is small (i.e., $\lesssim 70$ stars), or contains a negligible field component (as occurs for $K \lesssim 14.5$ ). Our end goal is to compute the dispersion profile as a function of distance from the cluster center for mass modeling, which may entail few stars per annulus. We therefore compute the velocity dispersion from proper motions using a direct maximum likelihood approach. Likely cluster objects are isolated using the membership probabilities estimated from the fits of the previous section. Along each direction, we maximize

$L(\bar{v}, \sigma)=\prod_{i}^{N}\left(2 \pi\left(\sigma^{2}+e_{i}^{2}\right)\right)^{-1 / 2} \exp \left\{-\left(v_{i}-\bar{v}\right)^{2} / 2\left(\sigma^{2}+e_{i}^{2}\right)\right\}$

for $\bar{v}, \sigma$ iteratively, where $\sigma$ is the intrinsic velocity dispersion and $e_{i}$ is the measurement error. For each $\sigma, \bar{v}$ is obtained by weighted averaging while for each $\bar{v}, \sigma$ is found numerically by bisection. Errors are estimated by Monte Carlo bootstrap analysis; members of the subsample are drawn randomly with replacement and the calculation repeated for a large number of trials. The standard deviation of the fitted parameters across the set of trials is then adopted as the fitting error from this process. Table 7 shows the dispersions and their errors estimated by this procedure.

\subsection{Cluster Velocity Dispersion}

The kinematic fitting (Section 4.1.1) and direct (Section 4.1.2) approaches produce complementary estimates for the intrinsic velocity dispersion of the Arches cluster. Both have been corrected for measurement error using the procedures described above. Tables 6 and 7 show the kinematic parameters fit to cluster and field, and the velocity dispersion estimate for the cluster, respectively. Figure 6 shows the velocity dispersion graphically and its comparison to the proper motion error.

Both estimates yield statistically significant measurements of the cluster velocity dispersion. The mean cluster velocity dispersion and its error is estimated by inverse varianceweighted average of the determinations from non-overlapping magnitude bins, for $K^{\prime}<18.0$ (to avoid strong error and mass segregation effects). For the dispersions estimated from the kinematic fitting (Section 4.1.1 and Table 6), magnitude bins $\left(14.0 \leqslant K^{\prime}<16.0\right)$ and $\left(16.0 \leqslant K^{\prime}<18.0\right)$ are used. We do not use the magnitude bin $K^{\prime}>18.0$ because this bin appears to suffer misclassification bias under the fitting technique used; see Appendix D. 
Table 7

Arches Velocity Dispersion in Each Coordinate

\begin{tabular}{lrcccc}
\hline \hline$K^{\prime}$ & $N$ & $\begin{array}{c}\sigma_{x} \\
\left(\mathrm{mas} \mathrm{yr}^{-1}\right)\end{array}$ & $\begin{array}{c}\sigma_{y} \\
\left(\mathrm{mas} \mathrm{yr}^{-1}\right)\end{array}$ & $\begin{array}{c}\sigma_{x} \\
\left(\mathrm{~km} \mathrm{~s}^{-1}\right)\end{array}$ & $\begin{array}{c}\sigma_{y} \\
\left(\mathrm{~km} \mathrm{~s}^{-1}\right)\end{array}$ \\
\hline $10.0-14.0$ & 67 & $0.130 \pm 0.017$ & $0.123 \pm 0.016$ & $4.912 \pm 0.639$ & $4.680 \pm 0.593$ \\
$14.0-16.0$ & 72 & $0.161 \pm 0.019$ & $0.129 \pm 0.016$ & $6.088 \pm 0.739$ & $4.878 \pm 0.606$ \\
$16.0-18.0$ & 107 & $0.177 \pm 0.027$ & $0.180 \pm 0.030$ & $6.721 \pm 1.034$ & $6.839 \pm 1.142$ \\
$18.0-20.0$ & 97 & $0.224 \pm 0.039$ & $0.148 \pm 0.046$ & $8.508 \pm 1.498$ & $5.629 \pm 1.753$ \\
\hline
\end{tabular}

Notes. Reading from left to right, the columns are: magnitude range of interest, number of cluster stars in this magnitude

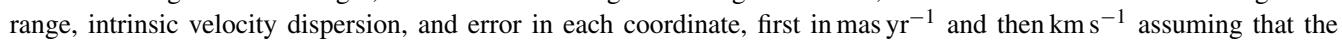
Arches is at $8.4 \mathrm{kpc}$.

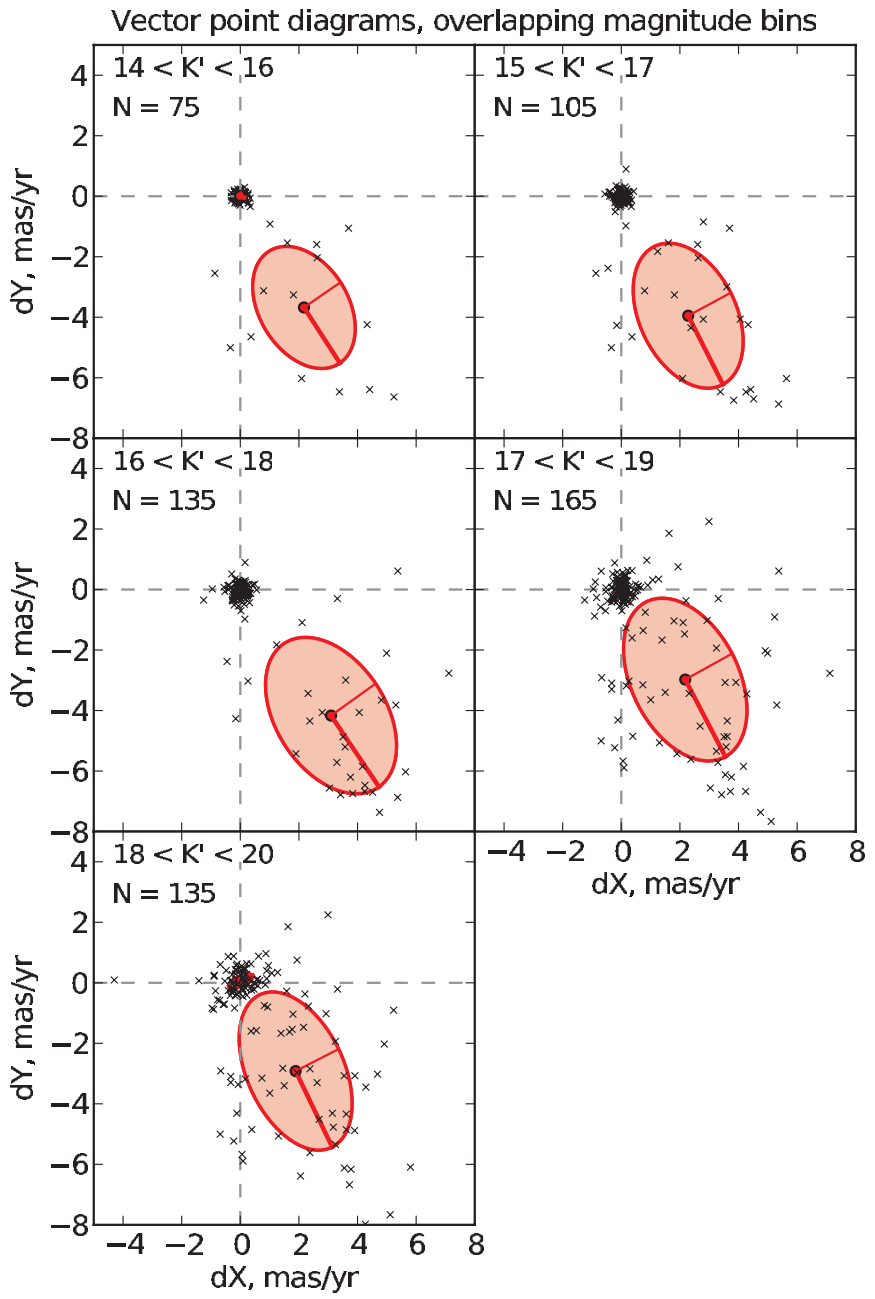

Figure 5. Vector point diagrams for the overlapping magnitude ranges of Section 4.1.1 and Table 6. Shaded ellipses give the $1 \sigma$ contours for the 2D Gaussian components fit to the field and cluster components. Within each ellipse, the lines indicate the length and direction of the semimajor (thick red line) and semiminor (thin red line) axes.

(A color version of this figure is available in the online journal.)

This yields mean velocity dispersions along major and minor axes respectively, ${ }^{14}$ of $\left(\overline{\sigma_{a}}, \overline{\sigma_{b}}\right)=(0.154 \pm$ $0.01,0.136 \pm 0.008) \mathrm{mas} \mathrm{yr}^{-1}$. Scaling to the distance of the GC $(8.4 \pm 0.4 \mathrm{kpc}$; Ghez et al. 2008) this yields measured velocity dispersion $(5.8 \pm 0.48,5.2 \pm 0.40) \mathrm{km} \mathrm{s}^{-1}$.

For the dispersions estimated directly from likely cluster members in each direction separately (Section 4.1.2 and

${ }^{14}$ Not Galactic longitude and latitude.
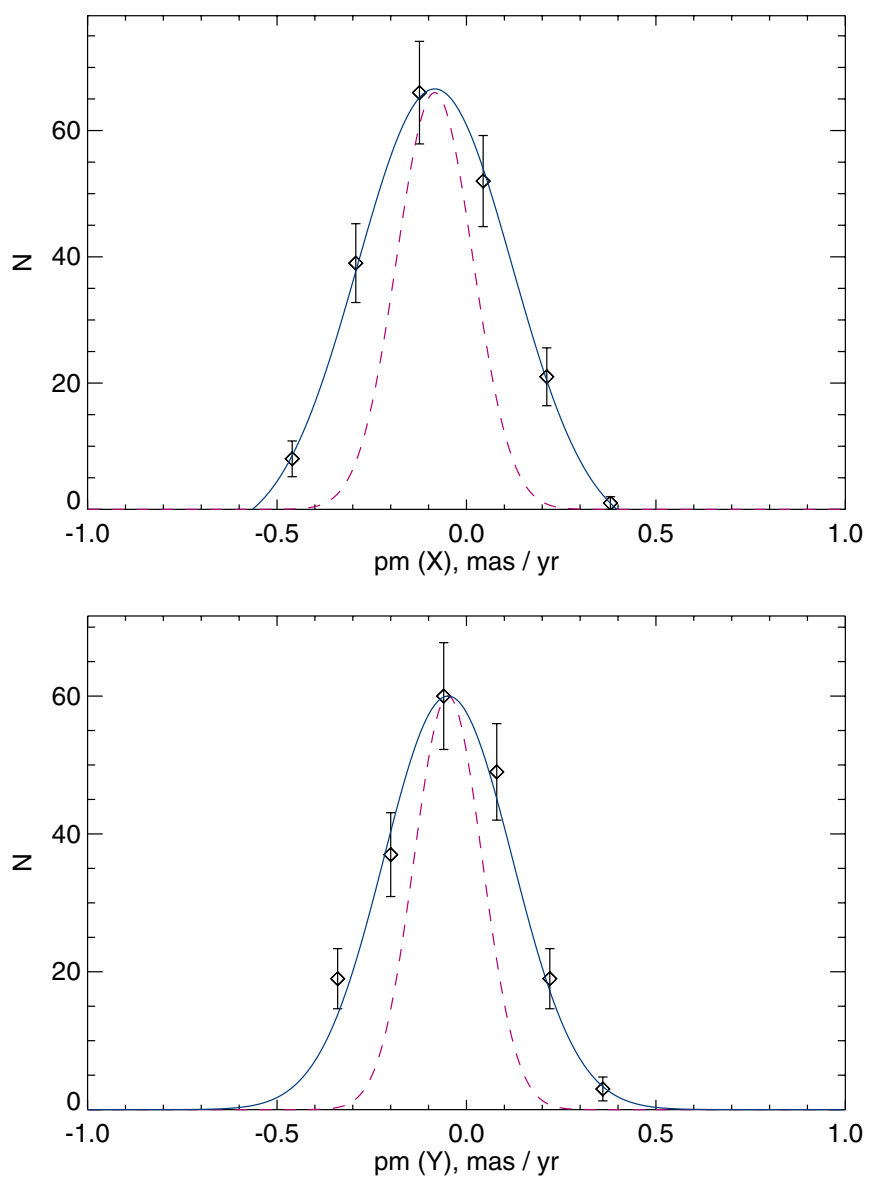

Figure 6. Observed velocity dispersion in each coordinate for stars with $14.0 \leqslant K^{\prime}<17.0$ compared to a Gaussian of width equal to the mean measurement error over this range (Section 3.3 and Table 4). Panels correspond to detector- $X$ (top) and detector- $Y$ (bottom). This figure was constructed after removing likely field objects (Section 4.1).

(A color version of this figure is available in the online journal.)

Table 7), the mean velocity dispersions in the three nonoverlapping bins brighter than $K^{\prime}=18.0$ are $\left(\overline{\sigma_{x}}, \overline{\sigma_{y}}\right)=$ $(0.149 \pm 0.011,0.124 \pm 0.016) \mathrm{mas}^{-1}$, which translates to velocity dispersion $(5.7 \pm 0.51,4.68 \pm 0.65) \mathrm{km} \mathrm{s}^{-1}$ along detector $X Y$ coordinates.

Included in Table 6 is the position angle (east of north in the VPD) of the major axis of the cluster component (denoted $\theta_{\mathrm{cl}}$ ). Comparing independent magnitude bins, we see that the orientation of the cluster major axis changes from $70.2 \pm 21.7$ to $67.5 \pm 48.3$ to $114.0 \pm 66.4$ as fainter magnitude bins are considered. Such an extreme variation indicates the apparent variation in the cluster major-axis orientation is likely a sampling 

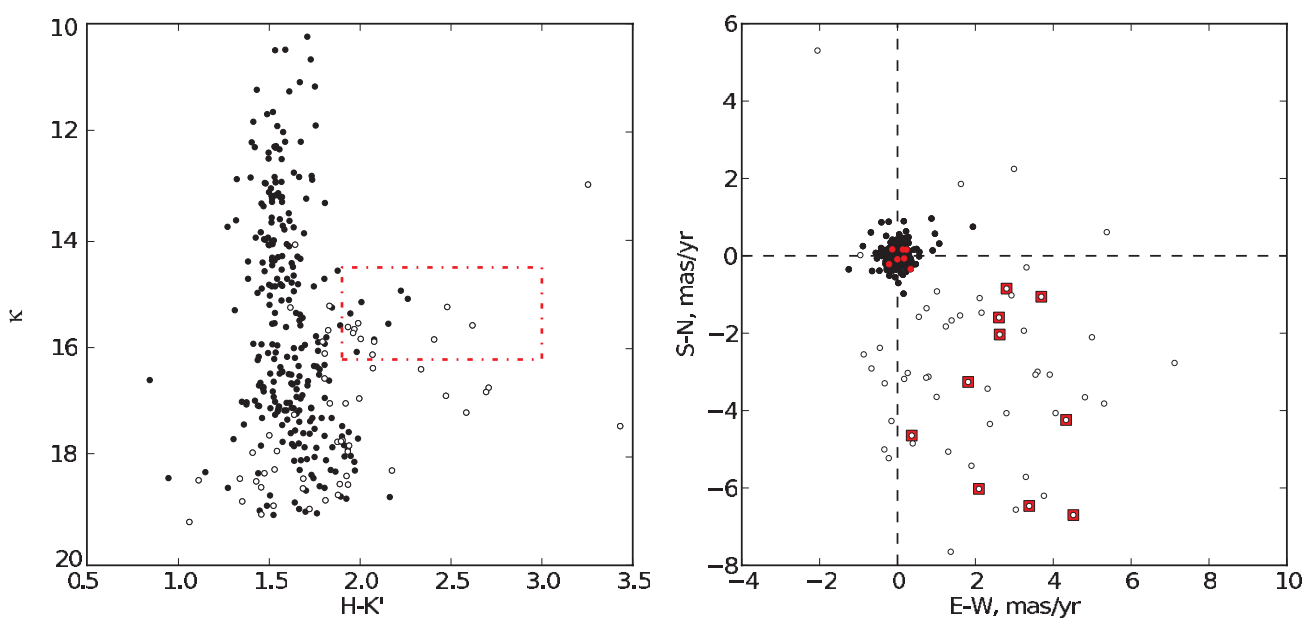

Figure 7. Color-magnitude diagram (CMD) and vector point diagram (VPD) for all objects with proper motion error $<0.5$ mas $^{-1} \mathrm{y}^{-1}$ and five epochs of measurement. The CMD presented here was constructed by matching $K^{\prime}$ measurements to photometry taken in the $H$ band in 2006 May with Keck-2/NIRC2-LGS (McCrady et al. 2011), which limits the depth in the CMD. Objects with $P_{\text {cluster }}>0.995$ are shown in black; all other objects denoted with open circles. Red objects in the VPD correspond to the stars within the red dot-dashed box in the CMD, and represent well-measured objects with a possible $H-K^{\prime}$ excess. Of these objects, those with $P_{\text {cluster }}>0.995$ are shown with a red circle; their field counterparts are shown with red squares. See Section 5.5 and Stolte et al. (2010) for more information on these objects.

(A color version of this figure is available in the online journal.)

artifact, and does not reflect underlying variation. The detector $X$ - and $Y$-directions therefore sample a symmetric distribution in two directions that are arbitrary with respect to the cluster velocity distribution. We can therefore compare the two measures by averaging the major and minor axes from kinematic fitting to make a direction-invariant measure from kinematic fitting, and average the directly calculated dispersions along the two detector directions to form a second direction-invariant dispersion measure. This yields mean velocity dispersions $5.4 \pm 0.3 \mathrm{~km} \mathrm{~s}^{-1}$ and $5.5 \pm 0.4 \mathrm{~km} \mathrm{~s}^{-1}$, respectively, for dispersions estimated from kinematic fitting $\left(14.0 \leqslant K^{\prime}<\right.$ 18.0) and those estimated directly from cluster members $\left(10.0 \leqslant K^{\prime}<18.0\right)$.

\subsection{Velocities for Mass Estimates}

Sections 4.1.1 and 4.1.2 establish that mixture modeling (used to establish membership probabilities) and direct calculation from cluster members produce the same estimate of the dispersion. To be compatible with mass estimates based on kinematic modeling, proper motions expressed in components aligned with the detector axes are converted into proper motion components along the radial vector away from the cluster center, and along the vector tangential to it. Specifically, the velocities and their errors used for mass estimates, $v_{R}, v_{T}, \delta_{R}, \delta_{T}$, are computed from the following relations:

$$
\begin{aligned}
\theta & \equiv \arctan \frac{Y_{*}-Y_{0}}{X_{*}-X_{0}} \\
v_{R} & =v_{X} \cos \theta+v_{Y} \sin \theta \\
v_{T} & =-v_{X} \sin \theta+v_{Y} \cos \theta \\
\delta_{R}^{2} & =\delta_{X}^{2} \sin ^{2} \theta+\delta_{Y}^{2} \cos ^{2} \theta \\
\delta_{T}^{2} & =\delta_{X}^{2} \cos ^{2} \theta+\delta_{Y}^{2} \sin ^{2} \theta,
\end{aligned}
$$

where $v_{X}, v_{Y}, \delta_{X}, \delta_{Y}$ are the velocities and their errors along detector- $X$ and detector- $Y$ that were estimated from proper motions. The positions $X_{*}, Y_{*}$ denote the position of the star on the detector, and $X_{0}, Y_{0}$ denote the location of the cluster center on the detector. The sample is then broken into concentric annuli,

\begin{tabular}{|c|c|c|c|}
\hline \multicolumn{2}{|c|}{ Offset from Star 24} & \multicolumn{2}{|c|}{ Offset from Field Center } \\
\hline$\Delta_{\mathrm{E}-\mathrm{W}}$ & $\Delta_{\mathrm{S}-\mathrm{N}}$ & $\Delta_{\mathrm{E}-\mathrm{W}}$ & $\Delta_{\mathrm{S}-\mathrm{N}}$ \\
\hline$+1^{\prime \prime} .5$ & $-1{ }^{\prime \prime} .5$ & $+0 ! 23$ & +0.22 \\
\hline$+1^{\prime \prime} .5$ & -2 .'5 & $+0 ! 23$ & -0.79 \\
\hline+2.5 & -2.5 & $+1^{\prime \prime} .23$ & -0.79 \\
\hline$-0,5$ & $-3^{\prime \prime} .5$ & $-1^{\prime \prime} .77$ & $-1^{\prime \prime} .79$ \\
\hline
\end{tabular}

Table 8

Location of the Field Centers Chosen to Evaluate the Radial and Transverse Components of the Velocity Dispersion from Proper Motions (Section 4.3; See Also Section 5.1)

Notes. The left column gives positions as offsets (E-W) and (S-N) from the reference star marked in Figure 1 (see also Tables 2 and 5). The right column gives offsets from the center of the field of view.

and the velocity dispersion and its error within each annulus computed by the method of Section 4.1.2. To select a sample of cluster member stars, formal membership probabilities $P_{\text {cluster }}>$ 0.995 were used (see Figure 7 for an illustration of the VPD and color-magnitude diagram (CMD) using this membership probability as a cutoff).

The cluster center itself is not apparent as a peak in individual motions or surface density within the restricted field of view of our central-field observations (compare with Espinoza et al. 2009; Anderson \& van der Marel 2010), although the dynamical center of the cluster probably is within the rough center of our field of view. Four randomly chosen locations for the cluster center $X_{0}, Y_{0}$ are chosen (Table 8), all within 2".6 from the center of the field. Mass limits reported in Section 5.1 are taken from the ensemble range over all four choices of cluster center; in practice, the range of derived masses is not strongly dependent on the location of the cluster center.

\section{DISCUSSION}

A key goal of this work is to compare mass limits set by proper motion dispersion measurements to literature mass estimates. The set of literature mass estimates is quite heterogeneous, so some discussion of notation is in order before proceeding. 
We use $\Gamma_{0}$ to represent the IMF slope where quoted in the cited report, and $\Gamma$ to represent the PDMF; both exponents refer to the form $d N / d(\log M) \propto M^{-\Gamma}$. Simulations (e.g., Kim et al. 2000; Kim \& Morris 2003) suggest that the Arches may have lost about half its mass since formation due to dynamical effects; we therefore distinguish between present-day mass $M_{\mathrm{cl}}$ and initial mass $M_{0}$ in the following discussion. We use radius $r$ to refer to a distance from the cluster center in three dimensions and $R$ to refer to distance from the cluster center as projected onto the sky. The term "half-mass radius" refers to the radius within which half the cluster mass is found, but the precise meaning of this term often depends on the application. In our notation, $r_{\mathrm{hm}}$ refers to the radius of a sphere within which half the cluster mass is contained (the sense often used by theorists; Portegies Zwart et al. 2010) and $R_{\mathrm{hm}}$ is the radius of a cylinder oriented along the line of sight, within which half the cluster mass (usually the mass of the directly observed tracer stars) is observed. This latter quantity is the half-mass radius most commonly seen in observational estimates. ${ }^{15}$ Where a total cluster mass is reported, we denote it as $M_{\mathrm{cl}}$ or $M_{0}$; where mass within a given projected radius is reported as $M(R<0.4 \mathrm{pc})$ so that the outer radius limit is clear. Throughout this report the term "projected mass" refers to the mass within a cylinder of radius $R$ on the sky whose long axis is aligned along the line of sight.

\subsection{Velocity Dispersion and Mass}

The only previous use of stellar motions to estimate the mass of the Arches of which we are aware is that of Figer et al. (2002). Radial velocities of eight emission-line stars within $R=0.23$ pc of the cluster center were used to place an upper limit on the one-dimensional velocity dispersion. Their $22 \mathrm{~km} \mathrm{~s}^{-1}$ was converted to an upper limit on the enclosed mass using the virial theorem; a spherically symmetric, gravitationally bound cluster with this velocity dispersion would have enclosed mass $M_{\mathrm{cl}}(r<0.23 \mathrm{pc})=7 \times 10^{4} M_{\odot}$. For such massive stars with strong stellar winds, interpretation of the line profile shape to estimate systematic velocities is strongly dependent on model atmospheres for massive stars (Figer et al. 2002).

A mass estimate based on proper motions is independent of the details of the line profile of the young, massive stars to which we are sensitive. We choose not to employ the momentbased kinematic mass estimator of Leonard \& Merritt (1989, hereafter LM89), since its power to estimate the total mass given the projected-radial coverage is strongly sensitive to the outer radius limit (e.g., Figure 11 of Schödel et al. 2009).

The method used is as follows: We start with a model for the mass density profile $\rho(r)$ of the cluster, whose parameters are varied to evaluate the comparison to our proper motion-based dispersion estimates. Several model choices are possible. We choose to use the King (1962) prescription to describe the radial density profile $\rho(r)$ of the cluster. Our choice is motivated by three observations from the literature. First, the Arches cluster is likely already in a post-collapse phase; simulations suggest that for the Arches cluster, core collapse takes place only about $0.6 \mathrm{Myr}$ after formation (Kim et al. 1999). This collapse erases the signature of substructure in the parent cloud and leads to cluster relaxation on a shorter timescale than the initial halfmass relaxation time. Second, the resulting cluster undergoes dynamical mass segregation on a timescale of 1-2 Myr, much shorter than suggested by the current crossing time of most stars

\footnotetext{
15 For example, our $R_{\mathrm{hm}}$ is the same as the quantity $r_{\mathrm{hm}}$ found in Figer et al. (1999) and Stolte et al. (2002).
}

in the cluster (Allison et al. 2009). The cluster is thus much older dynamically than its current crossing time would suggest (Bastian et al. 2008; Allison et al. 2009). This suggests that a model assuming a relaxed cluster should be a reasonable first approximation to the Arches, even given its young age. Third, the observed surface density profile $\Sigma(R)$ (units stars $\mathrm{pc}^{-2}$ ) of massive stars $\left(M>10 M_{\odot}\right)$ is indeed reasonably well fit by a King (1962) profile (Espinoza et al. 2009).

To use the King (1962) model to predict observed velocity dispersions, we make four further assumptions; we anticipate that the next step in the analysis will be to move straight to full numerical modeling of the cluster, but that is beyond the scope of this paper. We assume that the cluster is (1) not rotating strongly, (2) can be characterized as being in equilibrium, (3) has spherical symmetry, and (4) shows isotropic motion. Assumption (2) allows us to use the Jeans equation to predict the velocity dispersion profile $\sigma^{2}(r)$ corresponding to each model parameter set. This profile is then projected onto the sky to predict the observed velocity dispersion components parallel and perpendicular to the radial direction away from the cluster center. This prediction is compared to observations (Section 4.3), and a figure of merit $\left(\chi^{2}\right)$ evaluated for each set of parameters. The set of $\chi^{2}$ values mapped out in this way is used to derive confidence limits for the model parameters. Finally, confidence limits on the model parameters are converted into confidence limits on parameters of interest derived from the model-particularly the projected mass estimate $M(R<$ $0.4 \mathrm{pc})$.

We begin with the predictions for intrinsic velocity dispersion by substituting the model for $\rho(r)$ into the isotropic Jeans equation. Specifically, from King (1962) we have for the mass density $\rho(r)$ and the surface density (by mass) $\Sigma(R)$ :

$$
\begin{aligned}
z^{2} & \equiv \frac{r_{c}^{2}+r^{2}}{r_{c}^{2}+r_{t}^{2}} \\
\rho(r) & =\frac{K}{\pi r_{c}\left[1+\left(r_{t} / r_{c}\right)^{2}\right]^{3 / 2}} \frac{1}{z^{2}}\left(\frac{1}{z} \arccos z-\sqrt{1-z^{2}}\right) \\
\Sigma(R) & =K\left(\frac{1}{\left[1+\left(R / R_{c}\right)^{2}\right]^{1 / 2}}-\frac{1}{\left[1+\left(R_{t} / R_{c}\right)^{2}\right]^{1 / 2}}\right)^{2},
\end{aligned}
$$

where $r_{c}, R_{c}$ refer to the core radius, $r_{t}, R_{t}$ refer to the tidal radius, and lowercase/uppercase radii denote the radius in three dimensions or projected on the sky, respectively. The Jeans equation takes the form

$$
\begin{aligned}
\sigma_{\text {iso }}^{2}(r) & =\frac{1}{\rho(r)} \int_{r}^{+\infty} G \frac{\rho(r) M(<r)}{r^{2}} d r \\
& =\frac{G}{\rho(r)} \int_{r}^{+\infty} \frac{\rho(r)}{r^{2}} \int_{0}^{r} 4 \pi r^{\prime 2} \rho\left(r^{\prime}\right) d r^{\prime} d r,
\end{aligned}
$$

which is readily evaluated numerically. This model dispersion profile is projected onto the sky using Equations (8) and (10) of LM89 for comparison with observational data.

With this choice of model, three parameters determine the density profile, the dispersion profile and therefore the derived masses of interest: the core radius $r_{c}$, the tidal radius $r_{t}$, and the total cluster mass $M_{\mathrm{cl}}$. The latter parameter is used to normalize the model through the condition

$$
M_{\mathrm{cl}}=4 \pi \int_{0}^{r_{t}} r^{2} \rho(r) d r .
$$




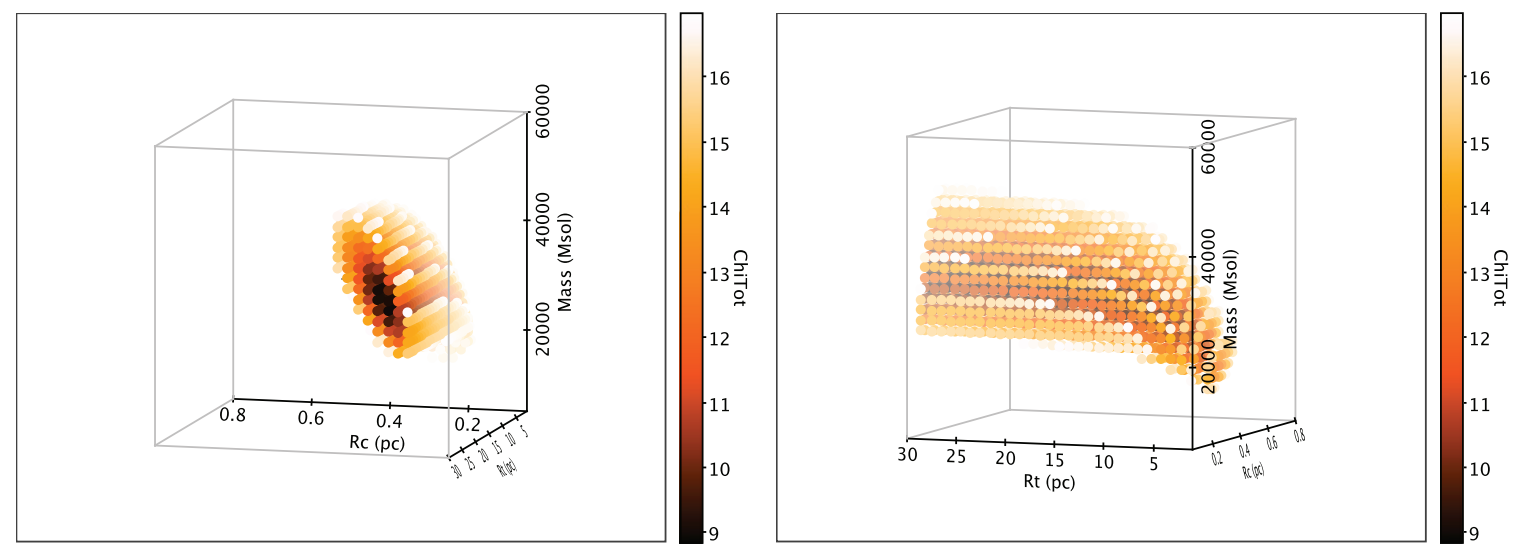

Figure 8. Views of the $\Delta \chi_{\text {full }}^{2}<7.82$ region when both kinematic and surface density data (for stars of mass $10 \leqslant M / M_{\odot} \leqslant 30$; Espinoza et al. 2009) are included in the assessment. Axes are: $R_{c}, R_{t}, M_{\mathrm{cl}}$, with total cluster mass $M_{\mathrm{cl}}$ vertical in each case. Limits shown are: $0.05 \leqslant R_{c} \leqslant 0.8 \mathrm{pc}, 1.0 \leqslant R_{t} \leqslant 30 \mathrm{pc}$, $0.5 \leqslant M_{\mathrm{cl}} \leqslant 6.0 \times 10^{4} M_{\odot}$.

(A color version of this figure is available in the online journal.)

We vary the parameters of the King model (core radius $R_{c}$, tidal radius $R_{t}$, and total cluster mass $M_{\mathrm{cl}}$ ) and map the variation of $\chi^{2}$ when the projected dispersion profile is compared with that obtained from observation. With three model parameters varying we adopt $\Delta \chi^{2}=3.50,7.82$, and 13.93, which correspond to $68 \%, 95 \%$, and $99.7 \%$ of probability (" $1 \sigma$ ", " $2 \sigma$ ", and " $3 \sigma$ ") when three model parameters are allowed to jointly vary (see, for example, Lampton et al. 1976). The limits on quantities derived from these parameters are then given by the range of values of the derived parameters within each $\Delta \chi^{2}$ region of interest.

Our proper motion data do not by themselves constrain the shape of the cluster, as they are concentrated in its innermost regions (for example, our data fall entirely within the estimated $R_{\mathrm{hm}} \approx 0.4 \mathrm{pc}$ of Stolte et al. 2005). We therefore incorporate surface density data $\Sigma_{N}(R)$ (units stars $\mathrm{pc}^{-2}$ ) from the literature. We have proper motion constraints from five radial annuli; comparison of these data alone to the velocity dispersion model yields the figure of merit $\chi_{\text {kinem. }}^{2}$. Comparison of the seven radial estimates of $\Sigma_{N}(R)$ from Espinoza et al. (2009) to model prediction then yields the figure of merit $\chi_{\text {full }}^{2}$. The full figure of merit is then

$$
\begin{aligned}
\chi_{\text {kinem }}^{2} \equiv & \chi_{R}^{2}+\chi_{T}^{2} \\
= & \sum_{i=1}^{5} \frac{\left[\sigma_{R}(\text { data })-\sigma_{R}(\text { model })\right]_{i}^{2}}{\Delta_{R, i}^{2}} \\
& +\sum_{i=1}^{5} \frac{\left[\sigma_{T}(\text { data })-\sigma_{T}(\text { model })\right]_{i}^{2}}{\Delta_{T, i}^{2}} \\
\chi_{\text {full }}^{2} \equiv & \chi_{R}^{2}+\chi_{T}^{2}+\chi_{\Sigma}^{2} \\
= & \chi_{\text {kinem }}^{2}+\sum_{i=1}^{7} \frac{\left[\Sigma_{N}(\text { data })-\Sigma_{N}(\text { model })\right]_{i}^{2}}{\Delta_{\Sigma, i}^{2}}
\end{aligned}
$$

where $\Delta^{2}$ represents the squared errors on each data point.

Since the cluster is in reality mass segregated (e.g., Figer et al. 1999; Stolte et al. 2005), the underlying mass distribution that dominates the velocity dispersion is unlikely to be more centrally concentrated than the massive stars directly amenable to observation. Espinoza et al. (2009) assess $\Sigma_{N}(R)$ for massive stars in different mass ranges; we report here the mass limits using the least centrally concentrated massive-star sample $(10 \leqslant$
$\left.M_{*} / M_{\odot} \leqslant 30\right)$ of Espinoza et al. (2009). Fitted parameters and the behavior of the $\chi^{2}$ surface for different choices of $\Sigma_{N}(R)$ (as well as no constraint on $\Sigma_{N}(R)$, i.e., fitting with kinematic data only) are discussed in Appendix E.

Figure 8 shows the behavior of $\chi^{2}$ as the model parameters $R_{c}, R_{t}, M_{\mathrm{cl}}$ are varied, including the $\Sigma_{N}(R)$ sample just discussed. Figure 9 illustrates the variation of $\chi_{\text {full }}^{2}$ against $R_{c}, R_{t}, M(R<0.4 \mathrm{pc})$. Figure 10 shows radial profiles drawn from within the $\Delta \chi_{\text {full }}^{2}=7.82$ surface in parameter space, which corresponds to $95 \%$ formal significance (or $2 \sigma$ ). As can be seen, a wide range of $M(r<1.0 \mathrm{pc})$ is consistent with the kinematic and surface density data, but a rather narrow range of $M(R<0.4 \mathrm{pc})$ is consistent with the flat plateau and magnitude of the velocity dispersions we measure. Specifically, we find $M(R<0.4 \mathrm{pc})=0.90_{-0.35}^{+0.40} \times 10^{4} M_{\odot}, M(r<1.0 \mathrm{pc})=$ $1.5_{-0.60}^{+0.74} \times 10^{4} M_{\odot}$, and $M_{\mathrm{cl}}=3.16_{-2.09}^{+2.46} \times 10^{4} M_{\odot}$.

All isotropic models tested yield an upper limit on $M(R<$ $0.4 \mathrm{pc}$ ) of $1.30 \times 10^{4} M_{\odot}$ at formal $3 \sigma$ confidence (Appendix E). Inclusion of $\Sigma_{N}(R)$ removes the very low estimates of $M(R<$ $0.4 \mathrm{pc}$ ) from consideration, with the largest lower limit obtained using the full mass range of Espinoza et al. (2009). With the most massive stars included in $\Sigma_{N}(R)$, core radii $R_{c}<0.13 \mathrm{pc}$ are rejected at the $3 \sigma$ level; this level is well above the minimum grid value of $R_{c}=0.05 \mathrm{pc}$. Therefore the grid boundaries are not leading us to assume an artificially compact cluster. The total cluster mass is only very weakly constrained from kinematic data alone.

We also attempted to account for a wide range of cluster anisotropies using the algorithm of LM89. This method differs from the "forward" modeling we describe here, in that the isotropic velocity dispersion profile is modified for anisotropy, the enclosed mass $M(<r)$ estimated from the full Jeans equation using this dispersion profile, and the density profile $\rho(r)$ estimated from the form of $M(<r)$. While we were unsuccessful in reproducing the LM89 approach for a King (1962) profile, parameterization of the cluster with a Plummer profile and allowing for anisotropy (following Leonard et al. 1992) was more fruitful. This yielded a slightly wider range of compatible values of $M(R<0.4 \mathrm{pc})$, though still below $1.5 \times 10^{4} M_{\odot}$ at the formal $2 \sigma$ confidence level. Appendix E outlines the mass modeling using anisotropic cluster models following the method of Leonard et al. (1992); mass limits so produced do not alter the conclusions of this report. 


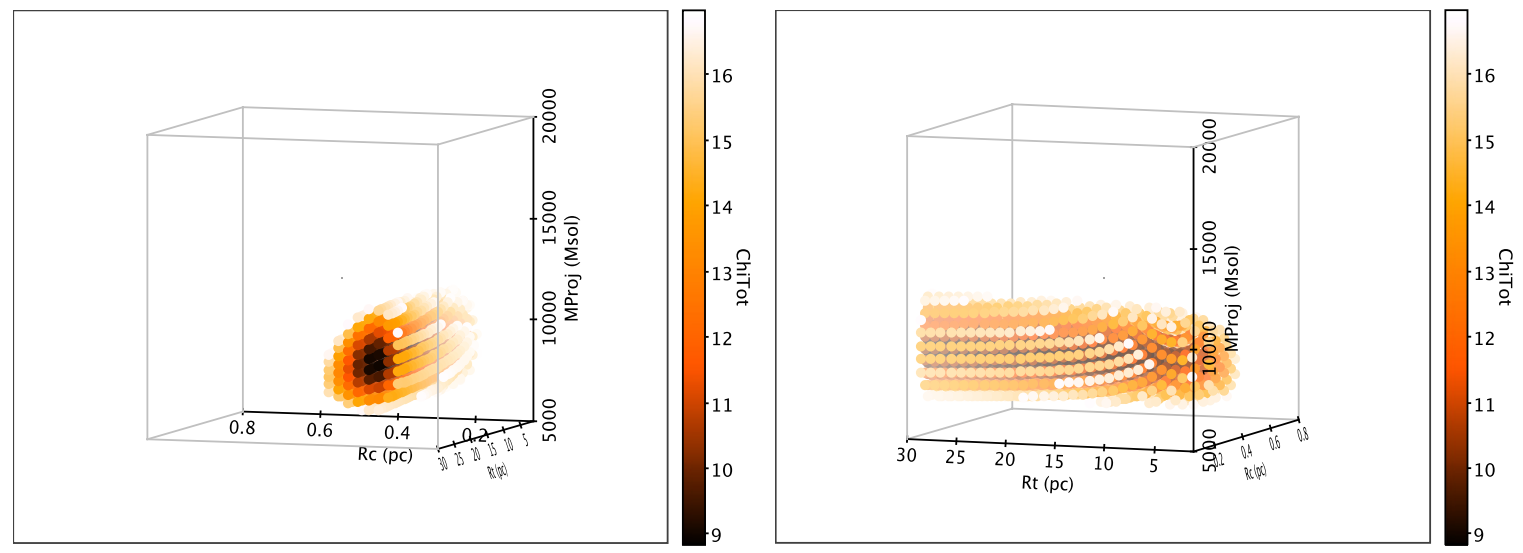

Figure 9. Same as Figure 8, but with $M(R<0.4 \mathrm{pc})$ along the vertical axis. Limits shown are: $0.05 \leqslant R_{c} \leqslant 0.8 \mathrm{pc}, 1.0 \leqslant R_{t} \leqslant 30 \mathrm{pc}, 0.5 \leqslant M(R<0.4 \mathrm{pc}) \leqslant$ $2.0 \times 10^{4} M_{\odot}$.

(A color version of this figure is available in the online journal.)

\section{Isotropic King profiles from 95\% confidence region}
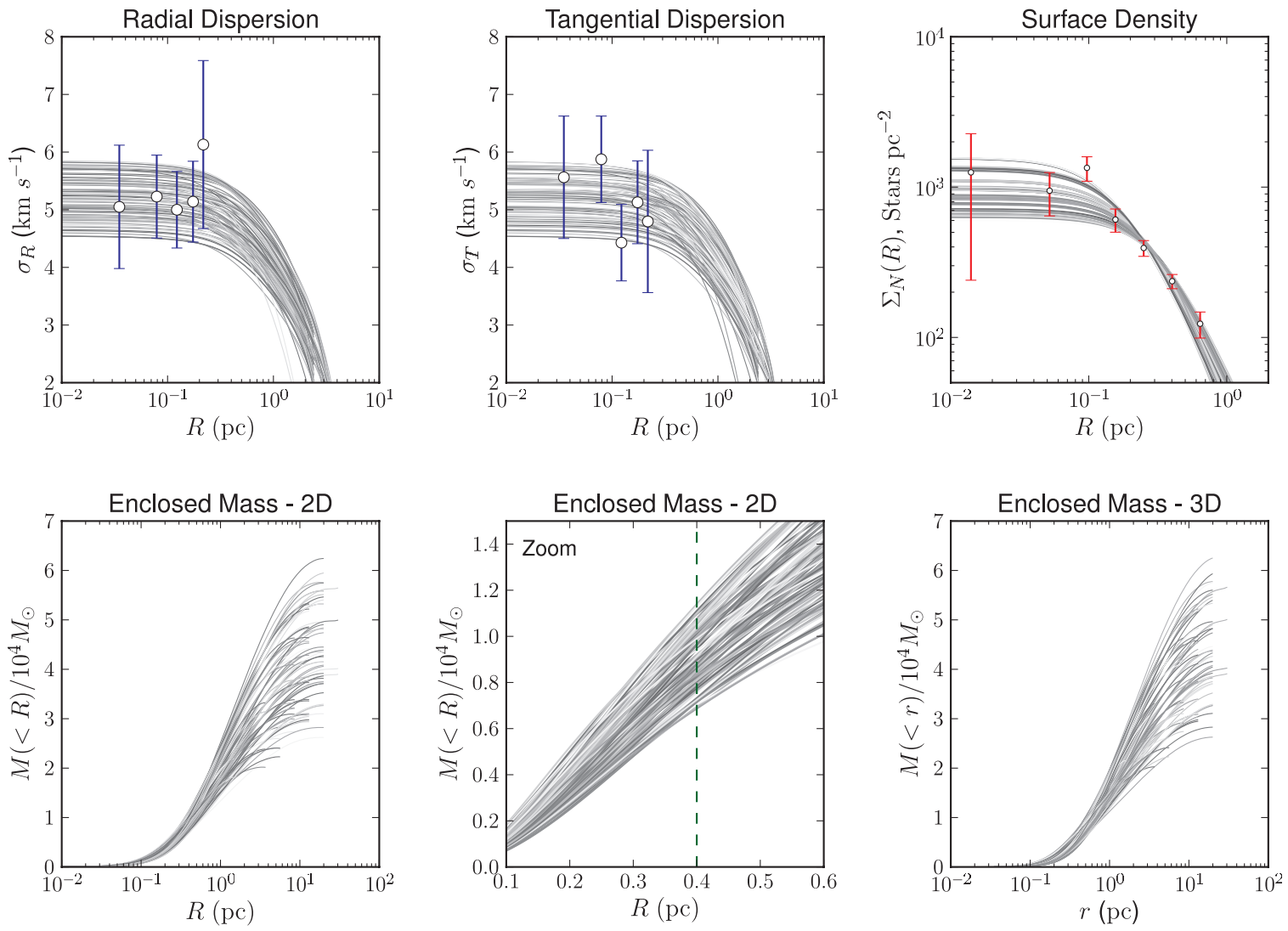

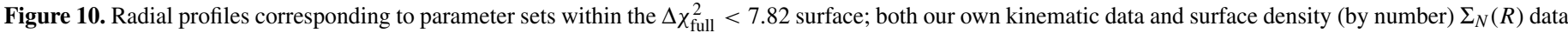

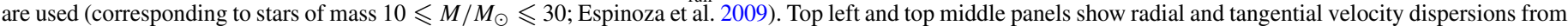

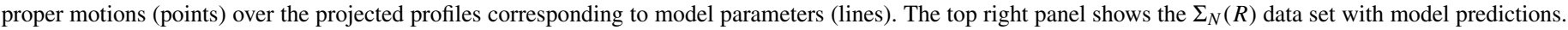

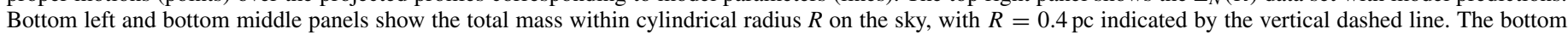
right panel shows the mass enclosed within a sphere of radius $r$ pc from the cluster center. See also Table 10 .

(A color version of this figure is available in the online journal.)

\subsection{Comparison to Literature Mass Estimates}

Unlike photometric mass estimates, which rely heavily on an accurate completeness and extinction correction to map the observed population onto the underlying population, a kinematic mass estimate only requires that the motion of a selection of tracer objects be well measured (and of course that the assumptions in the mass modeling be reasonable). We compare our mass estimates with literature estimates here.

\subsubsection{Literature Mass Estimates}

With the exception of the radial velocity kinematic estimate of Figer et al. (2002), all observational mass estimates of the Arches cluster refer to the projected mass within some radius on the sky, i.e., $M(<R)$. Since this is also the best constrained of our kinematic mass estimates, we focus our literature discussion on these estimates, which are also summarized graphically in Figure 11. 


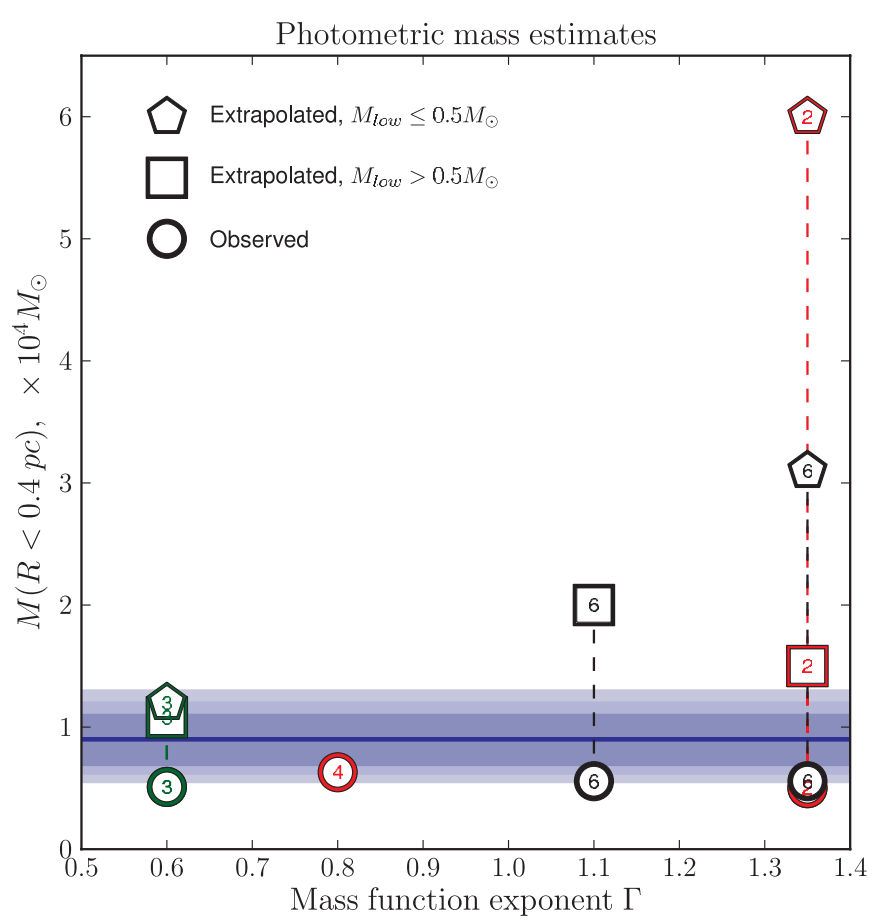

Figure 11. Photometric mass estimates for the Arches cluster from the literature. Symbols give the directly observed photometric mass (circles) and extrapolated mass (where reported; squares and pentagons) depending on the low-mass cutoff assumed. The citation for each estimate is shown inside the symbols (using the numbering of Table 9). The horizontal bands show our model-dependent mass estimate using our dispersion data (at $1 \sigma, 2 \sigma$, and $3 \sigma$ ), and the $\Sigma_{N}(R)$ data set of Espinoza et al. (2009); see Section 5.1 for more detail on the mass modeling used. All masses are reported as $M(R<0.4 \mathrm{pc})$.

(A color version of this figure is available in the online journal.)

Serabyn et al. (1998) extrapolated the mass estimated from observed O-stars down to low masses to estimate the cluster stellar mass; they used $J H K^{\prime}$ imaging with NIRC on Keck-I to estimate a total of $5000 \pm 1000 M_{\odot}$ in $100 \pm 50$ massive O-stars in the cluster, which they extrapolated to the full range of stellar masses using a mass function exponent $\Gamma=1.35$. This yielded $M_{\mathrm{cl}}(R<0.35 \mathrm{pc})=(1.5,6) \times 10^{4} M_{\odot}$, for lower stellar-mass limits $(2,0.1) M_{\odot}$, respectively. ${ }^{16}$

Figer et al. (1999) used NICMOS on the Hubble Space Telescope $(H S T)$ to perform a photometric census down to a photometric limit corresponding to about $6 M_{\odot}$. Within an annulus $0.12 \leqslant R<0.37 \mathrm{pc}$ they measured about $0.51 \times$ $10^{4} M_{\odot}$ in stars, which, using the PDMF measured for the same stars, was extrapolated to a total mass for this outer annulus. The number counts of bright stars were then used to estimate the scale factor from the outer annulus $(0.12 \leqslant R<0.37 \mathrm{pc})$ to the entire inner cluster $(R<0.37 \mathrm{pc})$, yielding a total mass of the inner cluster $M_{\mathrm{cl}}(R<0.37 \mathrm{pc})=(1.08-1.20) \times 10^{4} M_{\odot}$ depending on the lower mass cutoff adopted (1.0-0.1) $M_{\odot}$, and a top-heavy PDMF exponent $(\Gamma \approx 0.6)$.

Stolte et al. (2002) used Gemini NGS/AO photometry and the HST/NICMOS data of Figer et al. (1999) to search for variation of $\Gamma$ with projected radius from the cluster center, using Geneva isochrones (Lejeune \& Schaerer 2001) to convert from magnitude to mass. They obtained a half-mass radius $R_{\mathrm{hm}}=10^{\prime \prime}=0.4 \mathrm{pc}$. By summing the observed mass histogram

\footnotetext{
16 Serabyn et al. (1998) interpret $R=0.35 \mathrm{pc}$ as the total cluster extent, whereas more recent work (e.g., Stolte et al. 2002) suggests a cylinder of this radius contains only about half the total cluster mass. Thus we refer to their estimate as a projected mass estimate.
}

in two projected-radial bins within $R_{\mathrm{hm}}$, they estimated a total mass among the stars measured, of $0.63 \times 10^{4} M_{\odot}$. The authors preferred not to extrapolate the mass function beneath their photometric limit of about $2 M_{\odot}$ due to the uncertainties in so doing. They pointed out also that their estimate is not corrected for incompleteness, and so their estimate for the total mass within $r_{\mathrm{hm}}$ is therefore $M_{\mathrm{cl}}(<0.4 \mathrm{pc}) \sim 10^{4} M_{\odot}$. Note that this is the total stellar mass within a cylinder of radius $R=10^{\prime \prime} \equiv 0.4 \mathrm{pc}$ at $\sim 8 \mathrm{kpc}$, not the total stellar mass in the cluster. Stolte et al. (2002) found a PDMF exponent $\Gamma \sim 0.8 \pm 0.2$ as a spatial average, but with considerable spatial variation as a function of projected radius, though this does not affect their mass estimate. At $R<5^{\prime \prime}$ the PDMF is nearly flat, developing to $\Gamma=1.04 \pm 0.29$ at $5^{\prime \prime}<R<9^{\prime \prime}$ and consistent with Salpeter (1955) at greater radii $(\Gamma=1.69 \pm 0.66$ for $\left.10^{\prime \prime}<R<20^{\prime \prime}\right)$.

Espinoza et al. (2009) report the use of VLT/NACO photometry to fit mass function exponents for two annuli in projected radius; $R<0.2 \mathrm{pc}$ and $0.2<R<0.4 \mathrm{pc}$. Differential reddening corrections on a star-by-star basis were used. The authors prefer to quote the initial masses from Geneva isochrones rather than present-day masses, and therefore give the IMF exponents $\Gamma_{0}$; they find a spatially averaged $\Gamma_{0}=1.1 \pm 0.2$ for $M>10 M_{\odot}$, consistent with Salpeter, and point out that this index is about 0.1-0.15 dex steeper than PDMF indices reported in the literature. Integrating the IMF down to a low-mass cutoff of $1 M_{\odot}$, Espinoza et al. (2009) report $M_{\mathrm{cl}}(R<0.4) \mathrm{pc}=$ $(2 \pm 0.6) \times 10^{4} M_{\odot} \cdot{ }^{17}$ Whether a low-mass truncation exists at all in the Arches mass function is an open question; Espinoza et al. (2009) therefore use a Kroupa (2002) mass function to estimate $M_{\mathrm{cl}}(R<0.4 \mathrm{pc})=(3.1 \pm 0.6) \times 10^{4} M_{\odot}$ without a lower mass cutoff. While the extrapolation to total cluster mass depends on the radial dependence of the PDMF and the density profile, $N$-body models suggest (Harfst et al. 2010) that roughly half the cluster mass is observed between projected radius $R=0.4 \mathrm{pc}$ and the tidal radius $\sim 1 \mathrm{pc}$, which would suggest that the total present-day cluster mass indicated by Espinoza et al. (2009) is closer to $M_{\mathrm{cl}}=(4 \pm 1.2) \times 10^{4} M_{\odot}$ and $M_{\mathrm{cl}}=(6.2 \pm 1.2) \times 10^{4} M_{\odot}$ for lower mass limits of $1.0 M_{\odot}$ and $0.08 M_{\odot}$, respectively.

\subsubsection{Additional Mass Estimates}

In addition to photometric mass estimates discussed above, the Arches total mass is often used as input to models of the formation and evolution of massive clusters. To better place our work in context, all reported mass estimates for the Arches of which we are aware have been collated into Table 9. Most of the total initial mass estimates used in Fokker-Planck (e.g., Kim et al. 1999) and $N$-body simulations (e.g., Kim et al. 2000; Portegies Zwart et al. 2002; Harfst et al. 2010) lie in the range $M_{0} \sim 1-5 \times 10^{4} M_{\odot}$. However, there are some notable outliers; in particular, work approaching the Arches formation from the point of view of cloud fragmentation (generating the IMF) assumes a very high initial cluster mass (e.g., $M_{0} \sim 15 \times 10^{4} M_{\odot}$; Dib 2007). The connection between initial cluster mass for models and observed present-day mass depends on a number of complicated factors that render direct comparison of $M_{0}$ to observation highly assumption dependent;

\footnotetext{
17 Most of the mass loss over the Arches' history is probably dynamical; therefore Espinoza et al. (2009) are really reporting the total initial mass of the stars presently in the cluster, not the initial total cluster stellar mass. This number is closer to the present-day cluster mass $M_{\mathrm{cl}}(<R)$ than $M_{0}(<R)$, so we identify their mass with $M_{\mathrm{cl}}(<R)$ here.
} 
Table 9

All Arches Literature Mass Estimates of which the Authors are Aware

\begin{tabular}{|c|c|c|c|c|c|c|c|c|c|c|}
\hline Ref. & $\begin{array}{c}R_{\text {in }} \\
(\mathrm{pc})\end{array}$ & $\begin{array}{l}R_{\text {out }} \\
(\mathrm{pc})\end{array}$ & $\begin{array}{l}R_{\text {ext }} \\
(\mathrm{pc})\end{array}$ & $\Gamma$ & $\begin{array}{l}M_{\text {low }} \\
\left(M_{\odot}\right)\end{array}$ & $\begin{array}{c}M_{\mathrm{obs}} \\
\left(\times 10^{4} M_{\odot}\right)\end{array}$ & $\begin{array}{c}M_{\text {calc }} \\
\left(\times 10^{4} M_{\odot}\right)\end{array}$ & $\begin{array}{c}\delta\left(M_{\mathrm{obs}}\right) \\
\left(\times 10^{4} M_{\odot}\right)\end{array}$ & $\delta\left(M_{\mathrm{calc}}\right)$ & Notes \\
\hline 1 & $\ldots$ & 1.15 & $\ldots$ & $\ldots$ & $\ldots$ & 0.08 & 0.24 & $\ldots$ & $\ldots$ & Lower limit on total mass \\
\hline 2 & $\ldots$ & 0.35 & $\ldots$ & 1.35 & 2.0 & 0.50 & 1.5 & 0.1 & $\ldots$ & PDMF Salpeter; $M(<R)$ \\
\hline 2 & $\ldots$ & 0.35 & $\ldots$ & 1.35 & 0.1 & 0.50 & 6.0 & 0.1 & $\ldots$ & PDMF Salpeter; $M(<R)$ \\
\hline 3 & 0.12 & 0.35 & $\ldots$ & 0.6 & 1.0 & 0.51 & 1.08 & $\ldots$ & $\ldots$ & PDMF top-heavy; $M(<R)$ \\
\hline 3 & 0.12 & 0.35 & $\ldots$ & 0.6 & 0.1 & 0.51 & 1.20 & $\ldots$ & $\ldots$ & PDMF top-heavy; $M(<R)$ \\
\hline 4 & $\ldots$ & 0.40 & $\ldots$ & $\ldots$ & 2.0 & 0.63 & $\ldots$ & $\ldots$ & $\ldots$ & Rough limit on $M(<R)$ reported \\
\hline 5 & $\ldots$ & 0.23 & $\ldots$ & $\ldots$ & $\ldots$ & 7.0 & $\ldots$ & $\ldots$ & $\ldots$ & Upper limit on $M(<R)$ from radial velocities \\
\hline 6 & $\ldots$ & 0.40 & $\ldots$ & 1.1 & 1.0 & 0.557 & 2.0 & $\ldots$ & 0.6 & low-mass truncation; $M(<R)$ \\
\hline 6 & $\cdots$ & 0.40 & $\cdots$ & 1.35 & 0.08 & 0.557 & 3.1 & $\cdots$ & 0.6 & Kroupa PDMF; no low-mass truncation; $M(<R)$ \\
\hline 7 & $\ldots$ & 2.50 & $\ldots$ & 0.5 & 1.0 & $\ldots$ & 1.60 & $\ldots$ & $\ldots$ & $N$-body; IMF top-heavy; $M_{0}$ reported \\
\hline 7 & $\ldots$ & 2.50 & $\ldots$ & 0.75 & 1.0 & $\ldots$ & 2.00 & $\ldots$ & $\ldots$ & $"$, \\
\hline 7 & $\ldots$ & 2.50 & $\ldots$ & 1.0 & 1.0 & $\ldots$ & 2.80 & $\ldots$ & $\ldots$ & $"$, \\
\hline 8 & $\ldots$ & 1.26 & 3.0 & 2.8 & $\ldots$ & $\ldots$ & 4.00 & $\ldots$ & $\ldots$ & $N$-body; multi-component IMF \\
\hline 9 & $\ldots$ & 0.35 & 2.5 & 0.9 & 1.3 & $\ldots$ & 4.00 & $\ldots$ & $\ldots$ & $N$-body; representative $M_{0}$ reported in figure caption \\
\hline 10 & $\ldots$ & 0.35 & 2.5 & 0.9 & 0.1 & $\ldots$ & 14.50 & $\ldots$ & $\ldots$ & Turbulent-fragmentation calculation \\
\hline 11 & $\ldots$ & 0.35 & 2.4 & 1.1 & 0.9 & $\ldots$ & 5.90 & $\ldots$ & $\ldots$ & $N$-body; observations reported in Kim et al. (2006) \\
\hline 12 & $\ldots$ & 0.40 & $\ldots$ & 1.35 & 0.5 & $\ldots$ & 1.80 & $\ldots$ & $\ldots$ & $N$-body, Salpeter IMF; present-day $M(R<0.4 \mathrm{pc})$ \\
\hline 12 & $\ldots$ & 0.40 & 1.0 & 1.35 & 0.5 & $\ldots$ & 3.60 & $\ldots$ & $\ldots$ & Present-day simulated mass within projected radius $R=1.0 \mathrm{pc}$ \\
\hline 12 & $\ldots$ & 0.40 & 2.8 & 1.35 & 0.5 & $\ldots$ & 4.90 & $\ldots$ & 0.8 & Total initial cluster mass $M_{0}$ with low-mass truncation \\
\hline 12 & $\ldots$ & 0.40 & 2.8 & 1.35 & 1.0 & $\ldots$ & 3.60 & $\ldots$ & 0.6 & Total initial cluster mass $M_{0}$ with moderate-mass truncation \\
\hline 12 & $\ldots$ & 0.40 & 2.8 & 1.35 & 4.0 & $\ldots$ & 1.90 & $\ldots$ & 0.3 & Total initial cluster mass $M_{0}$ with moderate-mass truncation \\
\hline
\end{tabular}

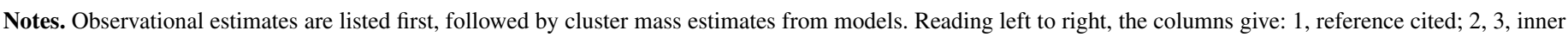

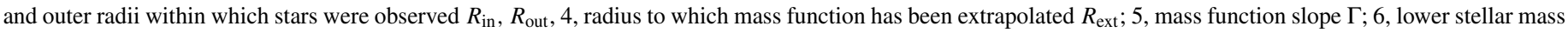

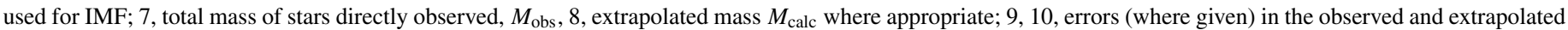

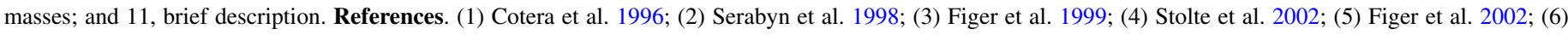
Espinoza et al. 2009; (7) Kim et al. 2000; (8) Portegies Zwart et al. 2002; (9) Kim et al. 2006; (10) Dib 2007; (11) Chatterjee et al. 2009; (12) Harfst et al. 2010.

the Arches has probably lost roughly half of its stellar mass since formation (e.g., Kim et al. 2000; Harfst et al. 2010).

\subsubsection{Proper Motion Derived Mass Estimate Compared to Literature Mass Estimates}

Isotropic (King 1962) profiles produce estimates of the present-day projected mass $M(R<0.4 \mathrm{pc})$ that are at least $3 \sigma$ below the $M(R<0.4 \mathrm{pc})=(3.1 \pm 0.6) \times 10^{4} M_{\odot}$ derived by Espinoza et al. (2009) under the assumption of a non-topheavy mass function and no lower-mass truncation. In contrast, photometric estimates assuming either a low-mass truncation or top-heavy mass function, or both, are more compatible with our dispersion data under the assumptions of our mass models (Figure 11). In particular, our upper limit of $1.30 \times 10^{4} M_{\odot}$ from isotropic King modeling is $1.5 \sigma$ below the photometric estimate of Espinoza et al. (2009) with a lower mass cutoff at $1 M_{\odot}$ using a mass function that is not strongly top-heavy. In addition, the mass ranges of Figer et al. (1999) are highly compatible with our dispersion-based mass estimate.

Within the limitations of our modeling, then, our velocity dispersion estimate is compatible with a mass function that is either top-heavy, truncated at low mass, or both. A Salpeter PDMF without low-mass truncation is not indicated by our data.

We remind the reader that our mass limit is a first estimate with a straightforward model, which is likely subject to update when the full machinery of simulation is brought to bear on the problem using our dispersions as a constraint. On the modeling side, several factors complicate the interpretation of velocity dispersion data. First, the location of the Arches cluster in a strong tidal field suggests the assumptions of spherical symmetry and negligible rotation may be violated. Second, while the degree of mass segregation in a young post-collapse cluster is different for stars of different masses, after $\sim 2 \mathrm{Myr}$, the massive stars whose motion we measure $\left(M \gtrsim 10 M_{\odot}\right)$ are likely to have undergone some mass segregation (Allison et al. 2009). Therefore we are measuring tracer stars whose velocity dispersion may be biased to low values. Velocity dispersions constructed from proper motion observations of stars solely with masses $M \lesssim 10 M_{\odot}$ (i.e., $K^{\prime} \gtrsim 16$ ) would provide a second mass estimate from a population less sensitive to mass segregation (see Figure 2 of Allison et al. 2009). For the present data set this sample is almost entirely on the steeply rising part of the error-magnitude curve (Figure 2). Investigation of this sample is outside the scope of the present paper. At the present stage of our investigations, we limit ourselves to pointing out that our mass estimate may be biased to low values by our sample selection of massive stars that have likely undergone some degree of dynamical mass segregation.

On the observational side, we do not yet have sufficiently precise motions outside $0.2 \mathrm{pc}$ to constrain $M(R)$ outside this region, and have had to resort to projection of models that have significant caveats when applied to this cluster. Future observations of the outer fields should remedy this situation.

Note that, unlike with radial velocity studies, binaries are unlikely to have an effect on the velocity dispersion measurement we report here. To produce an effect of the same order of magnitude as the proper motion dispersion we obtain, a substantial binary population would be needed in which the binary orbit shifted the center of near-IR light by $\sim 0.3$ mas over the three-year time base of our observations. A binary with components 150 and $50 M_{\odot}$ in a 1000 day orbit would exhibit semimajor axis and orbital speed of adequate magnitude ( $a \simeq 0.36$ mas, $v \simeq 0.13 \mathrm{mas} \mathrm{yr}^{-1}$, respectively) to produce this effect (assuming the near-IR brightness ratio corresponding to this mass ratio 
is sufficient for the center of light to move). However, we expect such systems to be too rare to produce any effect on the dispersion measurement over the Arches population. Furthermore, such systems would be confined to the brightest magnitude bin in our analysis, leading to a decrease in measured dispersion with increasing apparent magnitude, which is not observed. Appealing to high eccentricity introduces an additional selection effect (on the orientation of the orbit to produce measurable motion). Thus we conclude binaries are an insignificant contributor to the measured velocity dispersion in the Arches cluster (compare with Gieles et al. 2010).

\subsection{Mean Motion of the Cluster}

The 2D Gaussian profile of the field component in the VPD shows an axis ratio that is roughly constant with magnitude, while its contribution to the sample in the field of view increases as fainter objects are probed. Its orientation in the VPD is consistent with the Galactic plane, indicating that the velocity dispersion along the field major axis in the VPD is strongly affected by differential rotation.

The bulk motion of the Arches with respect to the field population is $172 \pm 15 \mathrm{~km} \mathrm{~s}^{-1}$ (the inverse variance-weighted average of the $14<K<16$ and $16<K<18$ bins). Including the $18<K<20$ bin revises this figure downward to $153 \pm$ $11 \mathrm{~km} \mathrm{~s}^{-1}$; however, in this magnitude range the proper motion error curve rises steeply with magnitude (Figure 5), so objects this faint may be particularly vulnerable to misclassification bias (Appendix D). This is slightly smaller than the $212 \pm 29 \mathrm{~km} \mathrm{~s}^{-1}$ determined previously (Stolte et al. 2008). This is probably due to two competing biases in the previous work that oppose each other: Stolte et al. (2008) imposed a hard membership limit, where all objects within a certain velocity from the cluster center in the VPD are denoted cluster objects, which tends to exaggerate the cluster-field separation in the VPD by cutting off one side of the field component. Conversely, they included objects at all magnitudes in their estimate of the bulk motion, which tends to reduce the estimated component separation (see Appendix D).

In their study of the Arches bulk motion, Stolte et al. (2008) found that, although the Arches is unlikely to be on a circular orbit, integration of its path through the potential of the inner Milky Way indicated the cluster was unlikely to pass sufficiently close to the GC to spiral in toward it and donate its stars to the GC nuclear cluster. Our revised motion estimate makes the Arches orbit slightly more compatible with circular motion. Following the arguments of Stolte et al. (2008), if on a circular orbit, $\mathbf{v . r}=0$ then demands an enclosed mass only $1.5 \sigma$ above that measured photometrically (Launhardt et al. 2002). At first glance, our new bulk motion supports the conclusions of Stolte et al. (2008) that a cluster that is dynamically similar to the Arches is unlikely to be a future source of young stars for the GC star cluster. Integration of the Arches motion through the potential of the inner Milky Way using our new motion determination is required to draw further conclusions about the formation and subsequent motion of the cluster.

Interpretation of the cluster bulk motion is complicated by three factors. First, the kinematic parameters of the field component depend on the distribution of tracer stars along the line of sight as well as their motion. Differential rotation by field stars participating in Galactic rotation may therefore vary with tracer-star brightness (with observations to different depths picking up different field-tracer populations). Second, the field population (or a significant component of the field population) may show its own motion beyond Galactic rotation; for example this motion may be dominated by bar rotation on the nearside of the GC (if the far side of the bar suffers from higher extinction). Or, rotation of the NSD could impose a mean motion of the field component with respect to the Arches cluster. Third, extinction variations along the line of sight coupled with the low-number statistics $(\sim$ few tens of field objects in each magnitude bin; Table 6) may reduce the validity of a Gaussian to represent the field component in the first place. Thus, our quoted error of $15 \mathrm{~km} \mathrm{~s}^{-1}$ on the Arches bulk motion is likely an underestimate. Stolte et al. (2008) discuss further some of the difficulties associated with interpreting a bulk motion against a mean-field population.

\subsection{Properties of the Field Population}

Within the measurement errors, the orientation of the field ellipse is entirely consistent with the direction of the vector joining the cluster and field centers in the VPD, indicating the Arches moves along the direction of preferential motion for the field (Table 6).

To our knowledge, the covariance $\mathbf{Z}_{k}$ of the field component provides the first estimate of the stellar velocity dispersion of the bulge along such a close sight line to the GC. This will allow a direct constraint on the bulge potential along this sight line, which itself is a key ingredient in the use of cluster bulk motion to assess its likely path through the inner Milky Way (Stolte et al. 2008). Here we restrict ourselves to a comparison of $\mathbf{Z}_{k}$ with the velocity dispersion of the bulge at higher latitudes.

The bulge is a highly complex stellar structure, with many basic parameters presently under debate, complicated by shifting nomenclature in the literature. Several components appear to be present, with the relationship between them still far from settled. Chemical evidence suggests most of the stars formed early and rapidly, as might be expected for a "classical" bulge component (e.g., McWilliam \& Rich 1994; Lecureur et al. 2007), while the spatial arrangement and motion of the stars suggest a bar structure, driving a "boxy/peanut" bulge (e.g., Dwek et al. 1995; Howard et al. 2009; McWilliam \& Zoccali 2010; Shen et al. 2010).

Whatever its formation history, the present-day bulge shows variation of stellar kinematics with metallicity. Soto et al. (2007) report variation of the shape of the $\sigma_{r}, \sigma_{l}$ velocity ellipsoid with metallicity (Zhao et al. 1994 provide an early detection of vertex deviation in the bulge). Babusiaux et al. (2010) present proper motion dispersions as a function of metallicity for the Baade's window (hereafter $\mathrm{BW}$ ) field $\left(l=0^{\circ}, b=-0.4\right)$, significantly farther out from the GC than our sample. For stars of approximately solar and higher metallicity, they report $\sigma_{l}, \sigma_{b}=107 \pm 6,94 \pm 6 \mathrm{~km} \mathrm{~s}^{-1}$. For stars with $[\mathrm{Fe} / \mathrm{H}]<$ -0.14 , Babusiaux et al. (2010) report $\sigma_{l}, \sigma_{b}=138 \pm 12,103 \pm$ $7 \mathrm{~km} \mathrm{~s}^{-1}$.

To estimate the velocity dispersion of the Arches field component we take the variance-weighted mean of the determinations from the three non-overlapping bins $\left(14.0 \leqslant K^{\prime}<16.0\right.$, $16.0 \leqslant K^{\prime}<18.0$, and $18.0 \leqslant K^{\prime}<20.0$ ). This yields majoraxis and minor-axis dispersions of $\overline{\sigma_{a, f}}=2.72 \pm 0.15$ mas yr$^{-1}$ and $\overline{\sigma_{b, f}}=1.69 \pm 0.10 \mathrm{mas} \mathrm{yr}^{-1}$, which scale to the distance of the GC ( $8.4 \pm 0.4 \mathrm{kpc}$; Ghez et al. 2008) as $103 \pm 7.7 \mathrm{~km} \mathrm{~s}^{-1}$ and $64 \pm 5.0 \mathrm{~km} \mathrm{~s}^{-1}$. The orientation of the field component in the VPD, expressed as a position angle east of north, is $\overline{\theta_{f}}=+30.5 \pm 7.1$ (motion along the Galactic plane corresponds to about +27.1 in the VPD). For the remainder of this section we therefore identify the field velocity dispersion major axis 
with the Galactic plane, so $\sigma_{a, f}, \sigma_{b, f}$ represent the field velocity dispersion in Galactic longitude and latitude, respectively. Thus our field velocity dispersion is more consistent with the higher-metallicity BW stars, which is currently interpreted by Babusiaux et al. (2010) as a bar-dominated population.

Some caution is warranted interpreting $\mathbf{Z}_{k}$ for the field, as a number of parameters of the field population are still not fully understood. First, the NSD imprints its own motion on the field, which may be coherent and different from the motion of the bulge/bar system. Second, the mass distribution of the composite bulge along our line of sight is not fully constrained; for example, estimates of bar orientation within the Galactic plane still range by $45^{\circ}$ (e.g., Picaud \& Robin 2004; Benjamin et al. 2005; Robin et al. 2009; McWilliam \& Zoccali 2010). Third, astrometric completeness likely biases the bulge motion we observe toward the nearside of the GC, so the field motion is sampled at some preferred mean distance from the GC. Fourth, the mixture of bulge and bar components making up the field population is not yet fully constrained.

\subsection{Membership Probabilities and $L^{\prime}$-excess Sources}

Our refined membership probabilities allow a clean-cluster sample to be extracted for further work. Table 5 lists the formal membership probabilities (Equation (2)) for well-measured stars in our sample. The use of this cleaned sample to probe the cluster mass function is underway by our group (McCrady et al. 2011), and will be reported in a following paper. For now, we note that the Keck-Keck motions provide support to the conclusions of Stolte et al. (2010), that a significant number of stars with circumstellar disks are likely present in the Arches cluster. These stars appear redward of the locus of most stars in the Arches in the $H-K \mathrm{CMD}$; since $L^{\prime}$-excess sources cannot be distinguished from field stars based on $H-K^{\prime}$ color alone, kinematic separation is essential. Of 16 objects falling within the locus of points redward of the cluster main sequence within the CMD (Figure 7), 6 show proper motions suggestive of cluster membership. More generally, however, we find that most of the outliers from the main locus of Arches stars in the $H-K C M D$ are indeed kinematically associated with the field.

\section{CONCLUSIONS}

With uniform observational setup over a sufficient time baseline and careful accounting for a number of sources of proper motion error, we have measured the internal velocity dispersion of the Arches cluster for the first time, finding $\sigma=$ $0.15 \pm 0.01 \mathrm{mas} \mathrm{yr}^{-1}$, which corresponds to $5.4 \pm 0.4 \mathrm{~km} \mathrm{~s}^{-1}$ at a distance of $8.4 \mathrm{kpc}$.

We have used this dispersion to test the photometric estimates of the PDMF of the Arches cluster. The total mass is likely in the range $M(r<1.0 \mathrm{pc})=1.5_{-0.60}^{+0.74} \times 10^{4} M_{\odot}$, but this is only weakly constrained by kinematic data and is consistent with nearly all suggestions of total cluster mass from the modeling literature. The projected mass (i.e., mass contained within a cylinder of radius $R$ on the sky) is rather better constrained; we find $M(R<0.4 \mathrm{pc})=0.90_{-0.35}^{+0.40} \times 10^{4} M_{\odot}$ at formal $3 \sigma$ confidence. The upper bound of this range is $3 \sigma$ below the photometric estimate for $M(R<0.4 \mathrm{pc})$ estimated by Espinoza et al. (2009) under the assumptions of a non-top-heavy mass function without low-mass truncation. If a substantial contribution from massive binaries were unknowingly included in our measurement, this would strengthen our conclusion because the upper cluster mass bound would accordingly be reduced. This is the first mass estimate for the Arches based on proper motion velocity dispersion.

We have also revised the bulk motion of the Arches slightly downward. Our updated motion of $172 \pm 15 \mathrm{~km} \mathrm{~s}^{-1}$ is only slightly lower than the $212 \pm 29 \mathrm{~km} \mathrm{~s}^{-1}$ determined previously (Stolte et al. 2008). Taken at face value, this supports the previous conclusion that the Arches cluster is unlikely to pass within 10 pc of the GC.

Finally, we have provided the first estimate (to our knowledge) of the velocity dispersion of the bulge along such a close sight line to the GC; this estimate is $(103,64) \pm(7.7,5.0) \mathrm{km} \mathrm{s}^{-1}$, with the major axis coincident with the Galactic plane, to within the uncertainties.

Support for this work was provided by NSF grants AST 0406816 and AST 09-09218, and the NSF Science and Technology Center for Adaptive Optics, managed by the University of California, Santa Cruz (AST 98-76783), and the Levine-Leichtman Family Foundation. A.S. is supported by a DFG Emmy Noether grant under ID STO 469/3-1. The W. M. Keck Observatory is operated as a scientific partnership among the California Institute of Technology, the University of California and the National Aeronautics and Space Administration. The Observatory was made possible by the generous financial support of the W. M. Keck Foundation. The authors wish to recognize and acknowledge the very significant cultural role and reverence that the summit of Mauna Kea has always had within the indigenous Hawaiian community. We are most fortunate to have the opportunity to conduct observations from this mountain. The authors thank Sungsoo Kim for helpful discussion and for bringing a very useful paper to our attention. We also thank the anonymous referee for insightful comments which clarified the presentation of some of the points in this paper.

\section{APPENDIX A}

\section{PRODUCTION OF MOTIONS FROM STAR LISTS}

Here we provide details of the procedures used to produce proper motions from the star lists. The steps are as follows.

1. Choice of initial reference stars, and initial mapping onto $t_{0}$. An initial list was constructed of 11 bright stars that were well measured in all epochs and cover the full area of the detector, and used for the initial registration of each epoch onto $t_{0}=2008.5$. This epoch was chosen because its star list is of high quality (Table 1) and the epoch itself will be near the pivot point of the straight-line fits to the positional time series for most of the stars. A six-term linear transformation was used for this mapping for each epoch, accounting for positional shift, global scaling, rotation, a difference in scale factors in $X$ and $Y$ ("on-axis skew"), and deviation from $90^{\circ}$ of the angle between axes ("off-axis skew").

2. Matching of all stars within $t_{0}$. Using this initial mapping, all stars were matched to their counterparts in $t_{0}$ (where present) by proximity in $t_{0}$ and magnitude. A matching radius of 5 pixels (approximately the PSF core FWHM) and a broad magnitude tolerance of 3 mag were used. This yields positional differences between predicted and observed positions (hereafter "deltas") in $t_{0}$ for matched pairs using the first-guess transformation.

3. Fitting of reference frame mapping for matching. The previous step typically produces deltas for $\sim 300$ stars at 


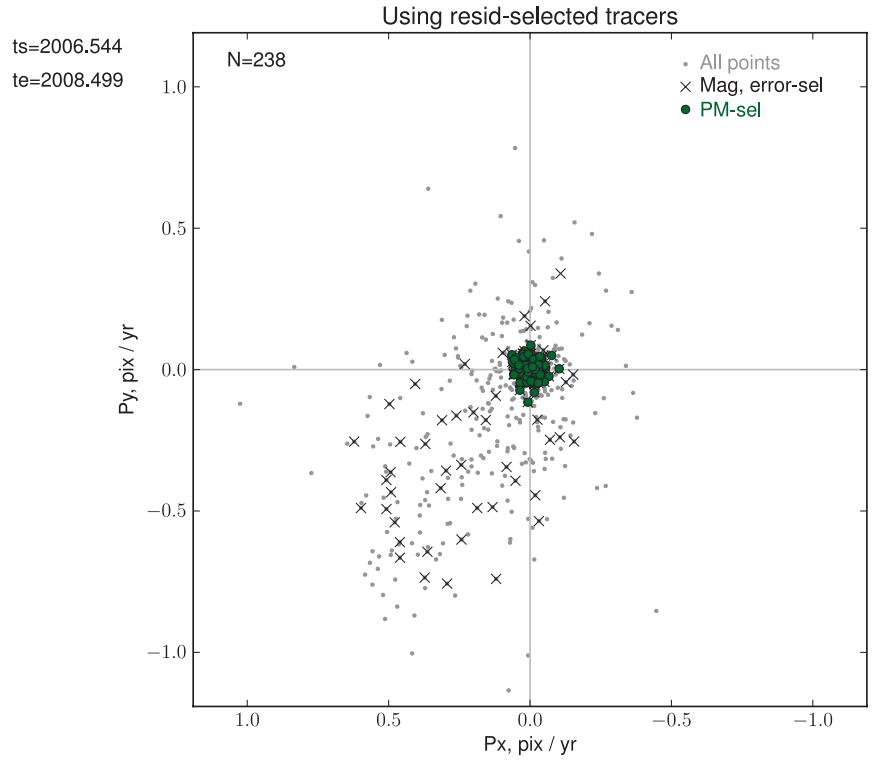

Figure 12. Culling of reference stars during the frame-mapping process, in this case mapping epoch 2006.54 onto the frame of epoch $t_{0}=2008.50$. Yellow points: all matches. Black points: selected for magnitude $\left(K^{\prime} \leqslant 17.5\right)$. Green points: reference stars selected by position residual from the center of mass of the magnitude-selected sample and with outliers clipped during the mapping (in this plot the motions have been shifted to the center of mass of the selected objects).

(A color version of this figure is available in the online journal.)

$K^{\prime}<17.5$ across each pair of epochs $\left(t-t_{0}\right)$. The field population displays significant motion dispersion in a preferential direction close to the Galactic plane. Field objects must therefore be removed from the sample of reference stars to avoid biasing the offsets and magnification factors when mapping the reference frames. From the positional deltas of reference stars, the center of the distribution in the VPD is estimated and the standard deviation of motions in each direction from this center of mass estimated. Objects farther than $2 \sigma$ from this estimated center of mass are removed. This process is repeated twice to produce a cleaned list of reference stars; typically 260 objects survive this process. These objects are used to re-map the epochs onto $t_{0}$ using a full six-term linear transformation. Clipping of outliers in this epoch mapping typically removes a further 30 stars in each epoch (Figure 12).

4. Trim coincident close pairs of stars. At this stage we have the master list of measurements of each object, in the frame in which the object was originally measured. To mitigate confusion by known objects as much as possible, all coincident pairs with separation $<75$ mas are removed from consideration for each epoch. This typically removes 20-40 objects from the position lists at each epoch (column

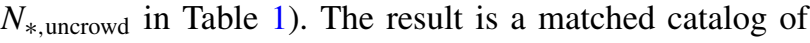
1114 objects present in at least two epochs.

5. Reference frame mapping for motions. Armed with the matched list of objects and their measurements at each epoch, likely cluster members (at $K<17.5$ ) are used as reference stars to map each epoch onto $t_{0}$, using the same weighting and clipping as step 3. We find (Appendix C) that a second-order transformation in $X$ and $Y$ is sufficient to capture most of the residual higher-order effective distortions between epochs without falling prey to overfitting of few stars with a high-order transformation.
6. Motion extraction in $t_{0}$. A first pass at stellar motions is estimated by fitting a linear trend to the positional time series $x(t), y(t)$ of each star in the reference frame $t_{0}$. For each star, the weighted mean time $\bar{t}=\sum_{i} t_{i} w_{i} / \sum_{i} w_{i}$ is evaluated so that the fit becomes $x(t)=a+b(t-\bar{t})$. Weights $w_{i}=1 / \sigma_{i}^{2}$ are the inverse of the variance of each measurement due to positional uncertainty. This removes correlation between errors in the parameters (e.g., Press et al. 2002); the center of mass of the data is first determined then the slope pivoted about this point to find the best-fit positional gradient. The proper motion error is the formal error on the best-fit slope: $\sigma_{b}^{2}=1 /\left(\sum_{i=1}^{N}(t-\bar{t})^{2} / \sigma_{i}^{2}\right)$. To mitigate sensitivity to short-term excursions in position, for objects measured in $\geqslant 4$ epochs, two passes of sigmaclipping at $3 \sigma$ are applied. Note that $\bar{t}$ is a property of $\sigma_{i}(t)$ and thus is estimated separately for each star and for each coordinate. Motions are estimated for all 805 stars with $\geqslant 3$ epochs of measurement.

7. Refinement of the reference position list. When choosing a reference frame in which to evaluate motions, our goal is a reference list onto which cluster members can be mapped with as little scatter as possible due to measurement and fitting error. We generate a reference frame by evaluating at some time $t_{\text {ref }}$ the straight-line fits to the positional time series of cluster reference stars. Positional errors in this predicted frame ("predictive errors") are evaluated by propagating the errors on the fit coefficients $a, b$ for each star. By choosing $t_{\text {ref }}$ to be near the pivot point $\bar{t}$ of the greatest number of reference stars, we aim to minimize the error of the predicted positions in the constructed reference frame. The distribution of $\bar{t}$ is nearly Gaussian with $\bar{t}=2008.0 \pm 0.4(1 \sigma)$; we therefore adopt $t_{\text {ref }}$ $=2008.0$ to evaluate the reference epoch. To evaluate the degree to which this mean reference frame improves the mapping, motions and their errors for each star were evaluated using the quad sum (centroiding + alignment) errors when mapped to $t_{0}$ and (centroiding + alignment + predictive) when mapped to $t_{\text {ref }}$. Motion errors are improved by up to $20 \%$ for some bright objects, with median improvement up to $4 \%$ for well-measured objects (Figure 13).

8. Re-mapping and re-extraction of motions. Finally, the star list from each epoch is mapped onto the constructed reference frame $t_{\text {ref }}$ and motions in this frame evaluated in the manner of step 7 . Table 3 gives the fitted parameters and the number of reference stars used in the mapping from each epoch to the $t_{\text {ref }}$ frame. Provided the motions of cluster stars in the field of view do not themselves describe a secondorder or lower transformation (e.g., rotation or contraction of the cluster) to within our ability to determine, then the parameters taking reference frame 2008.50 to $\bar{t}$ should be consistent with zero, as is observed (Table 3 ). We find that, when applied to stars near the edges of the detector, the size of the positional shifts due to the quadratic terms in the mapping are one to a few times the size of the centroiding error for bright $\left(K^{\prime}<16\right)$ objects (Table 3$)$.

9. Evaluation and incorporation of additional error sources. The distribution of fits to the velocities thus produced was examined for additional sources of random error. It became readily apparent that a significant source of error along the time series was not taken into account by the steps above. When characterized (Section 3.3.3), steps 6-9 were repeated with this error term included. 

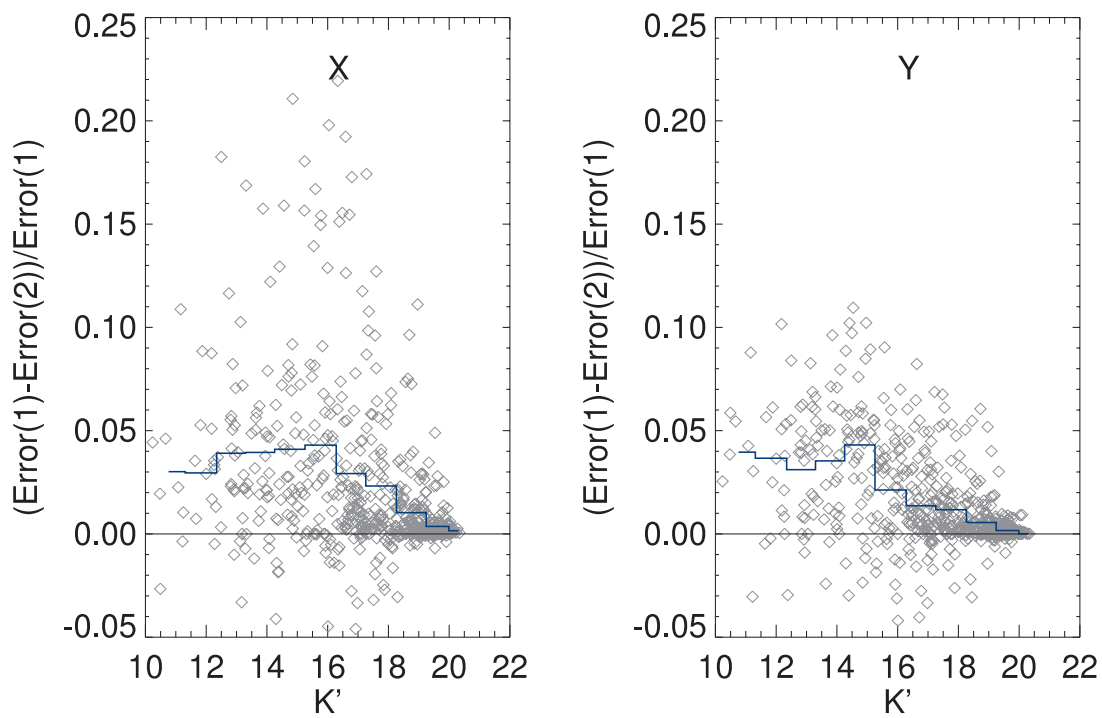

Figure 13. Reduction in velocity error when frames are mapped onto a reference list constructed from a first pass at proper motion fitting (2), over frame mapping onto a single star list at epoch $t_{0}$ (1). The change in error is expressed as the ratio of the difference between (1) and (2) to the original error (1). The blue line reports the median improvement within each magnitude bin. Reading from left to right, panels indicate errors along $X$ and $Y$.

(A color version of this figure is available in the online journal.)
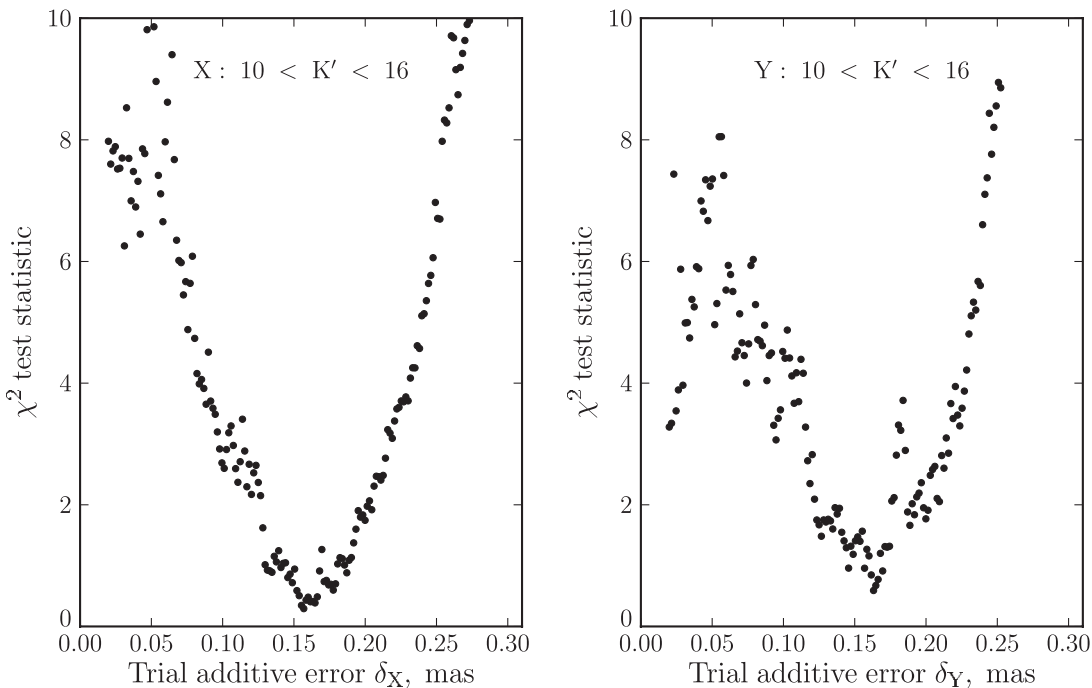

Figure 14. Determination of additive errors $\delta_{x}$ (left) and $\delta_{y}$ (right), for objects in the brightness range $\left(10.0 \leqslant K^{\prime} \leqslant 16\right.$.). For each trial additive error, the distribution of $\chi^{2}$ values from the velocity fits to each star is compared to that expected under statistical error, using the $\chi^{2}$ test. The statistic of this comparison is plotted here.

\section{APPENDIX B}

\section{ADDITIONAL ERRORS BEYOND CENTROIDING AND ALIGNMENT ERRORS}

\section{B.1. Estimating the Magnitude of Additive Random Errors}

When frames are mapped together and motions estimated using the quadrature sum of the centroiding and additive errors, the resulting distribution of $\chi_{v}^{2}$ from the velocity fits is significantly different from the canonical $\chi^{2}$ distribution, indicating that the random errors characterized in Sections 3.3.1 and 3.3.2 are not sufficient to account for the random variation about the best fit actually observed. We detail here the estimate of the "additive" error $\delta_{x}, \delta_{y}$ that must be added in quadrature to rectify this situation.

Once stars are aligned into the $t_{\text {ref }}$ reference frame, velocities are re-fit for trial values of $\delta_{x}, \delta_{y}$, and the resulting distribution of $\chi^{2}$ values from the velocity fits are compared to expectations.
Two tests were evaluated to make the comparison. First, the $\chi^{2}$ test was evaluated for the difference between the $\chi^{2}$ histogram and the theoretical expectation at each trial additive error. Because of the binning required, this statistic does not vary smoothly with the trial error; to estimate the minimum, a secondorder polynomial was fit to the trough in the fit statistic. This yields estimates for the best-fit additive error in each coordinate (denoted $\overline{\delta_{x}}, \overline{\delta_{y}}$ ). Second, the two-sided Kolmogorov-Smirnov test was used as a fit statistic to obviate the need for binning. While the minima returned by the two measures are broadly consistent with each other, we adopt the $\chi^{2}$ test since it appears to provide a more sensitive determination of the bestfit additive error (Figure 14). Errors on this determination of $\overline{\delta_{x}} \& \overline{\delta_{y}}$ are estimated by simulation; sets of positional time series are constructed under Gaussian noise with amplitudes as in the real data and perturbed by additional spatially uniform error $\delta_{x, \text { in }}, \delta_{y, \text { in }}$ (while keeping the error used to re-determine the additive errors as the quadrature sum of alignment and 


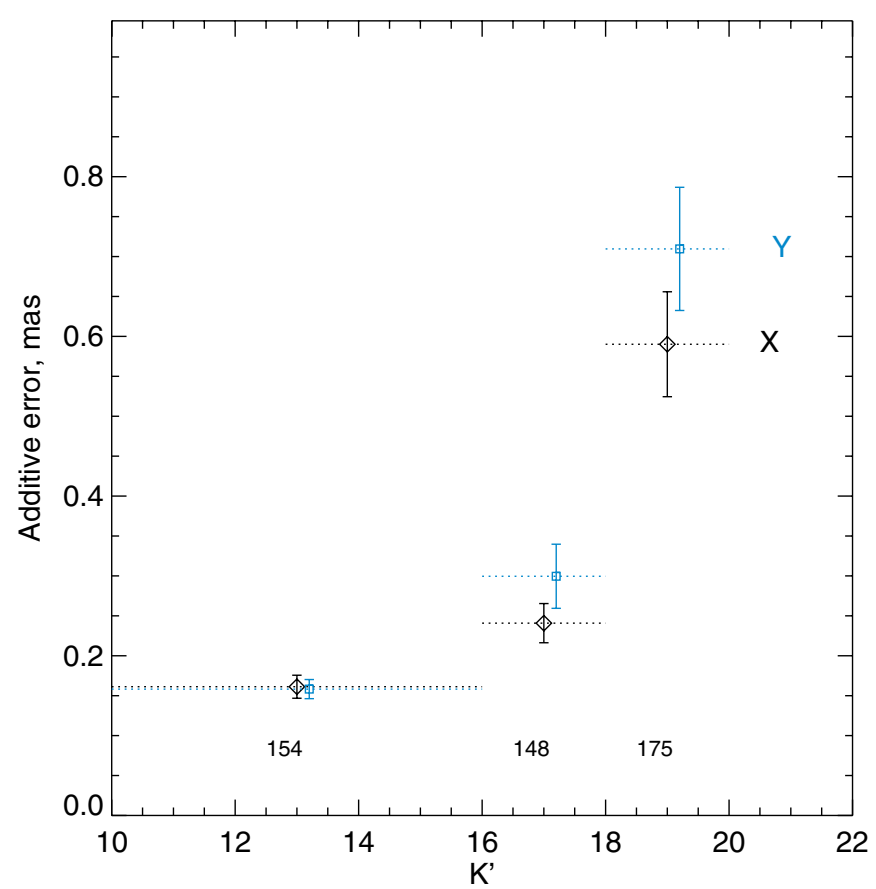

Figure 15. Additive error as a function of magnitude. Black diamonds: X; blue squares: Y, offset along the horizontal axis slightly for clarity. The sample size in each magnitude bin are indicated within the frame. Horizontal dashed lines indicate the magnitude ranges in each bin.

(A color version of this figure is available in the online journal.) centroiding errors). The rms of $\left(\delta_{x, \text { in }}-\overline{\delta_{x}}\right),\left(\delta_{y, \text { in }}-\overline{\delta_{y}}\right)$ is then adopted as the error in the additive errors. Only stars with five measurements are used to estimate $\delta_{x}$ and $\delta_{y}$.

As the balance of dominant error terms evolves with magnitude (e.g., Fritz et al. 2010), we might expect $\bar{\delta}$ to also vary with magnitude. We therefore break the sample into three nonoverlapping magnitude bins such that the number of stars with five good measurements is approximately uniform across the bins. The additive error and its uncertainty is then determined for each magnitude bin following the above prescription in the previous paragraph (Figure 15).

Because they describe the mean additional statistical scatter required between epochs, the additive errors $\overline{\delta_{x}}, \overline{\delta_{y}}$ are applied to the position lists at the stage of frame mapping. The distribution of $\chi^{2}$ values from the velocity fits after re-mapping and refitting including additive error was evaluated for three cases: (1) no additive error, (2) a flat additive error (as determined from the $10<K^{\prime}<16$ sample), and (3) additive error allowed to vary with magnitude. We find that a flat distribution of additive error with magnitude produces a velocity $\chi^{2}$ distribution significantly more discrepant from statistical expectation than a magnitudedependent additive error (Figures 16 and 17). We therefore adopt the magnitude-dependent additive error estimate.

\section{B.2. Confusion Bias}

When a sufficiently bright star passes within $\sim 1$ FWHM of the PSF of a star of interest, the shape of its PSF can be
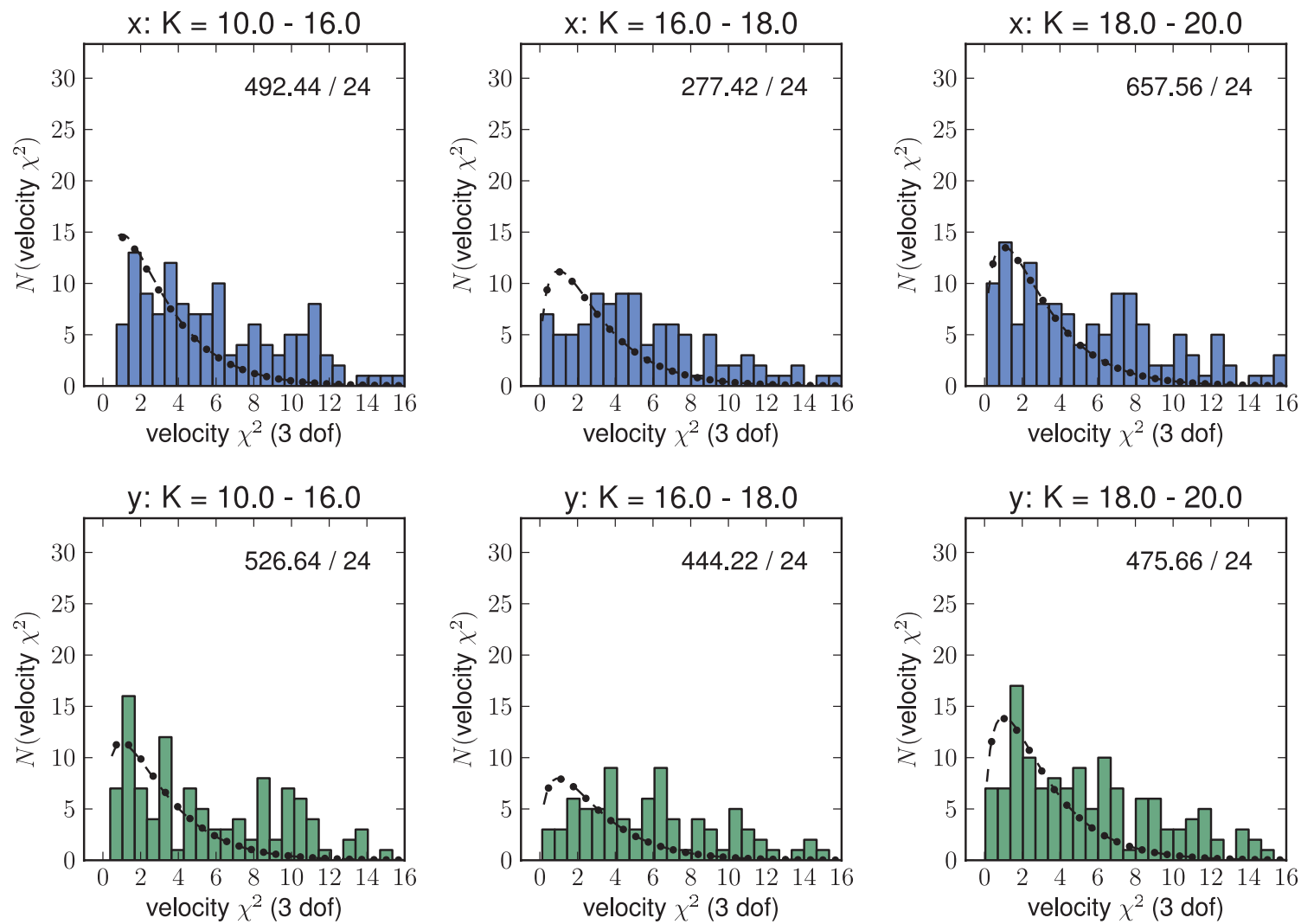

Figure 16. Assessment of the distribution of $\chi^{2}$ from the velocity fits when motions are fit using only centroiding and alignment errors. Columns break the sample of stars into bright, medium, and faint magnitude bins. Histograms show the distribution of velocity fit $\chi^{2}$ values in $X$ (blue; top row) and $Y$ (green; bottom row). The numbers inset give the values of the $\chi^{2}$ test statistic per degree of freedom for the comparison of the observed histogram to the predicted distribution (dashed curve). (A color version of this figure is available in the online journal.) 

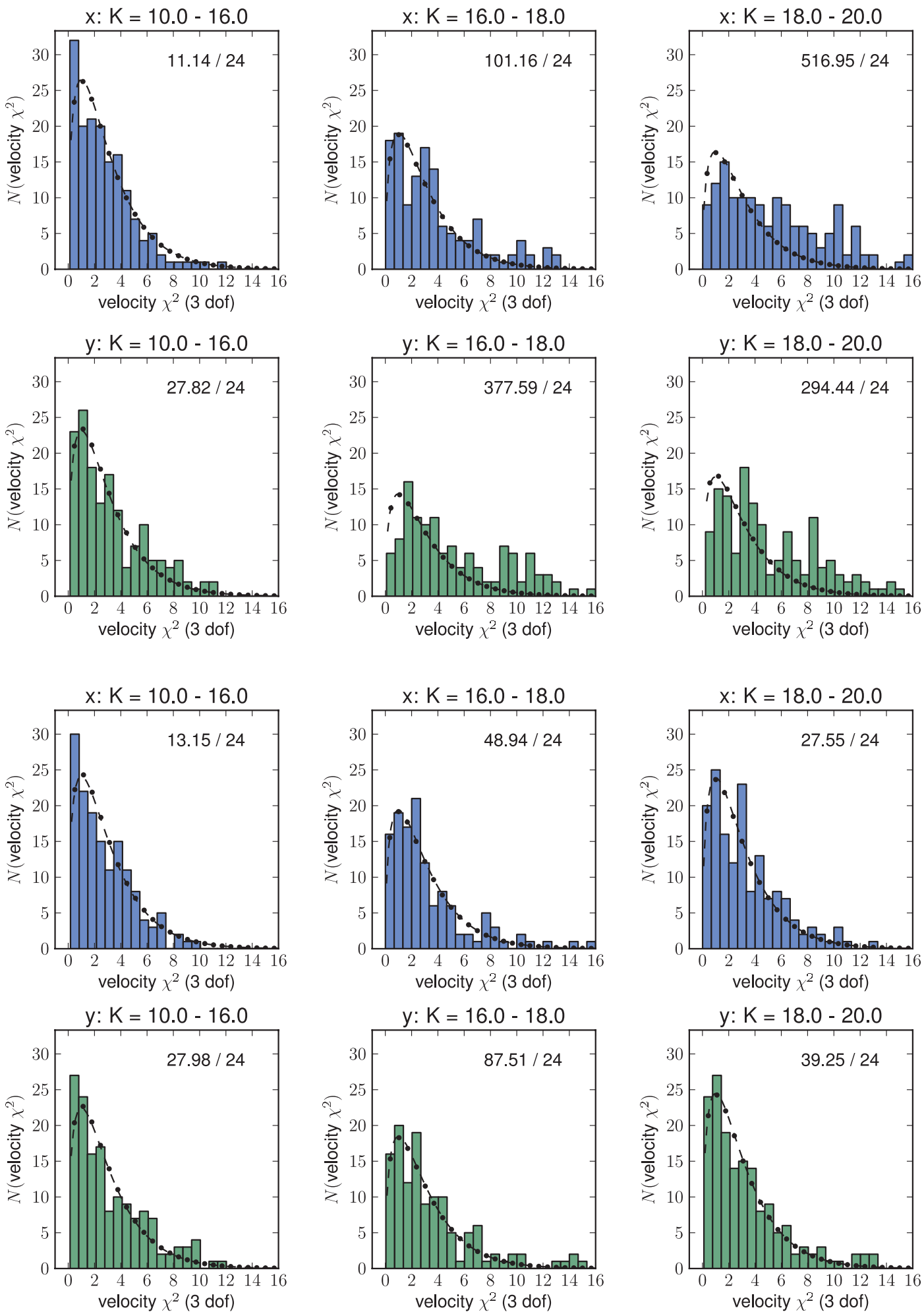

Figure 17. Same as Figure 16, for constant additive error (top) and additive error computed from a fit to separate determinations for each magnitude range (Figure 15).

(A color version of this figure is available in the online journal.)

sufficiently altered that its position measurement is biased, but not so altered that the measurement is rejected. In some cases this bias can be much larger than the positional measurement error ( $\gtrsim 2$ mas for $\Delta K^{\prime}<2$; Ghez et al. 2008). The distribution of this confusion bias across the sample of stars depends on the spatial crowding and magnitude distribution of stars in the field of view. To estimate its order of magnitude for the Arches central field, we use the simulations of Fritz et al. (2010), which model the distribution of astrometric bias as a function of magnitude, for a $K^{\prime}$ distribution appropriate for the nuclear star cluster near the GC. The rms of the confusion bias (denoted here as $\sigma_{\text {bias }}$ ) follows a power law whose normalization depends on the stellar 

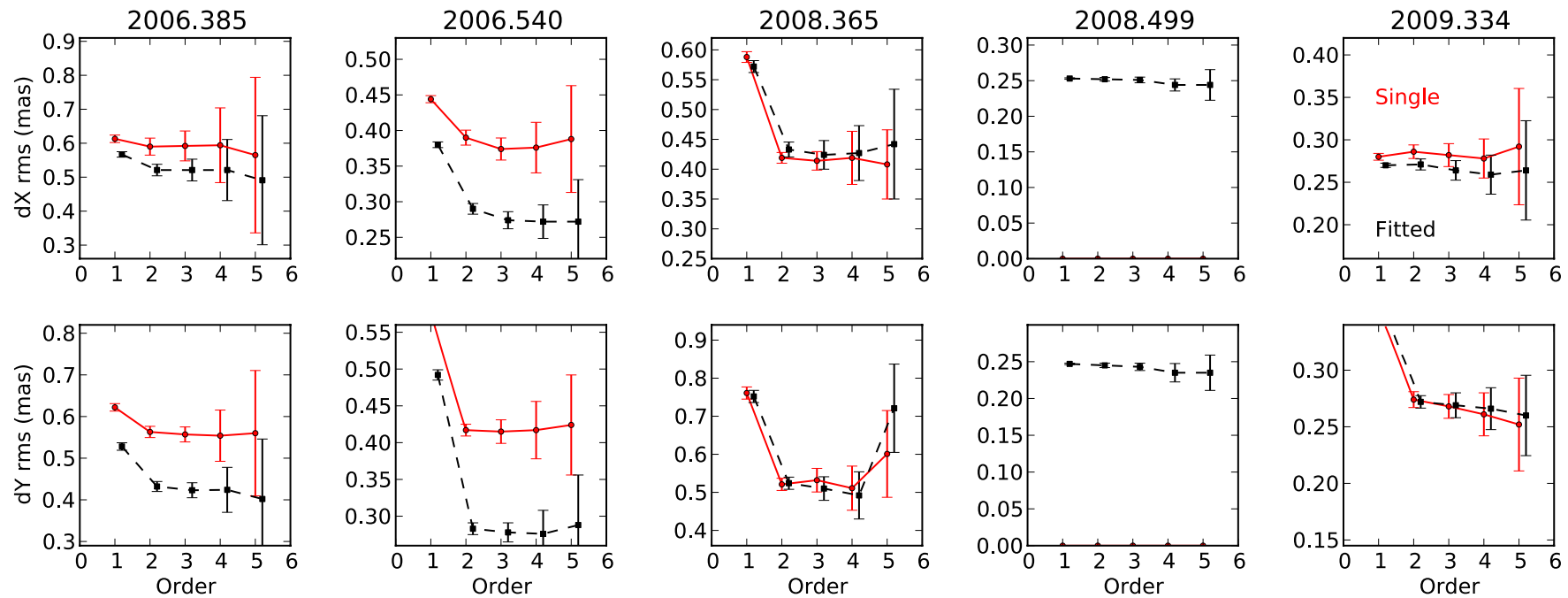

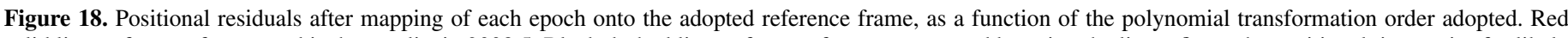

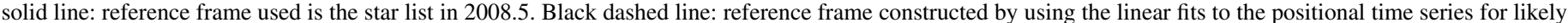

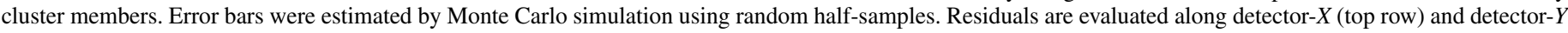
(bottom row).

(A color version of this figure is available in the online journal.)

density within the field. Of their three regions of interest, the stellar density within the Arches field matches most closely that of their 3".5 sample. This then predicts positional bias $\sigma_{\text {bias }}$ of order $20 \%$ of the additive errors $\delta$ for $K^{\prime}<18$ and comparable to $\delta$ at $K^{\prime}>18$ (Table 4 ).

Relative motion across the PSF of the two components of a confused pair would imprint a spurious motion due to the resulting time variation of the confusion bias. Inter-epoch variation in the PSF would thus cause varying positional bias between epochs even in the case of components that are perfectly stationary with respect to each other. Under the expectation that PSF variation between epochs is random, this error is subsumed within the additive random error (Section 3.3.3).

Linear trends in the relative separation of the star and its unrecognized confusing counterpart are in principle more problematic, as the spurious motion thus induced would be impossible to separate from the desired intrinsic motion. Indeed, for some of the rapidly moving S-stars near the GC, apparent deviations from the orbital path on a timescale of up to a few years are clearly visible as the star of interest crosses the region of influence of the confusing source entirely during the time base of the observations (Ghez et al. 2008; Gillessen et al. 2009). Measurements confined to the time interval of confusion would therefore detect linear motion in the wrong direction entirely. However, for the Arches stars of interest here, relative motions of members of a confused pair are too slow to have a significant impact on the motions we measure. We assume that the bias changes by $1 \times \sigma_{\text {bias }}$ in the time taken for the relative separation of confused components to change by the FWHM of the PSF. With expected velocity dispersion $\sim 0.2 \mathrm{mas} \mathrm{yr}^{-1}$ (Stolte et al. 2008), confused pairs of cluster objects change their separation by $\lesssim 3 \%$ of the FWHM per year, so that the astrometric bias will essentially be static for confused cluster pairs. Cluster objects confused with field stars may be subject to relative motions $\sim 5 \mathrm{mas} \mathrm{yr}^{-1}$; in this case the proper motion bias may approach $\sim 0.1 \sigma_{\text {bias }} \mathrm{yr}^{-1}$. We conclude that, for our measurements of motions in the Arches central field, proper motion bias due to confusion trends is a very small effect compared to other sources of error (Table 4) and can safely be ignored in our analysis.

\section{APPENDIX C}

\section{TRANSFORMATION ORDER DURING FRAME MAPPING}

At the level of a few percent of a pixel (comparable to the velocity dispersion we wish to measure), variations in distortion may be present between epochs. These variations might consist of both a spatially random and a spatially correlated part, and might consist of temporally random and/or correlated parts. To quantify spatially correlated time variation, mappings between reference frames were re-fit separately across each $\left(t-t_{0}\right)$ pair using polynomials of the order of $0 \leqslant M \leqslant 5$ using the same set of likely cluster members at each order (Appendix A; step 6). The rms of cluster members in $X$ and $Y$ as transformed to $t_{0}$ was evaluated for each order for each epoch (Figure 18), with errors on the rms evaluated from Monte Carlo resampling and re-fitting in a similar manner to the estimation of alignment errors (Section 3.3.2). Visual inspection suggests that for each epoch, a significant improvement is gained by using a second-order polynomial; order three is sometimes indicated along $Y$, and fourth or higher orders rarely bring about significant improvement.

The formal significance of the improvement of the fit when stepping up from order $M-1$ to $M$ was estimated by evaluating the ratio $\left(\chi_{v}^{2}(M-1)-\chi_{v}^{2}(M)\right) / \chi_{v}^{2}(M)$ for the order of $1<$ $M<5$; assuming that the residuals after mapping are indeed $\chi^{2}$ distributed, this ratio should follow the $F$-distribution for the corresponding pairs of degrees of freedom for $M-1$ and $M$ (e.g., Chapter 11 of Bevington \& Robinson 2003). This produces a formal false-alarm probability that a difference in badness of fit of $\chi_{v}^{2}(M-1)-\chi_{v}^{2}(M)$ or greater could arise from random chance alone. This suggests that order $M>3$ is not warranted for fits to either coordinate (Figure 19, left). The apparent improvement in fit significance at the order of $M=5$ is probably an artifact of overfitting to the $\sim 235$ reference objects ( $M=5$ corresponds to only $\sim 10$ points per term in the polynomial).

A control test was conducted where stars at the observed positions were moved randomly under the expected velocity distribution of the cluster or (for $15 \%$ of objects) the field, perturbed by measurement error, and subjected to a second-order polynomial of similar amplitude to the 

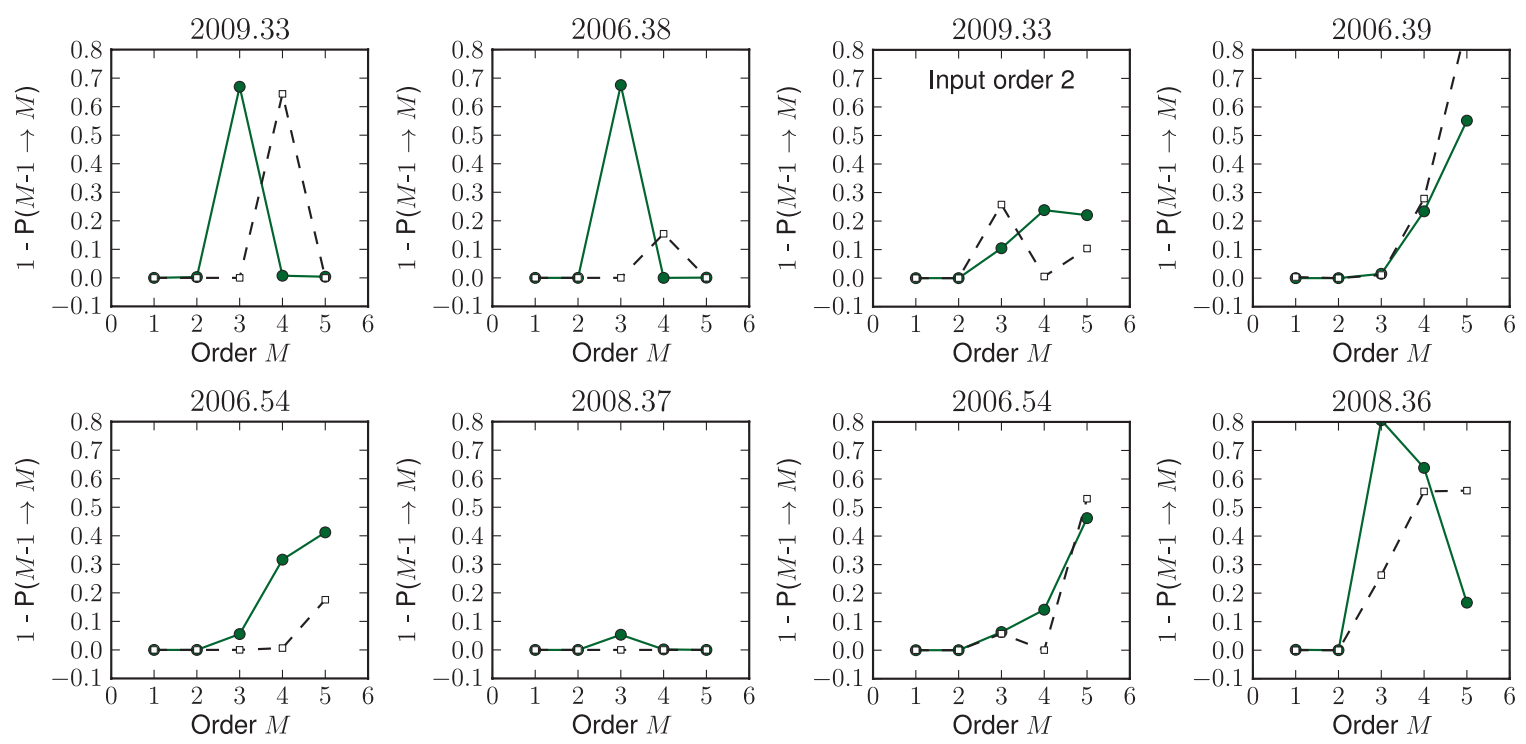

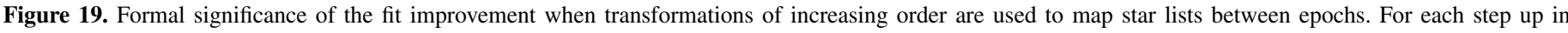

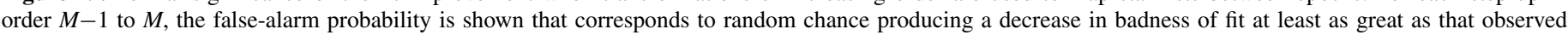

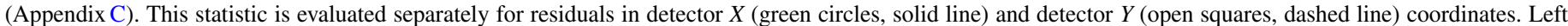

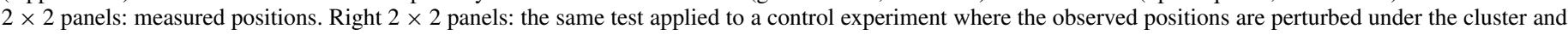

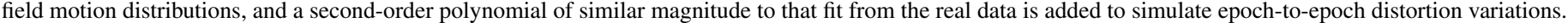
A polynomial of the order of two produces a formally significant improvement in the fitting.

(A color version of this figure is available in the online journal.)

parameters fitted to the real stellar positions. This indicated that the formal fit statistic is indeed sensitive to the polynomial order, provided the number of reference stars is sufficient. A fifth-order polynomial (21 terms, or $\sim 11$ stars per term) is often spuriously indicated (Figure 19, right). We therefore adopt a second-order polynomial for the frame mapping when extracting motions. In principle, relative distortions between epochs might require a more complicated description, but this cannot be determined from the sample at hand.

\section{APPENDIX D}

\section{MISCLASSIFICATION BIAS IN KINEMATIC FITTING}

Section 4.1.1 details the steps taken to estimate membership probabilities by simultaneously fitting cluster and field kinematic properties (denoted $\overline{\boldsymbol{\mu}_{k}}, \boldsymbol{\Sigma}_{k}$ for each component) and membership fraction $\left(\pi_{k}\right)$. Because of the interrelation between $\pi_{k}$ and $\left(\overline{\boldsymbol{\mu}}_{k}, \pi_{k}\right)$, any biases in fitting the kinematic components translate into biases in the membership probabilities, and vice versa. To mitigate the effect of magnitude-dependent error on $\pi_{k}$, as well as allow for any intrinsic changes in $\mathbf{Z}_{k}$ with magnitude, the maximum likelihood fitting was carried out in a magnitude-dependent way as described in Section 4.1.1.

To investigate the size of any misclassification biases, synthetic data sets were simulated using the same parameters $\left(\overline{\boldsymbol{\mu}}_{k}, \boldsymbol{\Sigma}_{k}, \pi_{k}\right)$ at all magnitudes and perturbed by proper motion errors sampled from the magnitude-error curves observed (Figure 2). The fitting process in Section 4.1.1 was performed for a large number of trials and the recovered parameters observed as a function of magnitude. For this set of tests, errors on the recovered parameters are the standard deviation of the parameters recovered over the trials. For each trial data set, the fitting process was carried out using tracer stars selected according to two schemes: (1) using all stars regardless of brightness and (2) the magnitude-local scheme described in Section 4.1. Figure 20 shows the result. Both techniques show some degree of bias at fainter magnitudes (higher errors), though as the sample is usually dominated by the faintest stars in each sample, the biases are comparable.

\section{APPENDIX E}

\section{DYNAMICAL MASS ESTIMATE}

Many proper motion data sets (including that reported here) cover only the inner region of the cluster, over which the velocity anisotropy varies too slowly with radius to be well constrained by the proper motions. In this case the popular moment-based estimator of Leonard \& Merritt (1989, their Equation (19)) should not be used (as pointed out by LM89). A full nonparametric modeling of the data set (see Schödel et al. 2009) is not appropriate without data spanning a wider radius range than we have at present. Instead we use a prescription for the mass density $\rho(r)$ and evaluate the predicted velocity dispersion profile for comparison to our dispersion data.

\section{E.1. Model and Method}

For this first examination we assume the cluster can be adequately parameterized by an isotropic, spherical King (1962) model. In this limit, the model is completely described by three parameters; the core radius $R_{c}$, the tidal radius $R_{t}$, and the total cluster mass $M_{\mathrm{cl}}$. Model parameters were varied over a grid of values, with the velocity dispersion profile projected onto the sky and compared to our data set in each case. The variation of $\chi^{2}$ with parameter values was then used to estimate confidence limits on the model parameters, as discussed in the main text.

The kinematic data set covers the innermost region of the cluster, within $\sim 1-2 \times R_{c}$. For this reason the shape parameters of the King profile are poorly constrained by the kinematic data alone. We therefore evaluate $\chi^{2}$ in two ways and present confidence limits derived from both. First, ranges are estimated comparing model predictions to kinematic data alone. Second, ranges are estimated by comparing predictions to the kinematic 

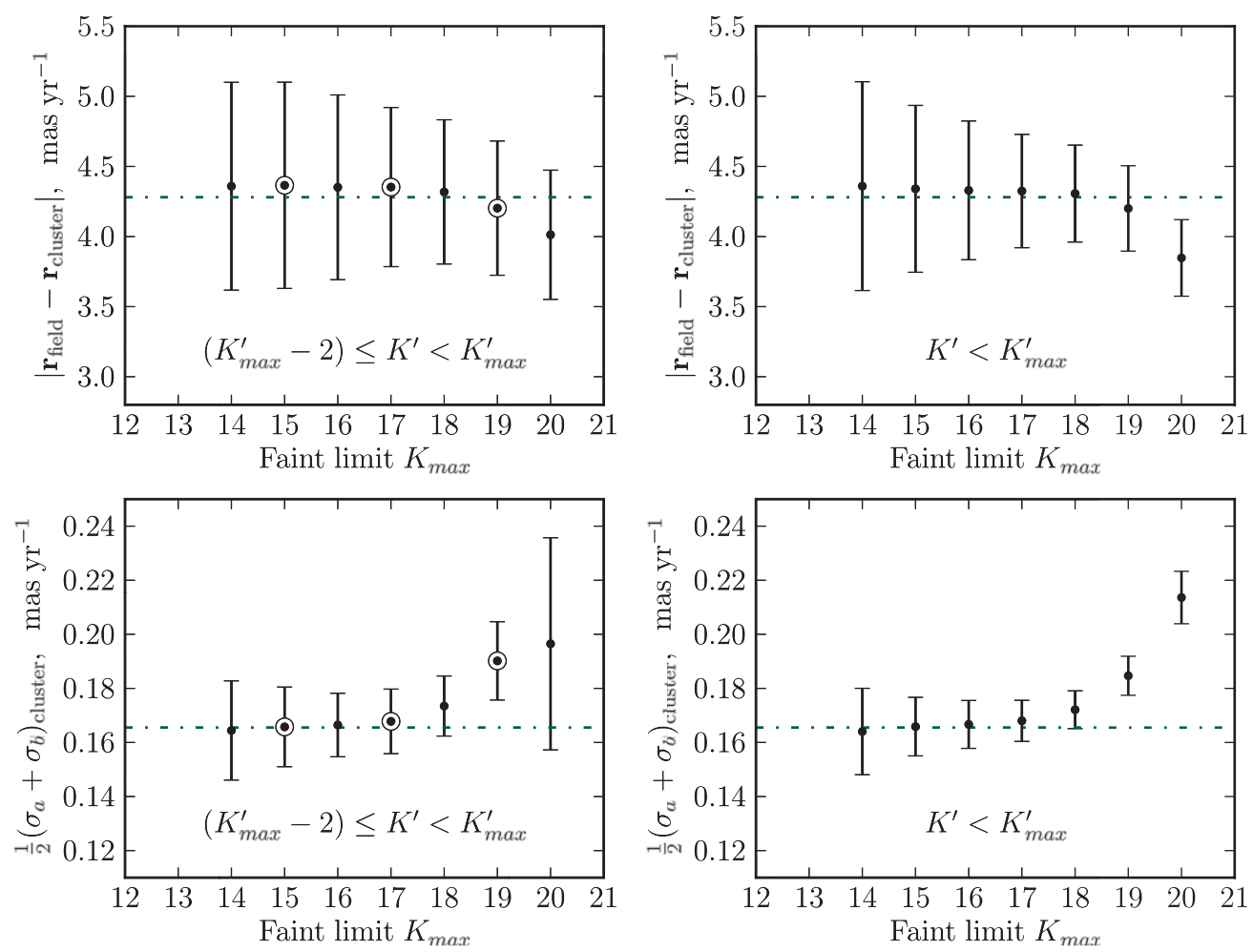

Figure 20. Dependence of fitted kinematic parameters on star brightness. Magnitudes and errors were sampled from the observed magnitude and error distribution (bottom right panel of Figure 2). Two measures are shown: the separation between components in the VPD (top row) and the average of the cluster major and minor axes (bottom row). The same underlying kinematic parameters were used for all simulations in this figure (green broken lines). The left column shows fits evaluated over two-magnitude-wide magnitude bins (with non-overlapping bins indicated using the symbols); the right column shows fits evaluated over all stars brighter than $K^{\prime}$ in each bin. If all stars are fit together to estimate kinematic parameters for the cluster as a whole (corresponding to the faintest bin in the right column) then the fitted parameters are biased.

(A color version of this figure is available in the online journal.)

data set and also to the surface density by number $\Sigma_{N}(R)$ for massive stars. Espinoza et al. (2009) report $\Sigma_{N}(R)$ profiles for three mass ranges: $(10 \leqslant M<30) M_{\odot},\left(30 \leqslant M \leqslant 120 M_{\odot}\right)$ and the union of the two, $\left(10 \leqslant M \leqslant 120 M_{\odot}\right)$. We examined both the full mass range and the $\left(10 \leqslant M \leqslant 30 M_{\odot}\right)$ mass range when comparing $\Sigma_{N}(R)$ to data, to gain insight into the dependence of the derived mass on the shape parameters of the cluster. Two grids were used to explore parameter space, one coarse:

1. $\left(0.05 \leqslant R_{c} \leqslant 0.8\right)$ pc in 40 steps;

2. $\left(1.0 \leqslant R_{t} \leqslant 50.0\right) \mathrm{pc}$ in 40 steps;

3. $\left(0.5 \leqslant M_{\mathrm{cl}} \leqslant 10.0\right) \times 10^{4} M_{\odot}$ in 40 steps

the other somewhat more finely spaced near the apparent $\chi^{2}$ minimum:

4. $\left(0.05 \leqslant R_{c} \leqslant 0.8\right)$ pc in 50 steps;

5. $\left(1.0 \leqslant R_{t} \leqslant 30.0\right)$ pc in 50 steps;

6. $\left(0.5 \leqslant M_{\mathrm{cl}} \leqslant 6.0\right) \times 10^{4} M_{\odot}$ in 50 steps.

Each $R_{c}, R_{t}, M_{\mathrm{cl}}$ combination predicts a pattern of surface density by mass $\Sigma_{\text {mass }}(R)$ (units $M_{\odot} \mathrm{pc}^{-2}$ ), which is optimally scaled to the surface density by number $\Sigma_{N}(R)$ (units stars pc $\left.{ }^{-2}\right) .^{18}$ This scale factor $\Sigma_{N, 0} / \rho_{0}$ relates to the mass function of the cluster, and so we include it in the reported quantities derived from the model parameters.

18 As this scale factor is optimized to fit the data for each trial set of the other three parameters, the appropriate $\Delta \chi^{2}$ regions for significance ranges are unchanged from the kinematic-only comparison; three parameters are allowed to vary.
With a few exceptions, observational mass estimates report the total mass in stars in a cylinder of radius $R=0.35-0.4 \mathrm{pc}$ on-sky (Section 5.4). Hereafter we refer to this quantity as the "projected mass" $M(<R)$ to distinguish it from the total mass enclosed within a sphere of radius $r$, i.e., $M(<r)$. We report confidence limits on $M(R<0.4 \mathrm{pc})$ to provide a direct comparison with the observational literature. We also report limits on $M(r<1.0 \mathrm{pc})$, as well as the total cluster mass $M_{\mathrm{cl}}$ (when all three model parameters are allowed to vary). For interest we also report the central volume density $\rho_{0}$ in the table of confidence regions.

The form for $M(R<0.4 \mathrm{pc})$ can be analytically derived from the form for $\Sigma_{\text {mass }}(R)$. It is reported in King (1962); for convenience we repeat the form here:

$$
\begin{aligned}
M(<R) & =\pi R_{c}^{2} K\left[\ln (1+x)-4 \frac{\sqrt{1+x}-1}{\sqrt{1+x_{t}}}+\frac{x}{1+x_{t}}\right], \\
x & \equiv\left(R / R_{c}\right)^{2} \\
x_{t} & \equiv\left(R_{t} / R_{c}\right)^{2}
\end{aligned}
$$

while $M(r<1.0 \mathrm{pc})$ is estimated from

$$
M(<r)=4 \pi \int_{0}^{r=1 \mathrm{pc}} r^{2} \rho(r) d r,
$$

and $\rho_{0}$ is evaluated by section $r=0$ in Equation (5).

\section{E.2. Results}

Figures 21 and 22 show the behavior of the $95 \%$ significance region in $R_{c}, R_{t}, M_{\mathrm{cl}}$ and $R_{c}, R_{t}, M(R<0.4 \mathrm{pc})$ space, 


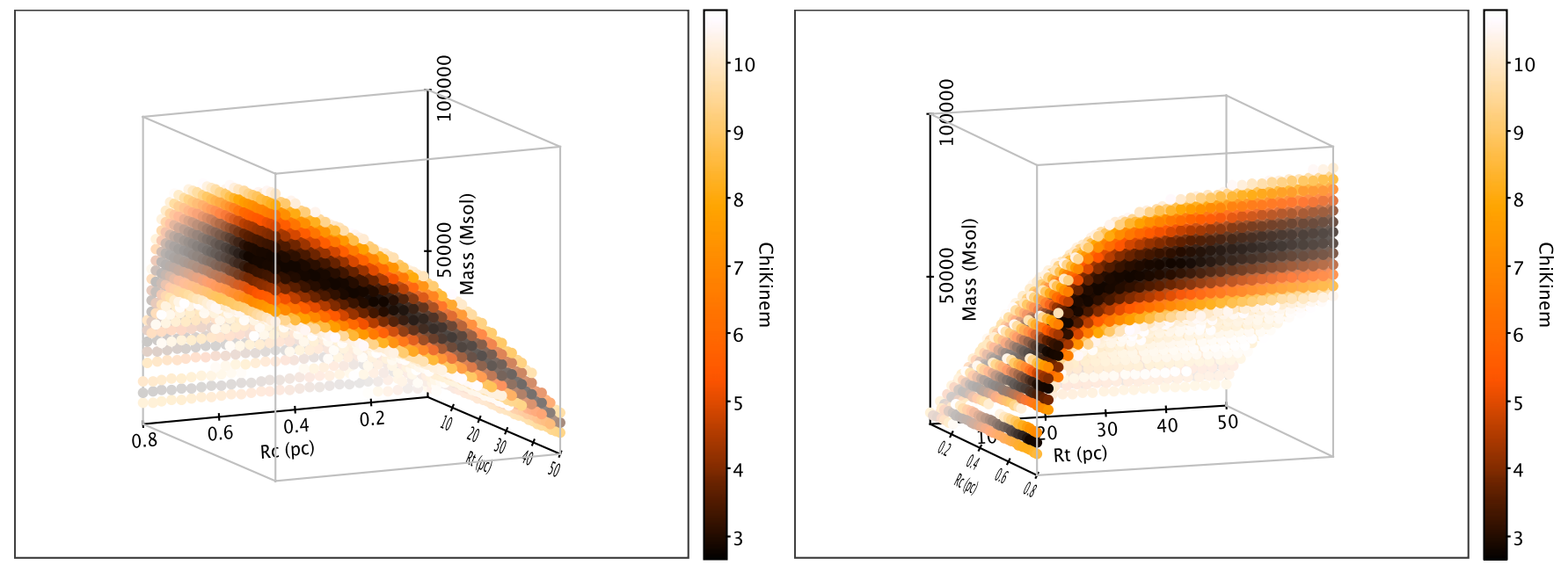

Figure 21. Views of the $\Delta \chi_{\text {kinem }}^{2}<7.82$ region when only the kinematic data set is used to assess the mass model. Axes are: $R_{c}, R_{t}, M_{\mathrm{cl}}$, with total cluster mass $M_{\mathrm{cl}}$ vertical in each case. Limits shown are: $0.05 \leqslant R_{c} \leqslant 0.8 \mathrm{pc}, 1.0 \leqslant R_{t} \leqslant 50 \mathrm{pc}, 0.5 \leqslant M_{\mathrm{cl}} \leqslant 10.0 \times 10^{4} M_{\odot}$.

(A color version of this figure is available in the online journal.)
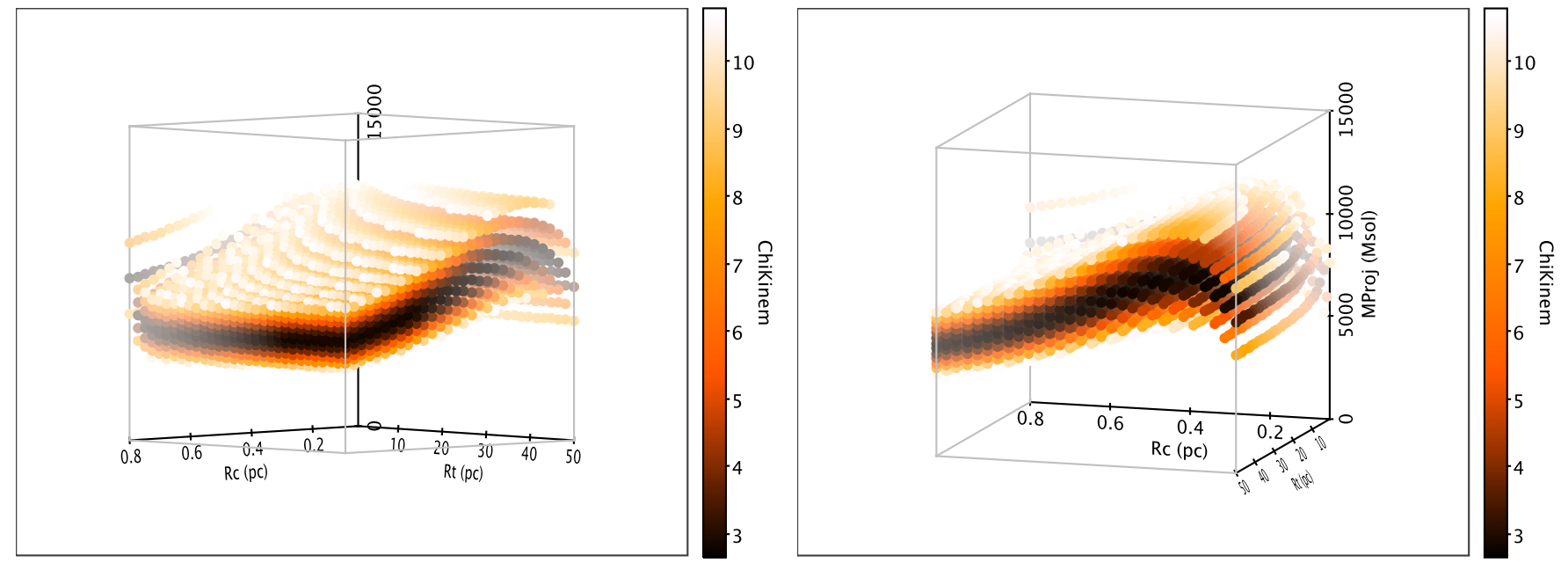

Figure 22. Same as Figure 21 , but with $M(R<0.4 \mathrm{pc})$ along the vertical axis. Limits shown are: $0.05 \leqslant R_{c} \leqslant 0.8 \mathrm{pc}, 1.0 \leqslant R_{t} \leqslant 50 \mathrm{pc}, 0.0 \leqslant M(R<0.4 \mathrm{pc}) \leqslant$ $1.5 \times 10^{4} M_{\odot}$.

(A color version of this figure is available in the online journal.)

respectively, for the coarse grid of parameter values and without using any constraints on the surface density $\Sigma_{N}(R)$. Our maximum tidal radius $R_{t}=50 \mathrm{pc}$ already would suggest a very extended cluster, and it is likely that increasing $R_{t}$ still further would decrease $M(R<0.4 \mathrm{pc})$. Although the total mass and shape parameters of the King (1962) model are poorly constrained by kinematic data alone, the requirement that the cluster arrange itself in order to yield the low velocity dispersion that we measure, imposes an upper limit to $M(R<0.4 \mathrm{pc})$ of about $1.30 \times 10^{4} M_{\odot}$. With this data set only, the total cluster mass is essentially unconstrained (Table 11). Figure 23 shows radial profiles drawn from within the $\Delta \chi_{\text {kinem }}^{2}=7.82$ surface in parameter space.

The interdependence of the model parameters when representing the observed dispersions can be intuitively understood in the following way: under a King model, for constant tidal radius $R_{t}$, a more centrally concentrated cluster (i.e., smaller $R_{c}$ ) produces a larger projected velocity dispersion for the same cluster mass $M_{\mathrm{cl}}$. Thus, to match the observed dispersion plateau we measure, without $\Sigma_{N}(R)$ information the total cluster mass $M_{\mathrm{cl}}$ should decrease as $R_{c}$ decreases at constant $\chi_{\text {kinem }}^{2}$, as is ob- served (Figure 21). However, when evaluating $M(R<0.4 \mathrm{pc})$, we see that as $R_{c}$ is increased, a smaller fraction of the total cluster mass is observed within a cylinder of radius $R=0.4 \mathrm{pc}$ on the sky, while $M_{\mathrm{cl}}$ increases as $R_{c}$ increases. The tradeoff between these two trends leads to the shape of the $\chi_{\text {kinem }}^{2}$ surface for $M(R<0.4 \mathrm{pc})$.

Inclusion of the $\Sigma_{N}(R)$ data produces tighter constraints on the derived parameters because the cluster shape is now more tightly constrained (Tables 10 and 12 and Figures 24 and 25; Figure 26 shows radial profiles in this case). In this latter case, the upper limit on $M(R<0.4 \mathrm{pc})$ is still about $1.30 \times 10^{4} M_{\odot}$, but now the lower limit is increased and the total cluster mass is itself constrained, to $M(r<1.0 \mathrm{pc})=1.5_{-0.60}^{+0.74} \times 10^{4} M_{\odot}$ at $99.7 \%$ confidence.

\section{E.3. Beyond the Isotropic King Model}

The isotropic King (1962) profile assumes that the cluster has achieved dynamical relaxation through multiple collisions between its constituent stars. While the Arches cluster is young compared to a typical crossing time of most of its stars, the 
Isotropic King profiles from 95\% confidence region - Kinematics only
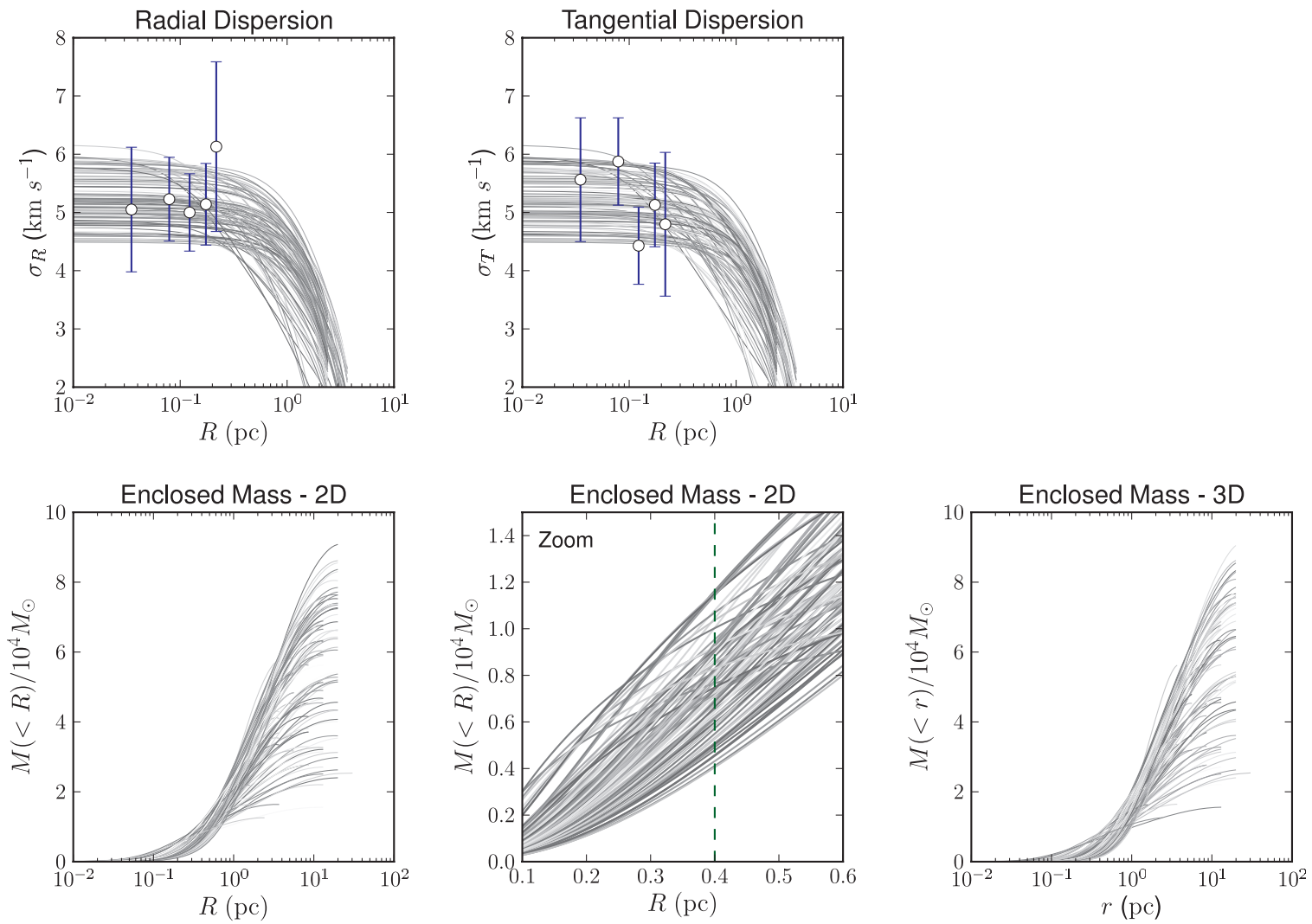

Figure 23. Radial profiles corresponding to parameter sets within the $\Delta \chi_{\text {kinem }}^{2}<7.82$ surface. Top left and top middle panels show radial and tangential velocity dispersions from proper motions (points) over the projected profiles corresponding to model parameters (lines). Bottom left and bottom middle panels show the total mass within cylindrical radius $R$ on the sky, with $R=0.4 \mathrm{pc}$ indicated by the vertical dashed line. Bottom right panel shows the mass enclosed within a sphere of radius $r$ pc from the cluster center. See also Table 11.

(A color version of this figure is available in the online journal.)
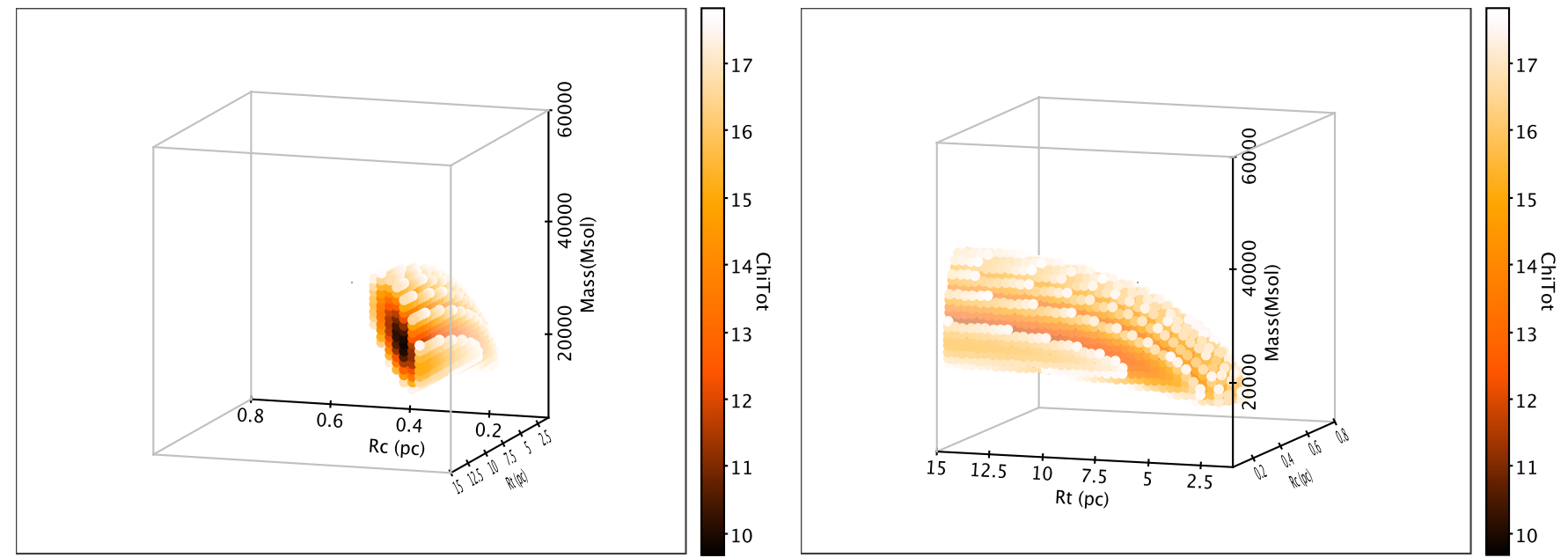

Figure 24. Views of the $\Delta \chi_{\text {full }}^{2}<7.82$ region when both kinematic and surface density data (using the full mass range of Espinoza et al. 2009) are included in the assessment. Axes are: $R_{c}, R_{t}, M_{\mathrm{cl}}$, with total cluster mass $M_{\mathrm{cl}}$ vertical in each case. Limits shown are: $0.05 \leqslant R_{c} \leqslant 0.8 \mathrm{pc}, 1.0 \leqslant R_{t} \leqslant 15 \mathrm{pc}$, $0.5 \leqslant M_{\mathrm{cl}} \leqslant 6.0 \times 10^{4} M_{\odot}$.

(A color version of this figure is available in the online journal.)

cluster is likely to be significantly older dynamically than this consideration would suggest, since it has likely already undergone gravitational collapse (Allison et al. 2009). Thus, we selected a King (1962) model as a reasonable first-order approximation.
Full constraints await realistic $N$-body simulations using our dispersion measurements as a constraint. In the meantime, we attempted to follow the practical algorithm of LM89 for a (possibly strongly) anisotropic cluster. In their approach, a velocity dispersion profile $\sigma_{\text {iso }}^{2}(r)$ is computed under the 
Table 10

Significance Regions for Isotropic King Modeling of the Arches Cluster

\begin{tabular}{|c|c|c|c|}
\hline $\begin{array}{l}\Delta \chi_{\text {full }}^{2} \\
\text { Confidence }\end{array}$ & $\begin{array}{l}3.50 \\
68 \% \\
" 1 \sigma "\end{array}$ & $\begin{array}{l}7.82 \\
95 \% \\
\text { " } 2 \sigma "\end{array}$ & $\begin{array}{c}13.93 \\
99.7 \% \\
\text { " } 3 \sigma "\end{array}$ \\
\hline $\begin{array}{l}M(R<0.40 \mathrm{pc}) \\
\left(10^{4} M_{\odot}\right)\end{array}$ & $0.69-1.10$ & $0.62-1.20$ & $0.55-1.30$ \\
\hline $\begin{array}{l}M(r<1.0 \mathrm{pc}) \\
\left(10^{4} M_{\odot}\right) \\
\end{array}$ & $1.16-1.88$ & $1.04-2.06$ & $0.91-2.24$ \\
\hline $\begin{array}{l}\rho_{0} \\
\left(10^{5} M_{\odot} \mathrm{pc}^{-3}\right)\end{array}$ & $0.45-1.66$ & $0.30-2.34$ & $0.20-3.19$ \\
\hline $\begin{array}{l}R_{c} \\
(\mathrm{pc})\end{array}$ & $0.18-0.31$ & $0.15-0.44$ & $0.13-0.80$ \\
\hline $\begin{array}{l}R_{t} \\
(\mathrm{pc})\end{array}$ & $2.00-30.00$ & $2.00-30.00$ & $1.00-30.00$ \\
\hline $\begin{array}{l}M_{\text {cluster }} \\
\left(10^{4} M_{\odot}\right)\end{array}$ & $1.64-4.29$ & $1.45-4.86$ & $1.07-5.62$ \\
\hline $\begin{array}{l}1000 \times \Sigma_{N, 0} / \rho_{0} \\
\left(\text { stars }^{-2} / M_{\odot} \mathrm{pc}^{-3}\right)\end{array}$ & $0.00-0.07$ & $0.00-0.18$ & $0.00-15.62$ \\
\hline
\end{tabular}

Notes. Ranges of each parameter corresponding to the stated significance level are given, when $R_{c}, R_{t}, M_{\text {cluster }}$ are all allowed to vary. The quantity $\chi_{\text {full }}^{2}$ denotes the badness of fit when comparing model predictions to both the Arches kinematic data set and the surface density data set of Espinoza et al. (2009), over the mass range $(10 \leqslant M \leqslant 30) M_{\odot}$.

assumption that the cluster is isotropic and follows some density model $n(r)$ that is fit to the subset of stars directly observed. The isotropic dispersion profile $\sigma_{\text {iso }}^{2}$ is then modified following some prescription for anisotropic velocity dispersions to produce radial and tangential dispersions $\sigma_{r}^{2}, \sigma_{t}^{2}$. The enclosed mass $M(<r)$ and underlying mass density $\rho(r)$ are then derived from the Jeans equation for dispersions $\sigma_{r}^{2}, \sigma_{t}^{2}$ of tracer stars whose number density distribution follows $n(r)$. Finally, the quantities of interest (particularly $M(R<0.4 \mathrm{pc})$ ) can be calculated from the model, for example by projecting $\rho(r)$ onto the sky and integrating over projected radius $R$.

LM89 use an isotropic King (1962) profile to generate $n(r)$ and $\sigma_{\text {iso }}^{2}$. To modify the dispersion for an anisotropic cluster, they raise $\sigma_{\text {iso }}$ to some power $N_{r}, N_{t}$ for radial and tangential dispersions, respectively. This allows for an extreme range of anisotropies (provided only $N_{r}, N_{t}$ combinations that yield positive $\rho(r)$ everywhere are used). When we attempted this procedure, we found that the first term in the Jeans equation diverges strongly for significant regions of parameter space, although we reproduce the shapes of the velocity dispersion profiles of LM89 exactly (Figure 27).

We also attempted the approach of Leonard et al. (1992), in which the general method of LM89 is modified by using a Plummer profile to describe $n(r)$ and $\sigma_{\text {iso }}^{2}(r)$. In this case we were able to reproduce the Leonard et al. (1992) dispersion curves exactly for anisotropic models without the divergence problems we encountered estimating $M(<r)$ following LM89. Under a Plummer model the Arches cluster is more centrally concentrated than the relaxed King (1962) profile. Figure 28 shows radial profiles drawn from the $\Delta \chi_{\text {full }}^{2}=7.82$ regions that result. Because the radial profile of the Plummer model provides a poorer fit to $\Sigma_{N}(R)$ than the King (1962) profile, a wide range of radial profiles are consistent with the data. In this case the best-fitting values for $M(R<0.4 \mathrm{pc})$ are slightly broader than for the King (1962) profile, but still below $1.5 \times 10^{4} M_{\odot}$ for all combinations within the formal $2 \sigma$ confidence region.
Table 11

Significance Regions for Isotropic King Modeling of the Arches Cluster

\begin{tabular}{|c|c|c|c|}
\hline $\begin{array}{l}\Delta \chi_{\text {kinem }}^{2} \\
\text { Confidence }\end{array}$ & $\begin{array}{l}3.50 \\
68 \% \\
\text { "1 } \sigma \text { " }\end{array}$ & $\begin{array}{l}7.82 \\
95 \% \\
\text { " } 2 \sigma \text { " }\end{array}$ & $\begin{array}{c}13.93 \\
99.7 \% \\
\text { " } 3 \sigma "\end{array}$ \\
\hline $\begin{array}{l}M(R<0.40 \mathrm{pc}) \\
\left(10^{4} M_{\odot}\right)\end{array}$ & $0.40-1.10$ & $0.36-1.19$ & $0.32-1.30$ \\
\hline $\begin{array}{l}M(r<1.0 \mathrm{pc}) \\
\left(10^{4} M_{\odot}\right)\end{array}$ & $0.74-1.98$ & $0.50-2.14$ & $0.50-2.33$ \\
\hline $\begin{array}{l}\rho_{0} \\
\left(10^{5} M_{\odot} \mathrm{pc}^{-3}\right)\end{array}$ & $0.06-30.92$ & $0.05-30.92$ & $0.05-31.40$ \\
\hline $\begin{array}{l}R_{c} \\
(\mathrm{pc})\end{array}$ & $0.05-0.80$ & $0.05-0.80$ & $0.05-0.80$ \\
\hline $\begin{array}{l}R_{t} \\
(\mathrm{pc})\end{array}$ & $1.00-50.00$ & $1.00-50.00$ & $1.00-50.00$ \\
\hline $\begin{array}{l}M_{\text {cluster }} \\
\left(10^{4} M_{\odot}\right)\end{array}$ & $0.83-8.36$ & $0.50-9.34$ & $0.50-10.00$ \\
\hline
\end{tabular}

Notes. Ranges of each parameter corresponding to the stated significance level are given, when $R_{c}, R_{t}, M_{\text {cluster }}$ are all allowed to vary. The quantity $\chi_{\text {kinem }}^{2}$ denotes the badness of fit when comparing model predictions to the Arches kinematic data set only.

Table 12

Significance Regions for Isotropic King Modeling of the Arches Cluster

\begin{tabular}{|c|c|c|c|}
\hline $\begin{array}{l}\Delta \chi_{\text {full }}^{2} \\
\text { Confidence }\end{array}$ & $\begin{array}{l}3.50 \\
68 \% \\
\text { " } 1 \sigma "\end{array}$ & $\begin{array}{l}7.82 \\
95 \% \\
\text { " } 2 \sigma \text { " }\end{array}$ & $\begin{array}{c}13.93 \\
99.7 \% \\
" 3 \sigma "\end{array}$ \\
\hline $\begin{array}{l}M(R<0.40 \mathrm{pc}) \\
\left(10^{4} M_{\odot}\right)\end{array}$ & $0.70-1.10$ & $0.62-1.20$ & $0.56-1.30$ \\
\hline $\begin{array}{l}M(r<1.0 \mathrm{pc}) \\
\left(10^{4} M_{\odot}\right)\end{array}$ & $1.07-1.77$ & $0.96-1.92$ & $0.85-2.11$ \\
\hline $\begin{array}{l}\rho_{0} \\
\left(10^{5} M_{\odot} \mathrm{pc}^{-3}\right) \\
\end{array}$ & $0.89-2.37$ & $0.62-3.47$ & $0.44-3.91$ \\
\hline $\begin{array}{l}R_{c} \\
(\mathrm{pc})\end{array}$ & $0.15-0.21$ & $0.13-0.26$ & $0.13-0.33$ \\
\hline $\begin{array}{l}R_{t} \\
(\mathrm{pc})\end{array}$ & $3.00-30.00$ & $2.00-30.00$ & $2.00-30.00$ \\
\hline $\begin{array}{l}M_{\text {cluster }} \\
\left(10^{4} M_{\odot}\right) \\
\end{array}$ & $1.64-3.53$ & $1.26-3.91$ & $1.07-4.48$ \\
\hline $\begin{array}{l}1000 \times \Sigma_{N, 0} / \rho_{0} \\
\left(\text { stars } \mathrm{pc}^{-2} / M_{\odot} \mathrm{pc}^{-3}\right)\end{array}$ & $0.00-0.01$ & $0.00-0.06$ & $0.00-0.12$ \\
\hline
\end{tabular}

Notes. Ranges of each parameter corresponding to the stated significance level are given, when $R_{c}, R_{t}, M_{\text {cluster }}$ are all allowed to vary. The quantity $\chi_{\text {full }}^{2}$ denotes the badness of fit when comparing model predictions to both the Arches kinematic data set and the surface density data set of Espinoza et al. (2009), over the mass range $(10 \leqslant M \leqslant 120) M_{\odot}$.

Further work along these lines would use a more realistic prescription for the anisotropy. Leonard et al. (1992) never justify their approach to modify $\sigma_{\text {iso }}^{2}$ for anisotropy; we note here that it allows for a very large range of velocity dispersion anisotropies yet still produces $M(R<0.4 \mathrm{pc}) \lesssim 1.7 \times 10^{4} M_{\odot}$. Since the King (1962) profile provides a better fit the $\Sigma_{N}(R)$ data set than anisotropic Plummer profiles-and is used to model very young clusters regardless of their evolutionary status (e.g., Harfst et al. 2010) —we report mass ranges corresponding to isotropic King profiles in this paper. We expect that more realistic $N$-body simulations will yield constraints not bound to spherical models. 

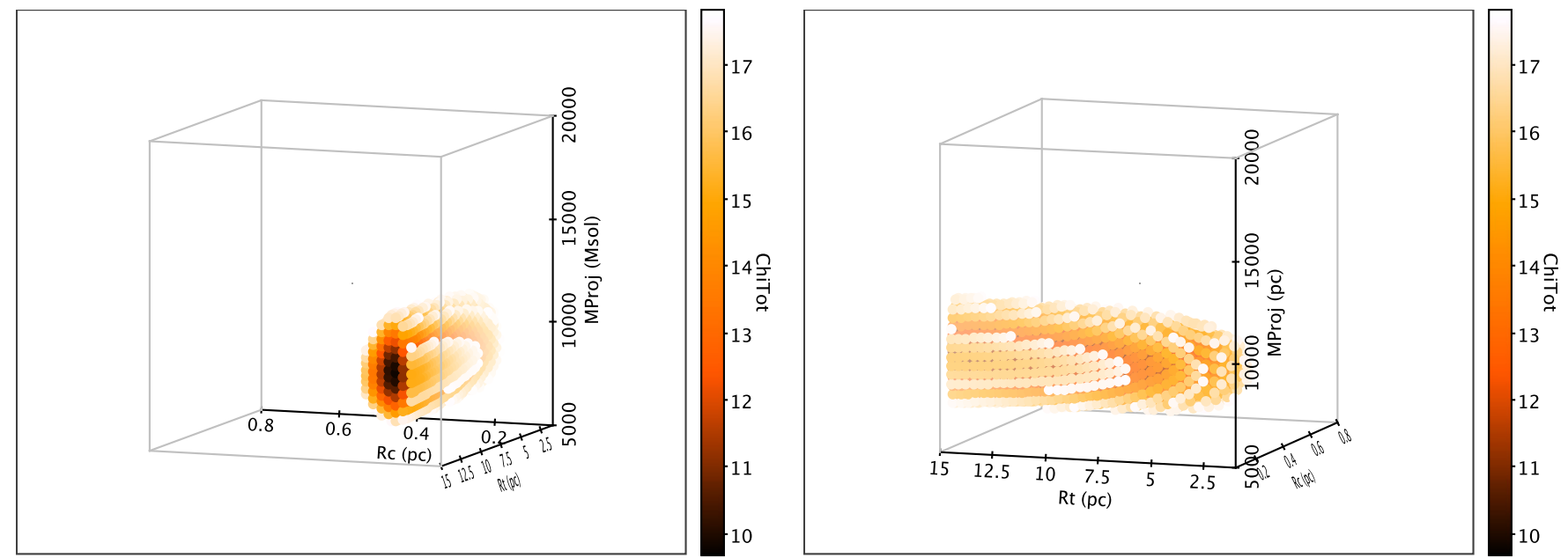

Figure 25. Same as Figure 24, but with $M(R<0.4 \mathrm{pc})$ along the vertical axis. Limits shown are: $0.05 \leqslant R_{c} \leqslant 0.8 \mathrm{pc}, 1.0 \leqslant R_{t} \leqslant 15 \mathrm{pc}, 0.5 \leqslant M(R<0.4 \mathrm{pc}) \leqslant$ $2.0 \times 10^{4} M_{\odot}$.

(A color version of this figure is available in the online journal.)

\section{Isotropic King profiles from 95\% confidence region}
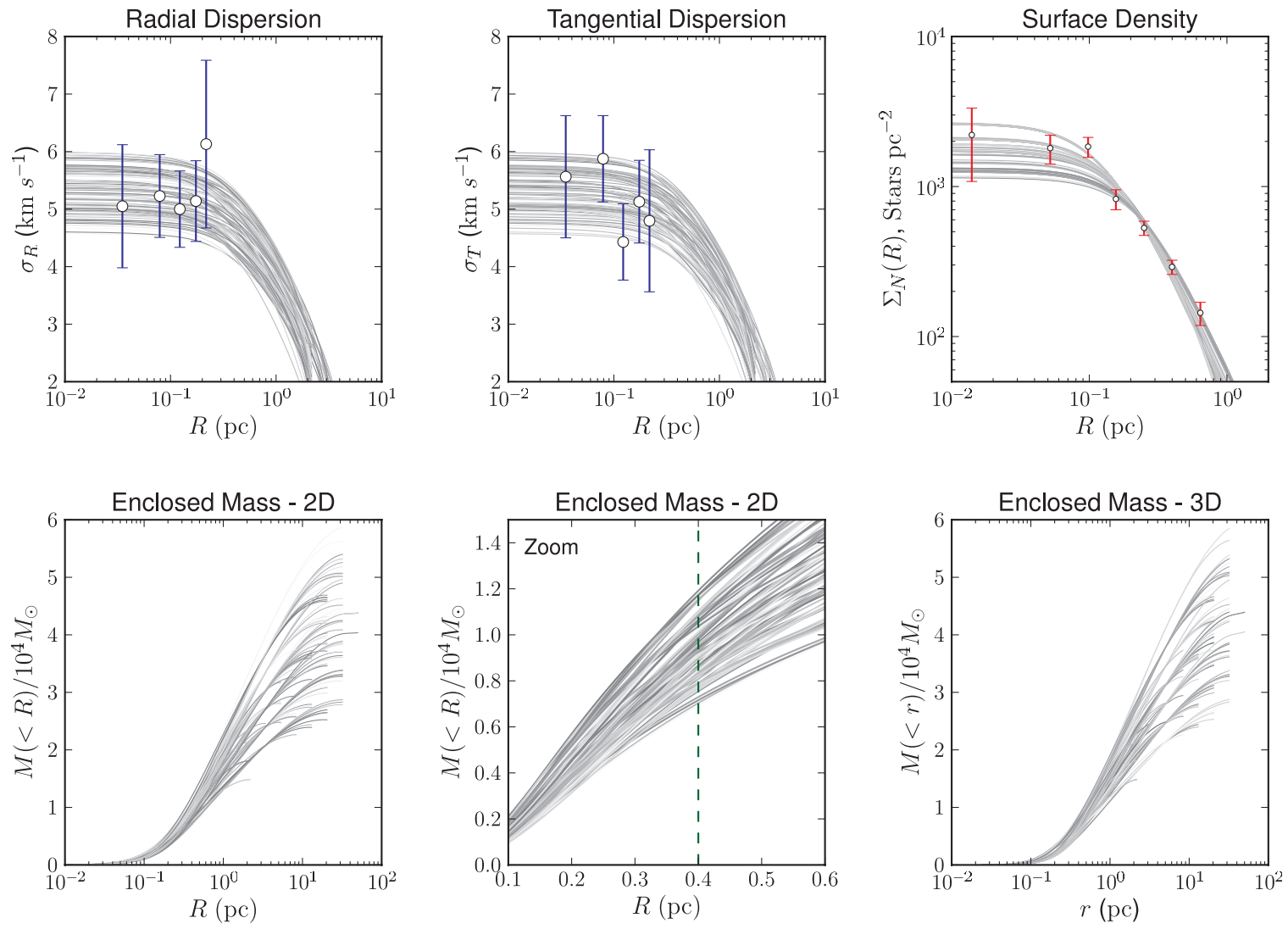

Figure 26. Same as Figure 10 , but this time $\Sigma_{N}(R)$ corresponds to stars in the range $(10 \leqslant M \leqslant 120) M_{\odot}$. See Table 12 .

(A color version of this figure is available in the online journal.)

\section{E.4. Functional Forms in the Leonard et al. (1992) Approach}

Leonard et al. (1992) choose not to provide the functional forms of several of the steps in their analysis. To aid the reader who might be interested to try this approach, we show the relevant relationships here. Leonard et al. (1992) assume that the measured stars are distributed with an isotropic spatial distribution $n(r)$, but the same stars move in a way that reflects the true underlying mass distribution and thus may show anisotropic motion. This motion is parameterized as a modified form of the velocity dispersion that would be obtained if the motions were isotropic. The flow from model to derived quantities is:

1. Start with a prescription for the number density of tracers $n(r)$ for an isotropic distribution, and derive the velocity dispersion profile that would be obtained if motions were isotropic. For the Plummer model of Leonard et al. (1992) 

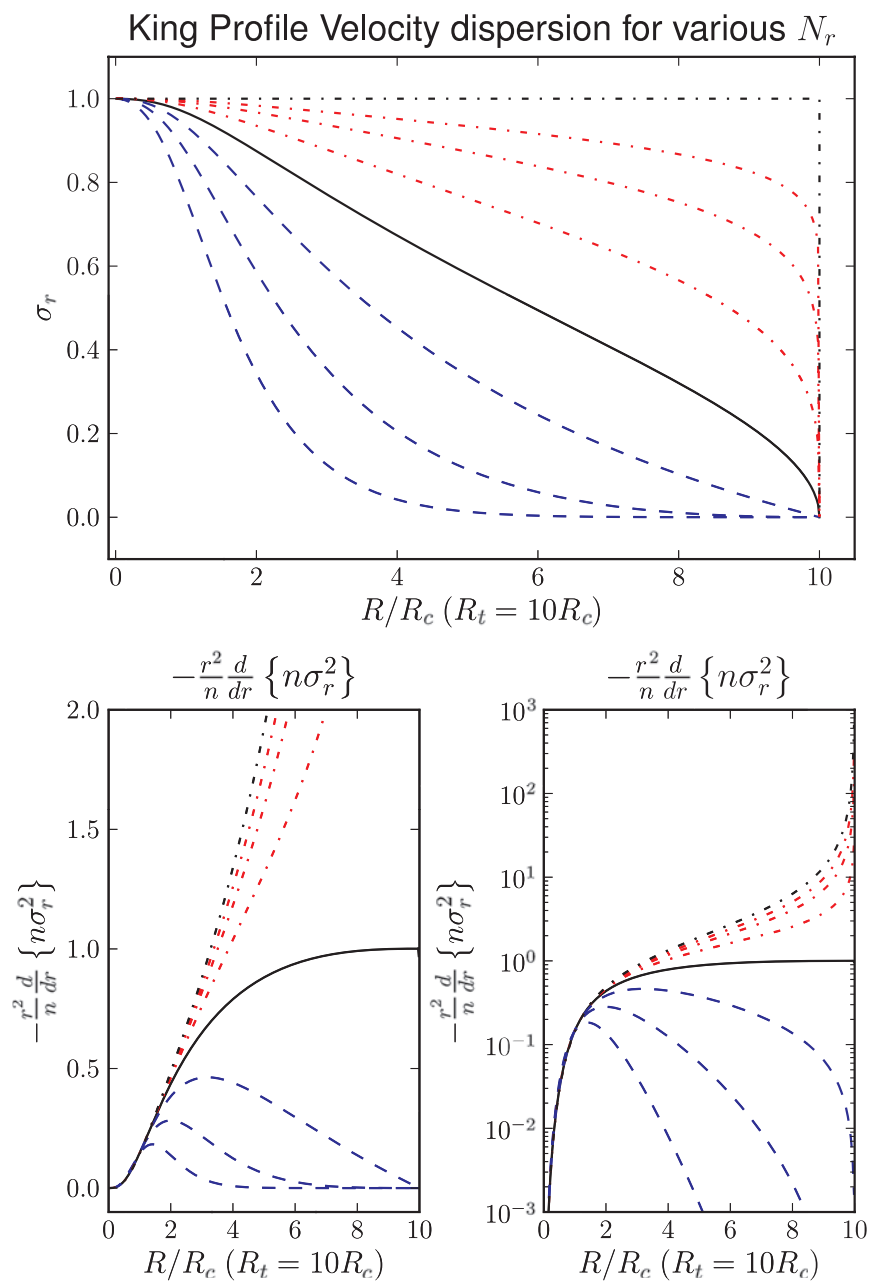

Figure 27. Velocity dispersion profiles following Leonard \& Merritt (1989, LM89). Top panel shows the radial velocity dispersion profile under the same range of velocity anisotropy parameters $N_{r}$ used in Figure 1 of LM89. Dashed lines show (left to right): $N_{r}=8,4,2$. Dot-dashed lines show (left to right): $N_{r}=0.5,0.25,0.125,0.0$. Bottom panels show the behavior of the first term in the Jeans equation under the same values of $N_{r}$, showing divergence for $N_{r}<1$. (A color version of this figure is available in the online journal.)

this gives

$$
\begin{aligned}
u & \equiv 1+\left(r / r_{0}\right)^{2} \\
n(r) & =n_{0} u^{-5 / 2} \\
\sigma_{r}(r) & =\sigma_{0} u^{-N_{r} / 4} \\
\sigma_{t}(r) & =\sigma_{0} u^{-N_{t} / 4} \\
\Sigma(R) & =\Sigma_{0} u^{-2},
\end{aligned}
$$

where $N_{r}, N t$ are positive indices that are used to modify the dispersion profile for velocity anisotropy.

Note that while $\Sigma_{0}=(4 / 3) r_{0} \rho_{0}$ depends on $\left(r_{0}, N r, N t, \sigma_{0}^{2}\right)$, this refers to the surface density by mass, not by the number of stars observed per $\mathrm{pc}^{2}$. The relationship between the surface density (mass) and surface density (number of tracers per $\mathrm{pc}^{2}$ ) depends on the mass function and the depth of observations, and for the purpose of the modeling is treated as a free parameter to be optimized out when evaluating the fit of the model to surface density data.

2. Use the Jeans equation to evaluate the enclosed mass distribution corresponding to the anisotropic velocity dispersions just derived, but assume the mass density of the measured stars (as opposed to the underlying mass distribution) is distributed isotropically as $n(r)$ :

$$
G M(<r)=-\frac{r^{2}}{n} \frac{d}{d r}\left\{n \sigma_{r}^{2}\right\}-2 r\left(\sigma_{r}^{2}-\sigma_{t}^{2}\right),
$$

which for the Plummer model (1) becomes

$$
\begin{aligned}
& M(<r)=\frac{r \sigma_{0}^{2}}{G}\left[\left(\frac{r}{r_{0}}\right)^{2}\left(5+N_{r}\right) u^{-(N r+2) / 2}-2 u^{N_{r} / 2}+2 u^{-N t / 2}\right] \\
& \left(\frac{M(<r)}{M_{\odot}}\right)=231.3 \times\left(\frac{\sigma_{0}}{\mathrm{~km} \mathrm{~s}^{-1}}\right)^{2}\left(\frac{r}{\mathrm{pc}}\right) \cdots \\
& \cdots \times\left[\left(\frac{r}{r_{0}}\right)^{2}\left(5+N_{r}\right) u^{-(N r+2) / 2}-2 u^{N_{r} / 2}+2 u^{-N t / 2}\right] .
\end{aligned}
$$

3. Use the enclosed mass to estimate the volume density profile $\rho(r)$ of the underlying mass distribution:

$$
\rho=\frac{1}{4 \pi r^{2}} \frac{d}{d r}\{M(<r)\},
$$

which for the Plummer model is

$$
\begin{aligned}
\rho(r)= & \frac{\sigma_{0}^{2}}{4 \pi G r_{0}^{2}}\left\{u ^ { - ( N _ { r } + 2 ) / 2 } \left(2 N_{r}+\left(5+N_{r}\right)\right.\right. \\
& \left.\times\left[3-\left(\frac{r}{r_{0}}\right)^{2}\left(\frac{N_{r}+2}{u}\right)\right]\right) \cdots \\
& \cdots-2 N_{t} u^{-\left(N_{t}+2\right) / 2}+2\left(\frac{r_{0}}{r}\right)^{2} \\
& \left.\times\left(u^{-N_{t} / 2}-u^{-N_{r} / 2}\right)\right\} \\
\left(\frac{\rho}{M_{\odot} \mathrm{pc}^{-3}}\right)= & 18.4\left(\frac{\sigma_{0}}{\mathrm{~km} \mathrm{~s}^{-1}}\right)^{2}\left(\frac{r_{0}}{p c}\right)^{-2} \times\{\cdots\}
\end{aligned}
$$

with the lower form giving $\rho(r)$ in units $M_{\odot} \mathrm{pc}^{-3}$ with $r_{0}$ in pc and $\sigma_{0}$ in $\mathrm{km} \mathrm{s}^{-1}$. As $r \rightarrow 0$ then \{\}$\rightarrow 15+5 N_{r}-2 N_{t}$, so the central volume density becomes:

$$
\rho_{0}=\frac{\sigma_{0}^{2}}{4 \pi G r_{0}^{2}} \times\left\{15+5 N_{r}-2 N_{t}\right\} .
$$

4. Evaluate the mass observed within projected radius $R$ onsky to predict $M(R<0.4 \mathrm{pc})$. This is given by

$$
\begin{aligned}
M\left(<R_{f}\right) & =4 \pi \int_{0}^{R_{f}} \int_{R}^{+\infty} \frac{r R \rho(r)}{\sqrt{r^{2}-R^{2}}} d r d R \\
& =\frac{\sigma_{0}^{2}}{G r_{0}^{2}} \int_{0}^{R_{f}} \int_{R}^{+\infty} \frac{r R}{\sqrt{r^{2}-R^{2}}}\{\} d r d R,
\end{aligned}
$$

where the double integral is evaluated numerically.

5. To compare the anisotropic dispersion profile to data, project the model dispersions onto the sky. The projection is the same as for the King model:

$$
\begin{aligned}
\sigma_{R}^{2}(R) & =\frac{\int_{R}^{+\infty} \frac{r n \sigma_{t}^{2}}{\sqrt{r^{2}-R^{2}}}\left[\frac{R^{2}}{r^{2}} \sigma_{r}^{2}+\left(1-\frac{R^{2}}{r^{2}}\right) \sigma_{t}^{2}\right] d r}{\int_{R}^{+\infty} \frac{r n}{\sqrt{r^{2}-R^{2}}} d r} \\
\sigma_{T}^{2}(T) & =\frac{\int_{R}^{+\infty} \frac{r n \sigma_{t}^{2}}{\sqrt{r^{2}-R^{2}}} d r}{\int_{R}^{+\infty} \frac{r n}{\sqrt{r^{2}-R^{2}}} d r}
\end{aligned}
$$

(note that $n(r)$ should be used in this step, not $\rho(r)$ ). 

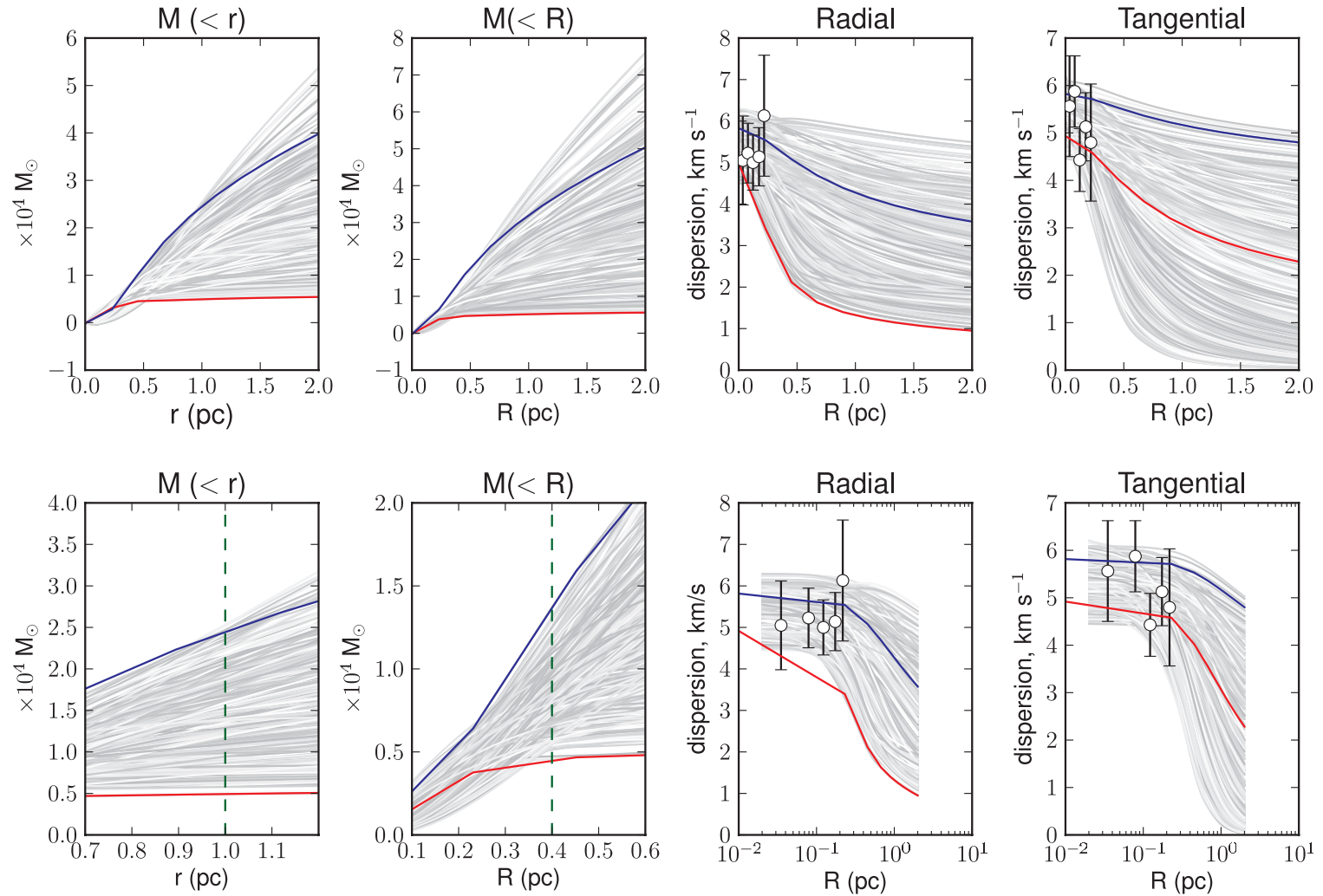

Figure 28. Radial profiles drawn from the 95\% confidence region for Plummer model parameters. Reading left to right: mass within a sphere of radius $r$ pc, projected mass within cylindrical radius $R$ pc, projected-radial and tangential velocity dispersion components. Dashed green lines in the lower left two panels indicate 1.0 and $0.4 \mathrm{pc}$. The solid blue and red curves indicate the upper and lower bounds for $M(R<0.4 \mathrm{pc})$.

(A color version of this figure is available in the online journal.)

\section{REFERENCES}

Allison, R. J., Goodwin, S. P., Parker, R. J., et al. 2009, ApJ, 700, 99 Anderson, J., \& van der Marel, R. P. 2010, ApJ, 710, 1032

Babusiaux, C., Gómez, A., Hill, V., et al. 2010, A\&A, 519, A77

Bartko, H., Martins, F., Trippe, S., et al. 2010, ApJ, 708, 834

Bastian, N., Covey, K. R., \& Meyer, M. R. 2010, ARA\&A, 48, 339

Bastian, N., Gieles, M., Goodwin, S. P., et al. 2008, MNRAS, 389, 323

Benjamin, R. A., Churchwell, E., Babler, B. L., et al. 2005, ApJ, 630, L149

Bevington, P. R., \& Robinson, D. K. (ed.) 2003, Data Reduction and Error Analysis for the Physical Sciences (3rd ed.; Boston, MA: McGraw-Hill)

Bishop, C. M. (ed.) 2006, Pattern Recognition and Machine Learning (1st ed.; New York: Springer)

Bonnell, I. A., \& Rice, W. K. M. 2008, Science, 321, 1060

Bonnell, I. A., Vine, S. G., \& Bate, M. R. 2004, MNRAS, 349, 735

Bovy, J., Hogg, D. W., \& Roweis, S. T. 2011, Ann. Appl. Stat., 5, 1657

Cabrera-Lavers, A., Hammersley, P. L., González-Fernández, C., et al. 2007, A\&A, 465, 825

Chatterjee, S., Goswami, S., Umbreit, S., et al. 2009, arXiv:0911.1483

Clarkson, W., Sahu, K., Anderson, J., et al. 2008, ApJ, 684, 1110

Cotera, A. S., Erickson, E. F., Colgan, S. W. J., et al. 1996, ApJ, 461, 750

Crowther, P. A., Schnurr, O., Hirschi, R., et al. 2010, MNRAS, 408, 731

Dib, S. 2007, J. Korean Astron. Soc., 40, 157

Diolaiti, E., Bendinelli, O., Bonaccini, D., et al. 2000, A\&AS, 147, 335

Dwek, E., Arendt, R. G., Hauser, M. G., et al. 1995, ApJ, 445, 716

Elmegreen, B. G., Klessen, R. S., \& Wilson, C. D. 2008, ApJ, 681, 365

Espinoza, P., Selman, F. J., \& Melnick, J. 2009, A\&A, 501, 563

Figer, D. F. 2005, Nature, 434, 192

Figer, D. F., Kim, S. S., Morris, M., et al. 1999, ApJ, 525, 750

Figer, D. F., Najarro, F., Gilmore, D., et al. 2002, ApJ, 581, 258

Fritz, T., Gillessen, S., Trippe, S., et al. 2010, MNRAS, 401, 1177

Fruchter, A. S., \& Hook, R. N. 2002, PASP, 114, 144

Ghez, A. M., Hornstein, S. D., Lu, J. R., et al. 2005, ApJ, 635, 1087

Ghez, A. M., Salim, S., Weinberg, N. N., et al. 2008, ApJ, 689, 1044

Gieles, M., Sana, H., \& Portegies Zwart, S. F. 2010, MNRAS, 402, 1750

Gillessen, S., Eisenhauer, F., Trippe, S., et al. 2009, ApJ, 692, 1075
Harayama, Y., Eisenhauer, F., \& Martins, F. 2008, ApJ, 675, 1319

Harfst, S., Portegies Zwart, S., \& Stolte, A. 2010, MNRAS, 409, 628

Howard, C. D., Rich, R. M., Clarkson, W., et al. 2009, ApJ, 702, L153

Jones, B. F., \& Walker, M. F. 1988, AJ, 95, 1755

Kim, S. S., Figer, D. F., Kudritzki, R. P., \& Najarro, F. 2006, ApJ, 653, L113

Kim, S. S., Figer, D. F., Lee, H. M., \& Morris, M. 2000, ApJ, 545, 301

Kim, S. S., \& Morris, M. 2003, ApJ, 597, 312

Kim, S. S., Morris, M., \& Lee, H. M. 1999, ApJ, 525, 228

King, I. 1962, AJ, 67, 471

Klessen, R. S., Spaans, M., \& Jappsen, A.-K. 2007, MNRAS, 374, L29

Kozhurina-Platais, V., Girard, T. M., Platais, I., et al. 1995, AJ, 109, 672

Kroupa, P. 2002, Science, 295, 82

Krumholz, M. R., \& McKee, C. F. 2008, Nature, 451, 1082

Kuijken, K., \& Rich, R. M. 2002, AJ, 124, 2054

Lampton, M., Margon, B., \& Bowyer, S. 1976, ApJ, 208, 177

Lang, C. C. 2004, in ASP Conf. Ser. 322, The Formation and Evolution of Massive Young Star Clusters, ed. H. J. G. L. M. Lamers, L. J. Smith, \& A. Nota (San Francisco, CA: ASP), 179

Launhardt, R., Zylka, R., \& Mezger, P. G. 2002, A\&A, 384, 112

Lecureur, A., Hill, V., Zoccali, M., et al. 2007, A\&A, 465, 799

Lejeune, T., \& Schaerer, D. 2001, A\&A, 366, 538

Leonard, P. J. T., \& Merritt, D. 1989, ApJ, 339, 195

Leonard, P. J. T., Richer, H. B., \& Fahlman, G. G. 1992, AJ, 104, 2104

Lu, J. R., Ghez, A. M., Hornstein, S. D., et al. 2009, ApJ, 690, 1463

Martins, F., Hillier, D. J., Paumard, T., et al. 2008, A\&A, 478, 219

McCrady, N., Lu, J., Clarkson, W., et al. 2011, in ASP Conf. Ser. 439, The Galactic Center: A Window to the Nuclear Environment of Disk Galaxies, ed. M. R. Morris, Q. D. Wang, \& F. Yuan (San Francisco, CA: ASP), 121

McKee, C. F., \& Ostriker, E. C. 2007, ARA\&A, 45, 565

McWilliam, A., \& Rich, R. M. 1994, ApJS, 91, 749

McWilliam, A., \& Zoccali, M. 2010, ApJ, 724, 1491

Miller, G. E., \& Scalo, J. M. 1979, ApJS, 41, 513

Morris, M. 1993, ApJ, 408, 496

Morris, M., \& Yusef-Zadeh, F. 1989, ApJ, 343, 703

Nagata, T., Woodward, C. E., Shure, M., \& Kobayashi, N. 1995, AJ, 109, 167 Najarro, F., Figer, D. F., Hillier, D. J., \& Kudritzki, R. P. 2004, ApJ, 611, L105 
Picaud, S., \& Robin, A. C. 2004, A\&A, 428, 891

Platais, I., Kozhurina-Platais, V., Mathieu, R. D., Girard, T. M., \& van Altena, W. F. 2003, AJ, 126, 2922

Portegies Zwart, S., Gaburov, E., Chen, H.-C., \& Guerkan, M. A. 2007, MNRAS, 378, L29

Portegies Zwart, S. F., Makino, J., McMillan, S. L. W., \& Hut, P. 2002, ApJ, 565,265

Portegies Zwart, S. F., McMillan, S. L. W., \& Gieles, M. 2010, ARA\&A, 48, 431

Press, W. H., Teukolsky, S. A., Vetterling, W. T., \& Flannery, B. P. 2002, Numerical Recipes in C++: The Art of Scientific Computing (2nd ed.; Cambridge: Cambridge Univ. Press)

Robin, A. C., Marshall, D. J., Reylé, C., \& Schultheis, M. 2009, in SF2A2009: Proc. Annual Meeting of the French Society of Astronomy and Astrophysics, ed. M. Heydari-Malayeri, C. Reylé, \& R. Samadi, published online at http://sf2a.cesr.fr/2009/proceedings2009.html, 221

Salpeter, E. E. 1955, ApJ, 121, 161
Sanders, W. L. 1971, A\&A, 14, 226

Schödel, R., Merritt, D., \& Eckart, A. 2009, A\&A, 502, 91

Serabyn, E., Shupe, D., \& Figer, D. F. 1998, Nature, 394, 448

Shen, J., Rich, R. M., Kormendy, J., et al. 2010, ApJ, 720, L72

Soto, M., Rich, R. M., \& Kuijken, K. 2007, ApJ, 665, L31

Stolte, A., Brandner, W., Grebel, E. K., Lenzen, R., \& Lagrange, A.-M. 2005, ApJ, 628, L113

Stolte, A., Ghez, A. M., Morris, M., et al. 2008, ApJ, 675, 1278

Stolte, A., Grebel, E. K., Brandner, W., \& Figer, D. F. 2002, A\&A, 394, 459

Stolte, A., Morris, M. R., Ghez, A. M., et al. 2010, ApJ, 718, 810

van Dam, M. A., Bouchez, A. H., Le Mignant, D., et al. 2006, PASP, 118, 310

Wizinowich, P. L., Le Mignant, D., Bouchez, A. H., et al. 2006, PASP, 118 , 297

Yelda, S., Lu, J. R., Ghez, A. M., et al. 2010, ApJ, 725, 331 (Y10)

Yusef-Zadeh, F., Law, C., Wardle, M., et al. 2002, ApJ, 570, 665

Yusef-Zadeh, F., Morris, M., \& Chance, D. 1984, Nature, 310, 557

Zhao, H., Spergel, D. N., \& Rich, R. M. 1994, AJ, 108, 2154 


\title{
ERRATUM: "PROPER MOTIONS OF THE ARCHES CLUSTER WITH KECK LGS-ADAPTIVE OPTICS: THE FIRST KINEMATIC MASS MEASUREMENT OF THE ARCHES (2012, ApJ, 751, 132)
}

\author{
W. I. Clarkson ${ }^{1,2}$, A. M. Ghez ${ }^{1}$, M. R. Morris ${ }^{1}$, J. R. Lu $^{3,4}$, A. Stolte ${ }^{5}$, N. McCrady ${ }^{6}$, T. Do ${ }^{7}$, And S. Yelda ${ }^{1}$ \\ ${ }^{1}$ Division of Astronomy \& Astrophysics, University of California, Los Angeles, Physics and Astronomy Building, \\ 430 Portola Plaza, Box 951547, Los Angeles, CA 90095-1547, USA \\ 2 Department of Astronomy, Indiana University, Bloomington, 727 East 3rd Street, Swain West 319, Bloomington, IN 47405-7105, USA; wiclarks@indiana.edu \\ ${ }^{3}$ Institute for Astronomy, 2680 Woodlawn Drive, Honolulu, HI 96822-1839, USA \\ ${ }^{4}$ Department of Astronomy, California Institute of Technology, 1200 East California Blvd, Pasadena CA 91125, USA \\ 5 Argelander Institut für Astronomie, Universität Bonn, Auf dem Hügel 71, 53121 Bonn, Germany \\ ${ }^{6}$ Department of Physics and Astronomy, University of Montana, 32 Campus Drive, No. 1080, Missoula, MT 59812, USA \\ ${ }^{7}$ Department of Physics \& Astronomy, University of California, Irvine, 4129 Frederick Reines Hall, Irvine, CA 92697-4575, USA \\ Received 2012 May 22; published 2012 June 15
}

Due to an error at the publisher, the affiliations of the authors were listed incorrectly. The corrected version is listed above. IOP Publishing sincerely regrets this error. 